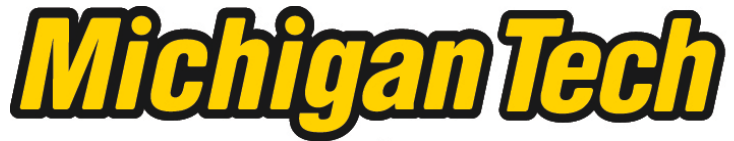 \\ Michigan Technological University Create the Future Digital Commons @ Michigan Tech
}

Reaction Control in Quiescent Systems of Free-Radical Retrograde-Precipitation Polymerization

Vijaya Raghavan Tirumala

Michigan Technological University

Follow this and additional works at: https://digitalcommons.mtu.edu/etds

Part of the Chemical Engineering Commons, and the Materials Science and Engineering Commons Copyright 2003 Vijaya Raghavan Tirumala

\section{Recommended Citation}

Tirumala, Vijaya Raghavan, "Reaction Control in Quiescent Systems of Free-Radical RetrogradePrecipitation Polymerization", Dissertation, Michigan Technological University, 2003.

https://doi.org/10.37099/mtu.dc.etds/733

Follow this and additional works at: https://digitalcommons.mtu.edu/etds

Part of the Chemical Engineering Commons, and the Materials Science and Engineering Commons 


\title{
REACTION CONTROL IN QUIESCENT SYSTEMS OF FREE-RADICAL RETROGRADE-PRECIPITATION

\author{
POLYMERIZATION
}

By

VIJAYA RAGHAVAN TIRUMALA

\author{
A DISSERTATION \\ Submitted in partial fulfillment of the requirements for the degree of \\ DOCTOR OF PHILOSOPHY \\ (Chemical Engineering) \\ MICHIGAN TECHNOLOGICAL UNIVERSITY \\ 2003 \\ (C) 2003 V. R. Tirumala
}


This dissertation, "Reaction Control in Quiescent Systems of Free-Radical

Retrograde-Precipitation Polymerization", is hereby approved in partial fulfillment of the requirements for the degree of DOCTOR OF PHILOSOPHY in the field of Chemical Engineering.

Department of Chemical Engineering

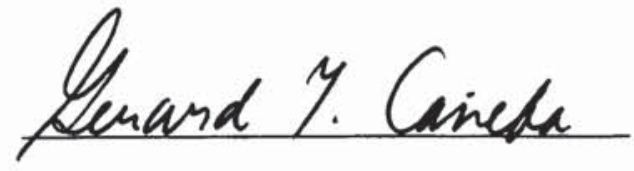

(Dr. Gerard T. Caneba)

Advisor

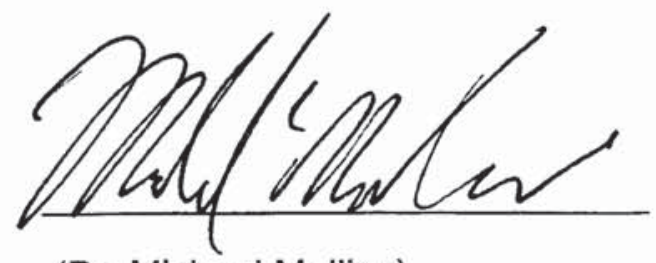

(Dr. Michael Mullins)

Department Chair

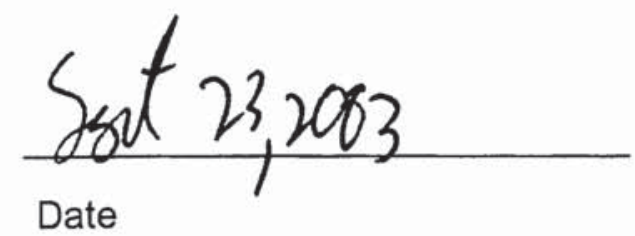


To Ammamma and Tataji: For your ever lasting love... 


\section{Acknowledgements}

It was a great privilege to work with Dr. Gerard Caneba, who let me hop onto his ongoing train of research. That sure was an adventurous journey for us! For the past four years, Dr. Caneba has been more a friend than an advisor. His continuous support, enthusiasm and encouragement served as the guiding lights of this work. I am thankful to Drs. Nam Kim, Joseph Holles and Douglas Swenson for serving on my dissertation committee, and for reserving their best for my final defense. I also would like to thank my colleagues and friends Jayashankar Yaddanapudi, Yi Zhao, and Shaolong Qu for keeping me in good spirits whenever the chips are down. Dr. Yadunandan Dar was ever ready to offer me his tips and advices and also his insights into the process in study. His free flowing discussions made several of my concepts clear and have certainly helped in the progress of my research.

Working at the Advanced Photon Source of the Argonne National Laboratories was a truly rewarding experience in every sense of the word. Dr. Derrick Mancini stands out for being a perfect mentor: giving me a free hand in research while making sure that $\mathrm{I}$ do not stray away from the chosen path. But for his financial support, this project would not have made as much progress as it. did. I am also grateful to Dr. Ralu Divan and Judi Yaeger of the microlithography group at the APS, for their excellent lab support during the course of my work. Dr. Mike Moldovan and Dr. Ralu Divan also taught me the fundamentals of lithography during the initial stage of this project. Dr. Francesco DeCarlo and Dr. Yong Chu of the 2-BM beamline were always there whenever help was 
needed. Their support in the timely maintenance of the beamline and in scheduling my experiments is sincerely appreciated. Dr. Hsien-Hau Wang's group in the Materials Sciences Division helped me start at Argonne, without which none of this work would have been possible. I also would like to thank his post doctoral researchers Dr. Catherine Han and Dr. Gerald Willing for being such good friends and for helping me out whenever in need. Dr. Thiyagarajan and his group helped me learn the principles of small-angle scattering. I would especially like to thank Dr. Jaby Jacob for clearing my innumerable doubts when I was just starting out on SAS. Dr. Liang Guo and Denis Wozniak did a great job in maintaining the small angle neutron diffractometer during the course of my experiments on gels. Denis was a gem of a person and I cherish our friendship for the years to come.

Working on my degree over a long distance involved many hassles that required paperwork. I am immensely grateful to Alexis for making my job easier by checking with me from time to time and for keeping tabs on my lapses, if any. Similar help from Barbara, Janet and Rachel at the APS made dealing with all paperwork a pleasure. I am also thankful to Susan P., the resident editor at the APS, for patiently going through several versions of my works to iron out all the irregularities in my writing style.

Javier and Chris at MTU were wonderful while fixing problems related to Windows computing environment. Mac support group over at APS were also marvelous in their support to my Macintosh environment, although most of the problems concerned Microsoft products. Rodney, Jon and Alessandra were always thought provoking on the issues of American psyche and cross-cultural similarities and differences, my favorite topic of discussion. 
Over the years I made many great friends both personally and professionally, many of whom I hope to keep in touch with. I thank Dr. Anil Kandalam and Dr. Raghava Panguluri for constantly encouraging me with great feedback. I am sure they would be happy to see me graduate. I also thank my friends Jaya, Prasad, Jinesh, Raj, Rajesh, Jitendra, Rama, and Srinivas for making my stay at MTU a memorable one. I also would like to thank Ram Kuchibhotla for all his help during my stay in Illinois. Last, but not the least, I would like to thank my dear friends Mahesh, Veena and Koti for always being there for me and for their fantastic support. Thank you guys!!!

Above all, I am grateful forever to my grand parents and parents for giving me a good life and for shaping me into what I am today. I am thankful to my maternal uncles as well for their continuous support through all my pressures and pains and for enjoying my progress as their pleasure. I also would like to acknowledge our Bobbili Mama's support and the inspirational role he played in my life. Finally, I thank THE ONE for giving me enough chances to correct my mistakes and for making my life an eventful ride so far.

Vijaya Raghavan Tirumala 09/22/03 
Abstract

\section{Abstract}

Reaction control is of utmost importance in complex chemistries: especially in polymerization reactions, which are notorious for their "run away" periods during which the exothermic chain reaction is hard to contain. The understanding of any new polymerization process is, therefore, not complete without a deep insight into the controlling characteristics of the reaction.

Free-radical retrograde-precipitation polymerization, FRRPP in short, is a novel polymerization process discovered by Dr. Gerard Caneba in the late 1980 s. The focus during the early exploration of FRRPP was more on understanding the mechanistic behavior and the innovative possibilities this discovery had to offer. Unlike conventional precipitation processes that occur below the upper critical solution temperature, FRRPP, occurring in an inverse precipitation region, uses the fine interplay between the exothermic reaction and the phase (in)equilibrium to exert control over polymer chain growth, resulting in trapped radicals as the reaction proceeds. This unique behavior was demonstrated by past researchers using both experimental and theoretical means. The current study is aimed at gaining a better understanding of the reaction mechanism of the FRRPP and its thermodynamically-driven features that are predominant in controlling the chain reaction. 
A previously developed mathematical model to represent free radical polymerization kinetics was used to simulate a classic bulk polymerization system from the literature. Unlike other existing models, such a sparse-matrix-based representation allows one to explicitly accommodate the chain length dependent kinetic parameters. At the outset, this approach was shown to accurately simulate the bulk polymerization of methyl methacrylate although it falls short of describing the kinetics of polymer chains with a chain length greater than 2000. However, the model can still be used to simulate controlled polymerization systems such as FRRPP or dead-end polymerization process, where the maximum chain length is inherently limited.

Extrapolating from the past results, mixing was experimentally shown to be exerting a significant influence on reaction control in FRRPP systems. Mixing alone drives the otherwise severely diffusion-controlled reaction propagation in phase-separated polymer domains. Therefore, in a quiescent system, in the absence of mixing, it is possible to retard the growth of phase-separated domains, thus producing isolated polymer nanoparticles (globules). Such a diffusion-controlled, self-limiting phenomenon of chain growth was also observed using time-resolved small angle $\mathrm{x}$-ray scattering studies of reaction kinetics in quiescent systems of FRRPP. The method was then extended to the synthesis of polymeric nanoparticles from a monomer that polymerizes into a thermorevèrsible hydrogel. The mechanistic understanding derived from these experimental results led to a novel radiation induced synthesis of hydrogels that can respond to a variety of external stimuli. 
Combining the concept of self-limiting chain growth in quiescent FRRPP systems with spatioselective reaction initiation of lithography, microgel structures were synthesized in a single step, without the use of molds or additives. Hard x-rays from the bending magnet radiation of a synchrotron were used as an initiation source, instead of the more statistally-oriented chemical initiators. Such a spatially-defined reaction was shown to be self-limiting to the irradiated regions following a polymerization-induced self-assembly phenomenon. The pattern transfer aspects of this technique were, therefore, studied in the FRRP polymerization of $\mathrm{N}$-isopropylacrylamide (NIPAm) and methacrylic acid (MAA), a thermoreversible and ionic hydrogel, respectively. Reaction temperature increases the contrast between the exposed and unexposed zones of the formed microgels, while the irradiation dose is directly proportional to the extent of phase separation. The response of Poly (NIPAm) microgels prepared from the technique described in this study was also characterized by small angle neutron scattering. It was observed that an increase in absorbed dose increases the response rates of the gels, which indirectly implies an increase in crosslink density. Gels prepared at $20 \%$ monomer concentration reach a critical crosslinking density beyond which the gel collapse is minimized.

The current approach was then applied to the synthesis of high aspect-ratio (height-towidth ratio) microgel structures, and discussed from an application perspective. The experimental setup used in all the exposures was also modeled to calculate parameters like dose absorption profiles of the reactive mixtures and total absorbed dose. The microgel structures synthesized in this study can be potentially used in a wide range of applications in controlled release and other soft matter based MEMS components such as microfluidic flow valves, size-based nanoseparation systems etc. 


\section{TABLE OF CONTENTS}

TITLE

PAGE NO.

ABSTRACT i

TABLE OF CONTENTS iv

LIST OF FIGURES Ix ix

LIST OF TABLES X xii

1. Reaction Control in the FRRPP Process: A Synopsis 1

Introduction 1

Modeling and Simulation of Free Radical Polymerizations 2

Mixing Effects in Quiescent FRRPP Systems 3

Spatially Controlled In Situ Synthesis of Polymers 6

$\begin{array}{ll}\text { Synthesis of Nanocomposite Microgels } & 10\end{array}$

References 11

2. A Novel Sparse Matrix Representation of FRP Simulations 13

Introduction to Free-Radical Polymerization Simulations $\quad 13$

$\begin{array}{ll}\text { Free Radical Polymerization Kinetics } & 15\end{array}$

$\begin{array}{ll}\text { Modeling Free Radical Kinetics } & 17\end{array}$ 
A Novel Sparse Matrix Representation 20

Results and Discussion 23

$\begin{array}{ll}\text { Conclusions } & 31\end{array}$

References 33

3. Nanoparticles from Quiescent FRRPP Systems 35

Introduction to Polymeric Nanostructures 35

Experimental 39

Materials Used $\quad 39$

Polymerization in a Stirred-Tank Reactor $\quad 39$

Polymerization in a Quiescent-Fluid Reactor $\quad 40$

Sample Preparation for AFM Analysis $\quad 41$

In Situ SAXS Study of Methacrylic Acid Polymerization 41

Growing Polymer Chain Behavior in a Poor Solvent $\quad 42$

Results and Discussion $\quad 44$

$\begin{array}{ll}\text { Conclusions } & 52\end{array}$

References $\quad 54$

4. A Novel Strategy for the Synthesis of Poly (NIPAm) Microstructures $\mathbf{5 8}$

Review of Radiation Induced Polymerization $\quad 58$

Gamma-ray Polymerization of Styrene/Acrylates 61 
Introduction to Hydrogels

Experimental

Polymerization in a Quiescent-Fluid Reactor

Synchrotron-Radiation-Induced Polymerization

Results and Discussion

Conclusions

References

5. A Novel Spatially-Controlled Synthesis of Microgels

Introduction to Radiation-Induced Materials Synthesis

Polymerization in Inverse Precipitation Systems

Experimental

Materials Used

Sample Preparation

Spatially-Controlled Polymerization

Microgel Characterization

Results and Discussion

Polymerization Induced Self-Assembly

Synthesis of Ionic Microgels 
6. Morphological Study of PNIPAm Gels Using SANS

Introduction to Small Angle Scattering Study of Hydrogels 95

$\begin{array}{ll}\text { Experimental } & 98\end{array}$

$\begin{array}{ll}\text { Materials Used } & 98\end{array}$

Synchrotron-Radiation-Induced Polymerization $\quad 98$

Small Angle Neutron Scattering $\quad 99$

$\begin{array}{ll}\text { Results and Discussion } & 101\end{array}$

$\begin{array}{ll}\text { Initial Morphology } & 101\end{array}$

$\begin{array}{ll}\text { Data Reduction } & 102\end{array}$

$\begin{array}{ll}\text { Change in Morphology } & 103\end{array}$

Influence of Monomer Concentration $\quad 105$

$\begin{array}{ll}\text { Conclusions } & 107\end{array}$

$\begin{array}{ll}\text { References } & 109\end{array}$

7. Lithographically Assisted Synthesis of Hydrogel Microstructures 111

$\begin{array}{ll}\text { Introduction } & 111\end{array}$

$\begin{array}{ll}\text { Exposure Station Configuration } & 112\end{array}$ 
High Aspect Ratio Hydrogel Microstructures

Discussion

Conclusions

References

8. Conclusions and Future Directions

121

Conclusions

121

Future Directions

122

Folding Kinetics of a Polymerizing Chain in a Poor

Solvent

Synthesis of Nanocomposite Nanoparticles

Acknowledgements

APPENDIX I MATLAB Code for FRP Simulations

125

APPENDIX II The Advanced Photon Source: A Primer

130

APPENDIX III Beamline Configuration: 2-BM

133 


\section{LIST OF FIGURES}

Figure 2.1 Conversion and kinetic rate coefficients vs. time

(comparison between experimental and simulation

results).

Figure 2.2 Polymer population evolution in time 26

$\begin{array}{lll}\text { Figure 2.3 Evolution of dead polymer chains with time } & 27\end{array}$

Figure 2.4 Evolution of polymer radical population with time 28

Figure 2.5. Radical concentration profile as a function of scaled 29

time and chain length.

Figure 2.6 Maximum allowable chain length vs. computation time 31

Figure 3.1 Binary phase diagram of a polymer-solvent system in 36 temperature.

Figure 3.2 Reaction trajectory through a ternary phase envelope. 43

Figure 3.3 Atomic Force Microscopic scan of product from a well- 45 mixed polymerization of styrene in ether.

Figure 3.4 Nanoparticles from a quiescent polymerizing system 46 of styrene/EGDMA/ether.

Figure 3.5 AFM image of nanoparticles from styrene/EGDMA polymerized in a mixed solvent of (t-BA-water).

Figure 3.6 AFM image of nanoparticles from styrene/ether system, 
without the crosslinker.

Figure 3.7 Time-resolved small angle scattering profile of MAA polymerization in water at $60^{\circ} \mathrm{C}$.

Figure 3.8 Time-resolved small angle scattering profiles of MAA polymerization at $80^{\circ} \mathrm{C}$.

Figure 3.9 Kinetics of polymer chain collapse at $60^{\circ} \mathrm{C}$

Figure 4.1 Schematic of the experimental set-up in x-rays induced 69 spatially-controlled polymerization.

Figure 4.2 Nanogel particles based on N-isopropylacrylamide (NIPAm) 70

Figure 4.3. Optical micrograph of a Poly (NIPAm) pattern and its profile 71

Figure 5.1 Schematic of the reactive fluid containment system and the $\quad 81$ experimental set-up at the beamline 2-BM-B.

Figure 5.2 Polymerization-Induced Self-Assembly. 83

Figure 5.3 Thermoreversible microgels of different shapes and sizes. $\quad 85$

Figure 5.4 Influence of process parameters on the pattern transfer aspects 87 of ionic microgels.

Figure 5.5 Poly (NIPAm-co-Au) nanohybrid microgels 89

Figure 5.6 Graft copolymer microgels based on Poly (NIPAm-g-PEG600) 90

Figure 6.1 Power spectrum of the APS bending magnet radiation and 98 the attenuated profiles past monomer solutions of a certain composition and thickness. 
Figure 6.2 Small angle neutron scattering profiles of PNIPAm gels

prepared from synchrotron radiation induced polymerization.

Figure 6.3 Temperature-dependent swelling characteristics of PNIPAm

103

prepared from SRIP.

Figure 6.4 Effect of absorbed dose of the morphological transitions of

PNIPAm gels.

Figure 6.5 Effect of monomer concentration on the morphological response of PNIPAm gels.

Figure 7.1 Power spectrum of the bending magnet radiation at the

2-BM-B beamline of the Advanced Photon Source

Figure 7.2 Process methodology of the lithographically-assisted

synthesis of polymers.

Figure 7.3 Dose absorption profile in a $500 \mu$-thick $20 \%$ NIPAm solution.

Figure 7.4 Dose absorption profile in a $500 \mu$-thick, $20 \%$ MAA solution.

Figure 7.5 High aspect ratio hydrogel microstructures based on NIPAm. 116

Figure 7.6 Pattern replication aspects of a thermoreversible and an ionic hydrogel.

Figure 7.7 High aspect ratio ionic hydrogel microstructures based on MAA. 118 


\section{LIST OF TABLES}

Table 2.1 Dimensions of matrices used in the free radical polymerization simulations.

Table 2.2 Physical parameters used for the simulation of methyl

methacrylate bulk polymerization

Table 2.3 Kinetic parameters used in the simulation of methyl

methacrylate bulk polymerization.

Table 3.1 Particle analysis of the nanoparticles obtained from the quiescent fluid polymerization of styrene/EGDMA in ether

Table 3.2 Radii of gyration values calculated from the Debye function

for a flexible Gaussian chain, for different FRRPP systems.

Table 7.1 Samples used in small angle scattering measurements 


\section{Chapter 1}

\section{Reaction Control in the FRRPP Process}

\section{Introduction}

Free-radical retrograde precipitation polymerization, or FRRPP for short, was discovered by Dr. Gerard Caneba in the late 1980 s. $^{1}$ A variation of conventional precipitation polymerization, FRRPP occurs in the inverse phase envelope of a polymersolvent system, also called the lower critical solution temperature (LCST). Relying on a polymer-solvent phase inequilibrium that is directly proportional to temperature, this variation in conventional precipitation polymerization offers better control over the otherwise "run away" polymerization reactions. Past research by Caneba and his coworkers $^{2-5}$ has provided valuable experimental insight into the mechanistic complexities of FRRPP, some of which are still not completely understood. A recent study in FRRPP was directed towards providing a fundamental insight into various aspects of the process including mathematical modeling and simulation. ${ }^{6}$ The simulation failed to accurately represent the process due to lack of theoretical understanding into 
phase equilibrium thermodynamics of a polymer chain in a poor solvent. Nevertheless, given the limited understanding of polymer physics in a poor solvent, ${ }^{*}$ the effort was very well directed and simulation results qualitatively agreed with the kinetics even if not completely justified from a polymer physics perspective.

\section{Modeling and Simulation of Free Radical Polymerizations}

Development of a new model for FRRPP presented a unique challenge that requires a separate and detailed study with considerable effort spent towards studying the kinetics of polymer chain collapse in a poor solvent. Initial efforts in that direction required the modification and implementation of an equation-based modeling approach developed earlier to simulate free-radical polymerization reactions [Chapter 2]. The model was validated by classic bulk polymerization simulations of experimental results from literature. The model developed was quite simple and can simulate the kinetics of the system under study, over the entire range of conversion. The amount of memory required to simulate the entire spectrum of molecular weight distribution limits this approach to systems in which the inherent chain lengths are much smaller than in bulk polymerization systemss. ${ }^{1,78}$ The approach still had to be validated against a case in which the chain lengths are much higher than allowed in the simulation, to completely understand the limitations and shortcomings of this procedure. The model was therefore applied to a classic case of bulk polymerization of methyl methacrylate. ${ }^{9-12}$ It was shown that the

\footnotetext{
* A solvent in which the polymer is insoluble below or above a critical solution temperature is called a poor solvent. Behavior of a growing polymer chain in a poor solvent is explained in detail in Chapter 3.
} 
model, despite its computational limitations, simulates the kinetics of the reactive system well.

The unique feature of this novel approach is that one can a priori simulate the molecular weight distributions from a polymerization reaction. Now that the method has been successfully established, interested researchers can apply this method in the future towards the simulation of controlled polymerization chemistries like dead-end polymerization, FRRPP, ionic polymerization etc. The simulation of precipitation polymerization in a retrograde phase envelope without any adjustable parameters still remains unsolved, unless a conceptual understanding of the polymer physics in a poor solvent is developed. That problem, however, is quite complex in itself and deserves an independent and detailed experimental and theoretical investigation.

\section{Mixing Effects in Quiescent FRRPP Systems}

Extending the experimental and theoretical understanding of past researchers, ${ }^{3,6}$ the current study explored the reaction control aspects of FRRPP from a micro- to nanoscale level. Since the phase inequilibrium in FRRPP systems is caused by non-isothermality, mixing was expected to play a crucial role in reaction control and in preserving live radicals. This hypothesis was experimentally verified in a well-mixed stirred tank reactor and contrasted with similar experiments in a quiescent reactor system [Chapter 3]. In a well-mixed reactor equipped with a stirrer, mixing was shown to be driving and promoting agglomeration of growing polymer chains.

According to the proposed mechanism and past experimental evidence, it is expected that the polymer chains grow to a limiting chain length whereupon they precipitate by 
excluding the previously absorbed solvent. Propagation rates in precipitates existing in a tightly coiled globular state will, therefore, be severely retarded due to the unavailability of monomer within the globule. Although a negative concentration gradient helps the monomer diffusion into the polymer precipitate, the "hot spots" existing within the globule due to the exothermic chain reaction present an uphill diffusion barrier that isolates the growing polymer chain end from the ambient monomer. The unavailability of monomer also decreases the termination reaction rates within the globular precipitates.

This phenomenon is quite similar to that of the "dead-end" polymerization effect observed in bulk polymerization when fast initiators were used at high temperatures. ${ }^{7,8}$ That effect was attributed to the fast depletion of initiator and higher initial reaction rates. The limiting conversions observed in such systems were explained to be a result of the unavailability of initiator (or catalyst, as it was called) and the diffusion-controlled reaction. Once the initiator is completely depleted, the mobility of growing polymer chains is affected by the increased viscosity of the medium, which severely reduces the termination rate constant. The propagation rate coefficient, dependent only on the mobility of primary radicals, remains unaffected by the change in viscosity of the medium. An uncontrolled gel-effect still occurs even if the reaction temperature is only slightly lower than that of dead-end polymerization conditions. ${ }^{8}$ Increase in temperature alone need not be a prerequisite to exert control over the uncontrolled propagation and termination reactions. If the reaction environment is cleverly modified to decrease the propagation and termination rates at higher conversions, one should also be able to achieve limiting conversions even at lower temperatures. This is specifically the case with FRRPP systems, which use the "poor" solvent environment to control the exothermic chain reaction by reactive precipitation. The unavailability of monomer 
within the collapsed globules also results in trapped radical chain ends, which can be preserved until they are exposed to more monomer dissolved in a better solvent environment.

In a well-mixed system, the collapsed globules will once again be physically exposed to more monomer from the solution and exist in a partly solvated state, further driving the reaction. The addition of more monomer units to the solvated polymer chain, on the contrary, increasingly favors the collapse of the growing chain and exclusion of absorbed solvent. Further growth would only be possible by agglomeration, which also is promoted by mixing. Therefore, in a well-mixed reactor, it will be difficult to isolate the growing domains and observe nanoparticles (oligomeric and low chain-end polymer precipitates). Most of the precipitants readily agglomerate forming larger domains. In an unmixed system, polymer precipitants ("hot spots") have to grow in isolation since there will be no direct interaction between them. In view of the highly suppressed propagation rates, growth of isolated globules will be limited, due to the unavailability of monomer. Thus, when a monomer is polymerized in a quiescent and retrograde-precipitation environment, polymeric nanoparticles can be obtained [Chapter 3]. The postfacto atomic force microscopy (AFM) observations verifying the proposed reaction mechanism were also well supported by the in situ time-resolved small-angle scattering measurements.

From the direct synthesis of nanoparticles and the small-angle scattering investigations, it was coneluded that the size of polymer precipitates in quiescent FRRPP systems can be controlled to within a few tens of nanometers. Analogously, when the reaction is spatioselectively initiated with high-energy photons in the absence of chemical initiators, the decrease in propagation and termination reactions induced by chain collapse should 
limit the reaction spread only to the irradiated zones. Thus, in principle, it should be possible to synthesize spatially defined polymer microstructures in an in situ manner.

\section{Spatially Controlled In Situ Synthesis of Polymers}

A feasibility study of this prospect was first conducted as a proof-of-concept [Chapter 4], after which the process was investigated in detail. Poly ( $\mathrm{N}$-isopropylacrylamide) (PNIPAm), which has an LCST at $32^{\circ} \mathrm{C}$, was used as a test monomer in this study. X-rays from a bending magnet radiation of the Advanced Photon Source (APS) were used as an initiation source. ${ }^{\dagger}$ The energy of the x-rays and the power delivered to the sample can be easily varied by changing the optical elements in the path of the beam. Initiation can be spatially defined by exposing the monomer mixture through an $\mathrm{x}$-ray mask fabricated via photolithography and gold electroplating. Radiation, in the form of gamma rays and electron beam, is conventionally used in synthesis and modification of polymers in bulk. Since synchrotron sources were not used for polymer synthesis before, gamma-ray irradiation of monomers and its effects on various polymerizing systems are also briefly reviewed in Chapter 4. High energy radiation is energetic enough to create free radicals from most of the organic molecules, especially at high temperatures. ${ }^{13}$ Hard $\mathrm{x}$-rays from a synchrotron were particularly chosen because of the relative ease of material handling; the task is much harder when initiating with other sources. ${ }^{\ddagger}$ Since propagation is inherently controlled by the phase equilibrium thermodynamics, the

\footnotetext{
† Synchrotron radiation and its characteristics are briefly explained in Appendix II.

¥ Both soft $\mathrm{x}$-rays and electron-beam initiation require exposing the liquid monomer mixture in vacuum.
} 
spatial control exerted by this process should be independent of the method of initiation. This is also supported by the fact that the interaction of $\mathrm{x}$-rays and electron beam with matter is similar, albeit at different energies. The success of this process, therefore, can also be translated to initiation with other sources.

$\mathrm{N}$-isopropylacrylamide (NIPAm), chosen as a model monomer for this study, is well known for its thermoreversible behavior, applied in the areas of controlled release, therapeutics and other bio-related applications. Belonging to the class of hydrogels (hydrophilic polymers that respond to external stimuli), Poly (NIPAm) (PNIPAm) can also be used as temperature sensors, microfluidic valves, etc. The response rates of PNIPAm to an external stimulus are, therefore, of utmost importance in all these applications and depend on the synthesis conditions. ${ }^{14-16}$ Conventional synthesis procedures, which typically use chemical-initiation methods for gel synthesis, produce gels that are slow in their response to an external trigger. This is because such gels are hugely nanoporous which decrease the mobility of water through the gel structure. ${ }^{17}$ Many previously suggested alternative routes to the synthesis of fast-response gels include polymerizing the monomer deeper in its phase envelope (above the LCST, or way below the upper critical solution temperature (UCST) and even mixed exposure to LCST and UCST). Nevertheless, these gels can only exist in bulk without any conformation to a form or size.

A novel-strategy for gel synthesis that not only produces fast-response gels but also makes them conform to any shape or size will be widely useful for a variety of applications. Beebe and coworkers partially achieved that objective by combining photolithography and photopolymerization for gel synthesis, and used the gels as thermoresponsive microfluidic valves. ${ }^{18}$ The approach, nevertheless, is limited in its use 
due to the limitations arising from ultraviolet radiation based photolithography. For example, photolithography cannot be used for synthesis of high aspect-ratio (ratio of height to width) structures. Diffusional limitations arising from photopolymerization also make this approach unsuitable for the synthesis of features with size less than $300 \mu .{ }^{19}$

By extending the concept of spatially controlled, in situ synthesis of polymers from FRRPP systems, we have shown that it is, indeed, possible to produce microgel structures (hydrogel microstructures), as small as $10 \mu$, that should be ultrafast responsive due to their size and also synthesis strategy. In addition to NIPAm, the process was also extended to the synthesis of microgels based on poly (methacrylic acid) (PMAA), which is also an ionic hydrogel (that responds to a change in $\mathrm{pH}$ of its ambience) and has an LCST at $42^{\circ} \mathrm{C}^{20}$ Unlike NIPAm, methacrylic acid is strongly ionic and thus readily ionizes upon irradiation, forming hydrogen bonds with water, which initially causes a gel-effect-like condition. However, the polymer-lean phase and the polymer-rich (irradiated zones) phase coexist even after the growing polymer phase separates out of the solvent, thus decreasing the contrast during pattern transfer. At higher temperatures, the spatially constrained irradiation induces surface strain in the microgels (due to phase separation deep in the phase envelope), while the polymer-lean phase easily breaks apart from the exposed zones. This was also confirmed experimentally by comparing the evolution of patterns at low and high doses, for the same aspect ratio. PNIPAm precipitates relatively quickly out of solution, and the resultant polymer grows in the exposed regions. At low doses, the formed polymer will still be of low molecular weight and will not have completely excluded the solvent. As the overall dose or time of exposure is increased, the patterns spatially align themselves with the exposed regions since the increase in their molecular weight drives out the solvent alienating them to form 
a polymer-rich environment in the exposed zones. This is quite similar to the self-assembly behavior of block copolymers or polymer-polymer blends with an LCST.

The effect of various factors, such as the absorbed energy, thickness of the patterns, and temperature, on the pattern replication aspects of this technique was qualitatively studied by optical microscopy [Chapter 5]. The effect of synthesis conditions on product properties were qualitatively deduced from the change in response rates of PNIPAm microgels synthesized at different doses and temperatures. Since microgels are not large enough to study by small-angle scattering ${ }^{\S}$, bulk gels were prepared by radiation-induced polymerization in a retrograde-precipitation environment. The only difference between the microgels and the bulk gels is the absence of an x-ray mask in the latter, which slightly changes the absorption spectrum of the monomers and thus their morphology, for the same dose. The response of bulk gels will still be similar to those of microgels, albeit at slightly lower doses. The response of PNIPAm bulk gels was characterized by small-angle neutron scattering (SANS) as a function of temperature [Chapter 6]. The process is also described from an application perspective, in which the process methodology more than the reaction chemistry is deemed important [Chapter 7]. PNIPAm microgels also appear not to be spatially conforming to the areas of irradiation. This is due to the postfacto thermoreversible distortions in PNIPAm and should not have any bearing on the reaction control aspects of FRRPP. This hypothesis was also supported by the excellent pattern replication aspects of PMAA, which does not induce any structural deformations due to a change in temperature.

\footnotetext{
$\S$ Sample for SANS should at least be of $0.5 \mathrm{~mm}$ in diameter.
} 


\section{Synthesis of Nanocomposite Microgels}

Since the process methodology is a simple irradiation of the monomers in a poor solvent and at a particular temperature, this approach is quite flexible in its applicability. For example, by polymerizing the monomers in the presence of another polymer dissolved in the solvent, one can produce grafted copolymer microgels. One can also envision the synthesis of nanocomposite microgels, by polymerizing the monomers in the presence of metal nanoparticles incorporated in a poor solvent environment. These aspects are discussed as a special section in Chapter 5 . 


\section{References}

1. Caneba, G. T., Adv. Polym. Tech, 1992, 11, 277.

2. Aggarwal, A., Saxena, R., Wang, B., Caneba, G. T., J. App. Polym. Sci., 1996, 62, 2039.

3. Wang, B., Dar, Y. L., Shi, L., Caneba, G. T., J. App. Polym. Sci., 1999, 71, 61.

4. Dar, Y. L., Caneba, G. T., Chem. Eng. Commun., 2002, 189, 571.

5. Dar, Y. L., Caneba, G. T., Chem. Eng. Commun., in press.

6. Dar, Y. L., PhD Thesis, 1999, Michigan Technological University, Houghton, MI.

7. Tobolsky, A. V., Macromolecules, 1958, 80, 5927.

8. Tobolsky, A. V., Macromolecules, 1960, 82, 1277.

9. Marten, F. L., Hamielec, A. E., ACS Symposium Series, 1979, 104, 43.

10. Chiu, W. Y., Carratt, D. S., Soong, D. S., Macromolecules, 1983, 16, 348.

11. Achilias, D., Kiparissides, C., J. Appl. Polym. Sci., 1988, 35, 1303.

12. Saraf, A. B., Gupta, D. N., Gupta, S. K., Polym. Eng. Sci., 1995, 35, 1290.

13. Chapiro, A., Radiation Chemistry of Polymeric Systems, Interscience Publishers, Great Britain, UK, 1962.

14. Hirasa, O., Ito, S., Yamauchi, A., Fujishige, S., Ichijo, H., Polymer Gels, Ed. DeRossi, D. et al., Plenum, New York, NY, 1991. 
15. Kabra, B. G., Gehrke, S. H., Polym. Commun., 1991, 32, 322.

16. Wu, X. S., Hoffman, A. S., Yager, P., J. Polym. Sci.: Part A, 1992, 30, 2121.

17. Walther, D. H., Blanch, H. W., Prausnitz, J. M., LBL Report No. 34875, UC-401, Lawrence Berkeley Laboratory, Berkeley, CA, 1993.

18. Beebe, D. J., Moore, J. S., Bauer, J. M., Qing, Y., Liu, R. H., Devadoss, C., ByungHo, J., Nature, 2000, 404, 588-590.

19. Liu, V. A., and Bhatia, S. N., Biomedical Devices, 4:4, Kluwer Academic Publishers, Netherlands, 2002, 257.

20. Aggarwal, A., 1993, M. S. Thesis, Michigan Technological University, Houghton, MI. 


\section{Chapter 2}

\section{A Novel Sparse Matrix Representation of Free Radical Polymerization Simulations}

\section{Introduction}

Free radical polymerization (FRP) reactions exhibit a range of complex kinetic events. The conversion of monomer to polymer significantly changes the density and viscosity of the reaction mixture, which affect the transport properties and reaction kinetics. These changes are strongly dependent on the polymer concentration, as well as on polymer chain length and chain length distribution. Several researchers ${ }^{1}$ have tried to model FRP and its associated thermodynamic and kinetic phenomena by trying to account for such changes explicitly. They did so by considering polymer species of different chain lengths to be individual reactive species and modeled the kinetics of polymerization by solving the mass balance equations for all the species. ${ }^{2}$ This leads to thousands, even millions, of equations in complex polymerizations, necessitating simplification procedures for this approach to be computationally viable.

This problem was partially addressed by the method of moments (MOM) approach, which was computationally less intensive. This approach involves the transformation of 
the mass balance equations for all polymeric species to equations modeling moments of the molecular weight distribution (typically the zeroth, first and second moments). ${ }^{3}$ The solution of these equations can accurately simulate well-behaved polymerization reactions with certain limitations. These limitations have restricted the application of this approach to modeling complex polymerization processes. For example, MOM can only accurately describe polymerizations with unimodal molecular weight distributions and cannot account for shoulders or multiple peaks in molecular weight distributions that are usually associated with many FRP systems. In addition, the actual dependence of rate coefficients on system parameters cannot be modeled explicitly using the MOM approach. This can result in the failure of this approach to precisely model even relatively simple real-world systems. ${ }^{4}$

Averaging procedures have been suggested ${ }^{5}$ to overcome this limitation, but this problem cannot be solved unless this dependence can be factored explicitly for individual chain lengths. The use of averaging approaches results in a loss of detail in the simulation of systems with multimodal or broad molecular weight distributions, as seen in many industrial products. Several researchers have used averaging approaches combined with the MOM approach. Teymour et $a t^{6,7}$ developed the so-called numerical fractionation (NF) techniques to overcome the limitations of the MOM approach and applied it to gelforming systems. Butte et al. modified the method developed by Kumar and Ramakrishna (KR method) $)^{8}$ to predict chain length distribution in bulk ${ }^{9}$ and emulsion ${ }^{10}$ polymerization systems. Both the NF and KR methods use the MOM for small partitions of the chain length distributions to overcome the problems associated with the approach. Though these methods have been successful at providing detailed chain length 
distribution information, the most accurate description can only be obtained by solving all the population balance equations for each individual species in the system. ${ }^{7}$

The approach described here, based on a sparse-matrix ${ }^{11}$ representation of the polymerization kinetics, is directed towards modeling FRP in a way that allows for the explicit consideration of the effect of chain length on kinetic parameters, while facilitating the application of suitable computational techniques that can reduce the time and effort needed for the simulation. A sparse matrix is one that has a large number of zero elements ( $>50 \%)$. Use of sparse-matrix-specific solution techniques can greatly simplify the storage and CPU requirements for solving large numbers of equations in such a model. The first step in the use of these techniques is the development of an efficient sparse matrix representation of the mass balance equations. This representation can then be subjected to sparse matrix manipulation techniques like partitioning and tearing to reduce solution effort and time. ${ }^{12,13}$ These techniques use concepts from graph theory ${ }^{14}$ to model information flows in large systems of equations. ${ }^{15,16,17}$ The approach described in this communication is amenable to such solution methods and could serve as a powerful tool in the modeling, simulation, and understanding of chain polymerization processes.

\section{Free Radical Polymerization Kinetics}

Free radical polymerization is a type of chain polymerization, wherein monomer repeat units add on to a propagating radical chain. Steps in this process include chain initiation, typically initiated by a radical initiator activated either by temperature or light (UV); chain propagation (addition of monomer units); chain transfer to monomer, solvent, initiator, polymer, or chain transfer agent; and chain termination by combination or 
disproportionation. The details for each step are well-known and have been widely discussed in the literature. ${ }^{18}$ Recapping briefly, a typical free radical polymerization reaction comprises the following steps:

Initiation

$\mathrm{I} \rightarrow 2 \mathrm{R} \bullet+\mathrm{Gas}$

$\mathrm{R} \bullet+\mathrm{M} \rightarrow \mathrm{P}_{1}$

$2 \mathrm{R} \bullet \rightarrow \mathrm{I}^{\prime}$ (recombined inactive initiator molecule),

where $\mathrm{I}$ is the initiator molecule, $\mathrm{R} \bullet$ is the primary initiator free radical, $\mathrm{M}$ is a monomer molecule, and $\mathrm{P}_{1}$ is the polymer radical of chain length one. Typically the initiator fragment is not included when describing the polymer chain length. The gas is typically nitrogen or oxygen, evolved during an elimination reaction leading to radical generation from azo-compounds or peroxides.

Propagation

$\mathrm{P}_{\mathrm{n}}+\mathrm{M} \rightarrow \mathrm{P}_{\mathrm{n}+1}$,

where $P_{n}$ is a polymer radical of chain length $n$ and $P_{n+1}$ is a radical of chain length $n+1$.

Termination

$\mathrm{P}_{\mathrm{n}}+\mathrm{P}_{\mathrm{m}} \rightarrow \mathrm{D}_{\mathrm{n}}+\mathrm{D}_{\mathrm{m}} \quad$ (Disproportionation)

$\mathrm{P}_{\mathrm{n}}+\mathrm{P}_{\mathrm{m}} \rightarrow \mathrm{D}_{\mathrm{n}+\mathrm{m}}, \quad$ (Combination)

where $D_{m}, D_{n}$, and $D_{n+m}$ are polymer chains (not radicals) of chain lengths $m, n$, and $m+n$, respectively. 


\section{Chain Transfer}

$\mathrm{P}_{\mathrm{n}}+\mathrm{CT} \rightarrow \mathrm{D}_{\mathrm{n}}+\mathrm{CT} \bullet$,

where $\mathrm{CT}$ is the chain transfer agent, which could be a monomer molecule, a polymer chain, an impurity, or a chain transfer agent added to increase transfer rates.

Modeling and simulation approaches for the reaction thermodynamics and kinetics of FRP have also been extensively reviewed. ${ }^{1}$ The approach used to model the polymerization kinetics is described briefly in the next section.

\section{Modeling Free Radical Kinetics}

The kinetics of free radical polymerization are conventionally modeled as a set of mass (or mole) balance equations. These ordinary differential equations (ODEs) can be solved individually for each polymer species or converted into equations based on the moments of the polymer chain length distribution. Models for kinetic rate coefficients, sometimes called "gel-effect" models, are used to determine the rates of propagation and termination reactions, as a function of polymer concentration, chain length, and other reaction parameters. During polymerization, the system density changes with an increase in conversion as the monomer and polymer densities are typically different.

Chain transfer has been neglected in the following analysis, but can be added as an additional term in the mass balance for each polymer species if it is important in the reaction schema, as discussed by Gao and Penlidis. ${ }^{19}$ The following equations represent the kinetics of this system: ${ }^{20}$ 


$$
\frac{1}{V} \frac{d([I] V)}{d t}=-k_{d}[I]
$$

$$
\frac{1}{V} \frac{d([M] V)}{d t}=-k_{p}[M] P
$$

$$
\frac{1}{V} \frac{d([R] V)}{d t}=2 f k_{d}[I]-k_{t}[R][M]
$$

$$
\frac{1}{V} \frac{d\left(\left[P_{l}\right]\right)}{d t}=k_{i}[R][M]-k_{p}[M]-k_{t}\left[P_{l}\right] P
$$

$$
\frac{1}{V} \frac{d\left(\left[P_{n}\right] V\right)}{d t}=k_{p}[M]\left(\left(\left[P_{n-l}\right]\right)-\left[P_{n}\right]\right)-k_{t}\left(\left[P_{n}\right]\right) P
$$

$$
\frac{1}{V} \frac{d\left(\left[D_{n}\right] V\right)}{d t}=k_{t d}\left[P_{n}\right] P+\frac{1}{2} k_{t c} \sum_{m=1}^{n-1}\left[P_{m}\right]\left[P_{n}\right]
$$

$$
\mathrm{x}=\frac{[\mathrm{M}]_{0} \mathrm{~V}_{\mathrm{o}}-[\mathrm{M}] \mathrm{V}}{[\mathrm{M}]_{0} \mathrm{~V}_{0}}
$$

$$
\mathrm{V}=\frac{\mathrm{V}_{0}}{(1+\beta)}(1-\varepsilon \mathrm{x}+\beta)
$$




$$
\varepsilon=\frac{\left(\rho_{p}-\rho_{m}\right)}{\rho_{p}}
$$

$$
\beta=\frac{f_{s}}{\left(1-f_{s}\right)}
$$

$$
f_{s}=\frac{V_{\text {solvent }}}{V_{0}} .
$$

where,

[I] - Initiator concentration

[M] - Monomer concentration

[R] - Primary initiation radical concentration

$\left[\mathrm{P}_{1}\right]$ - Primary polymer radical concentration

$\left[\mathrm{P}_{\mathrm{n}}\right]$ - Concentration of polymer radical of length $\mathrm{n}$

$\left[D_{n}\right]$ - Concentration of dead polymer molecule of length $n$

$\left[\mathrm{M}_{0}\right]$ - Initial monomer concentration

x - Conversion

$\mathrm{V}_{0}$ - Initial volume

$\mathrm{V} \quad$ - Volume

$\mathrm{V}_{\text {solvent- }}$ " Volume of solvent

$\varepsilon \quad-\quad$ Relative change in density as defined above

$\beta \quad-\quad$ Relative fraction of solvent defined as $f_{s} /\left(1-f_{s}\right)$

$\mathrm{f}_{\mathrm{s}} \quad$ - Fraction of solvent volume

$\rho_{\mathrm{p}} \quad$ - Density of polymer 


$\begin{array}{ll}\rho_{\mathrm{m}} \quad-\quad & \text { Density of monomer } \\ \mathrm{k}_{\mathrm{p}} \quad-\quad & \text { Propagation rate constant } \\ \mathrm{k}_{\mathrm{tc}} \quad-\quad & \text { Rate constant for termination by combination } \\ \mathrm{k}_{\mathrm{td}} \quad-\quad & \text { Rate constant for termination by disproportionation } \\ \mathrm{k}_{\mathrm{t}} \quad-\quad & \text { Rate constant for termination (combination and disproportionation) } \\ \mathrm{k}_{\mathrm{d}} \quad-\quad & \text { Rate constant for initiation }\end{array}$

\section{A Novel Sparse Matrix Representation}

Equations (2-1)-(2-11) can be converted to a set of four mass-balance vector ODEs (equations (2-12)-(2-15)) after some manipulation. The assembly of matrices used in these equations can then be implemented on a computer using relatively straightforward matrix manipulations ${ }^{21}$. Derived originally from the material balance equations for all the species in a polymerization process, these equations completely address the kinetics of free radical polymerization. As explained earlier, chain transfer and other side reactions can also be added as separate terms to equations (2-12)-(2-15) when applicable. All one-dimensional matrices are denoted by a single bar (-) over the variable associated with that matrix, whereas all two-dimensional matrices are denoted by double bars $(=)$ over the variable name, as typically used in vector notation. All symbols used have been described earlier, except $\lambda_{0}$, which is the sum of the concentrations of all polymer radicals of all chain lengths.

$$
\begin{gathered}
\frac{d[I]}{d t}=\left\{-k_{d}+\frac{\varepsilon k_{p}(1-x) \lambda_{0}}{(1-\varepsilon x+\beta)}\right\}[I] \\
\frac{d x}{d t}=k_{p}(1-x) \lambda_{0}
\end{gathered}
$$




$$
\begin{gathered}
\frac{d[\bar{P}]}{d t}=\overline{\bar{A}} \cdot\left[\overline{P^{*}}\right]-\left(k_{t d}+k_{t c}\right) \bar{P} \cdot \bar{I}_{n, 1}^{T} \cdot[\bar{P}]+\frac{\varepsilon k_{p}(1-x) \lambda_{0}}{(1-\varepsilon x+\beta)}[\bar{P}] \\
\frac{d[\bar{D}]}{d t}=k_{t d} \bar{Q} \cdot \bar{I}_{1, n} \cdot[\bar{P}]+k_{t c} \cdot \overline{\bar{C}} \cdot[\bar{P}]+\frac{\varepsilon k_{p}(1-x) \lambda_{0}}{(1-\varepsilon x+\beta)}[\bar{D}]
\end{gathered}
$$

Where,

$$
\begin{aligned}
& =\left[\begin{array}{cccccc}
2 f k_{d}[I] & -k_{p}[M] & 0 & \cdot & \cdot & 0 \\
0 & k_{p}[M] & -k_{p}[M] & 0 & \cdot & \cdot \\
& 0 & \cdot & \cdot & \cdot & \cdot \\
& & \cdot & \cdot & \cdot & \cdot \\
& 0 & k_{p}[M] & -k_{p}[M] & 0 \\
0 & & k_{p}[M] & -k_{p}[M]
\end{array}\right] \quad \bar{Q}=\left[\begin{array}{c}
P_{0} \\
P_{1} \\
\cdot \\
\cdot \\
P_{n} \\
0 \\
\cdot \\
0
\end{array}\right] \\
& \overline{I_{1, n}}=\left[\begin{array}{lllll}
1 & 1 & \cdot & 1
\end{array}\right]
\end{aligned}
$$

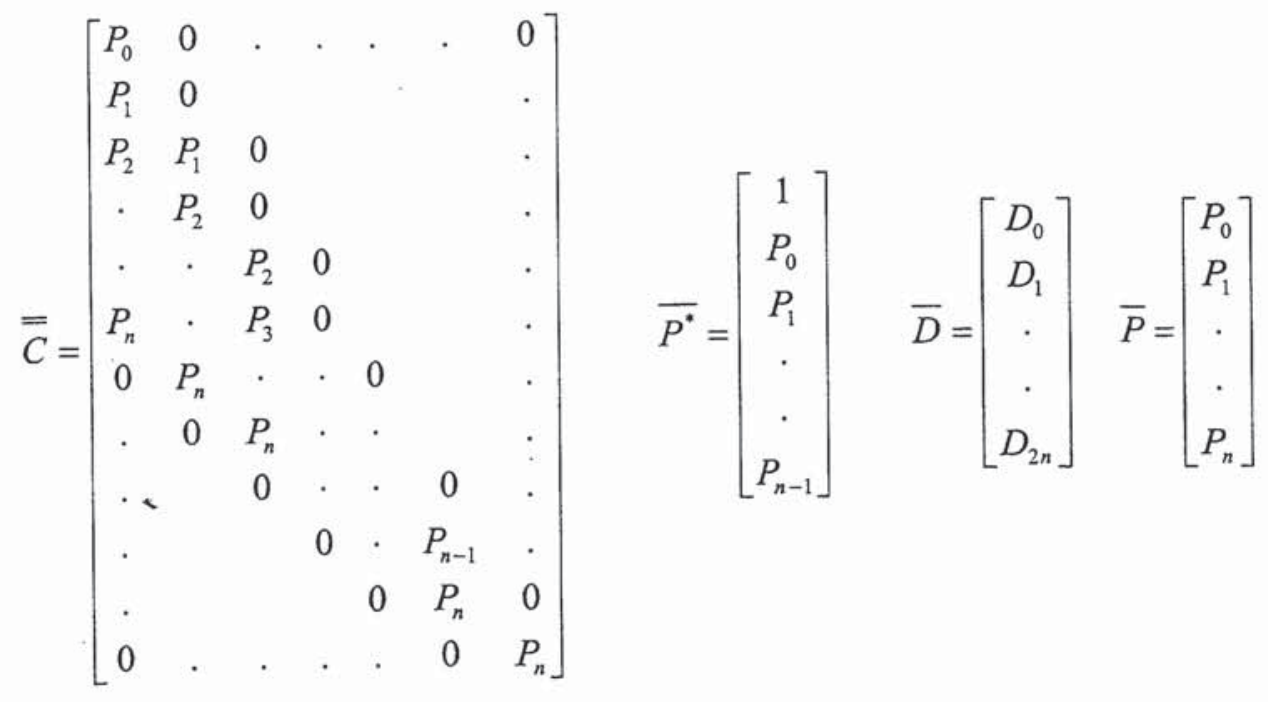


Most of the matrices used here are one dimensional and require minimum computing resources for both storage and processing. Bottlenecks in computation will mainly arise from handling the larger two-dimensional (2-D) matrices. It can be seen that the large 2D matrices used (A and $C$ ) are sparse with occupancies much lower than 50\%. These equations can now be solved on a platform that facilitates vector or parallel solution techniques, along with a suitable numerical algorithm and methods for the use of sparse matrix techniques.

The dimensions of each matrix used are shown in Table 2.1 as a function of $n$, the chain length of the longest active radical chain in this process. Note that the maximum chain length of any molecule is twice $n$, which would be created due to termination by combination.

Table 2.1. Dimensions of Matrices Used in Equations (2-12)-(2-15).

\begin{tabular}{|l|l|}
\hline Matrix & Dimension (row x column) \\
\hline A & $(n+1) \times(n+1)$ \\
\hline C & $(2 n+1) \times(n+1)$ \\
\hline D & $(2 n+1) \times 1$ \\
\hline I & $1 \times n$ \\
\hline P & $(n+1) \times 1$ \\
\hline P* & $(n+1) \times 1$ \\
\hline Q & $(2 n+1) \times 1$ \\
\hline
\end{tabular}

The occupancy of matrices $\mathrm{A}$ and $\mathrm{C}$ can be calculated using equations 2-16 and 2-17 respectively. 


$$
\begin{aligned}
& \text { Occupancy for } A=\frac{2 n}{(n+1)^{2}} \\
& \text { Occupancy for } C=\frac{n+2}{4 n+2}
\end{aligned}
$$

It can be seen that matrix $\mathrm{A}$, being a predominantly block diagonal matrix, has very low occupancy at higher values of $n$ (from 0.039 for $n=50$, to 0.0002 for $n=10,000$ ), whereas matrix $\mathrm{C}$ has an average occupancy of 0.25 for all $\mathrm{n}$.

\section{Results and Discussion}

The approach was validated by simulating the bulk polymerization of methyl methacrylate ${ }^{16}$ based on the parameters used by Chiu et al (Table 2.2). ${ }^{22}$ Simulations were run in a MATLAB (Mathworks, Inc) environment on a Sun Blade 1000 processor.

Table 2.2. Parameters Used for the Simulation of Methyl Methacrylate Bulk

\begin{tabular}{|c|c|c|}
\hline Parameter & Value & Source \\
\hline$\rho_{\text {MMA }}$ & $0.973-1.164 \times 10^{-3}(\mathrm{~T}-273.16)$ & \multirow[t]{7}{*}{ Chiu et al. ${ }^{22}$} \\
\hline$\rho_{P M M A}$ & $1.2 \mathrm{gm} / \mathrm{cc}$ & \\
\hline $\mathrm{MW}_{\mathrm{MMA}}$ & $88 \mathrm{gm} / \mathrm{mol}$ & \\
\hline $\mathrm{k}_{\mathrm{p} 0} \quad-$ & $2.95 \times 10^{7} \mathrm{e}^{(-4353 / \mathrm{RT})} \mathrm{L} / \mathrm{mol} \mathrm{sec}$ & \\
\hline $\mathrm{k}_{\mathrm{t} 0}$ & $5.88 \times 10^{9} \mathrm{e}^{(-70 \mathrm{~T} / \mathrm{RT})} \mathrm{L} / \mathrm{mol} \mathrm{sec}$ & \\
\hline $\mathrm{k}_{\mathrm{d}}$ & $6.32 \times 10^{16} \mathrm{e}^{(-15430 / \mathrm{T})} 1 / \mathrm{sec}$ & \\
\hline F & 0.58 & \\
\hline $\mathrm{T}$ & $363.16 \mathrm{~K}$ & As selected \\
\hline
\end{tabular}
Polymerization 
The simulation results for conversion vs. time and $\mathrm{kp} / \mathrm{kt}$ calculations used for the model are shown in Figure 2.1. The simulation results have been compared to experimental data generated by Marten and Hamielec. ${ }^{23}$ The reaction rate coefficients were modeled using the model developed by Chiu et al (CCS model). ${ }^{22}$ The parameters used in modeling the rate coefficients are reproduced in Table 2.3 for reference. The dependence of kinetic rate coefficients on these parameters is given as: ${ }^{22}$

$$
\begin{aligned}
& k_{p}=\frac{k_{p 0}}{\left[1+k_{p 0} \theta_{p} \lambda_{0} \exp \left(-\frac{2.3 \phi_{m}}{A+B}\right)\right]} \\
& k_{t}=\frac{k_{t 0}}{\left[1+k_{t 0} \theta_{t} \lambda_{0} \exp \left(-\frac{2.3 \phi_{m}}{A+B}\right)\right]}
\end{aligned}
$$

Table 2.3: Parameters for Calculation of Rate Coefficients (From Chiu et $a l^{22}$ ).

\begin{tabular}{|l|l|l|l|l|l|}
\hline Pzn Temp, ${ }^{\circ} \mathrm{C}$ & {$\left[\mathrm{I}_{0}\right]$} & $\Theta_{\mathrm{t}}, \mathrm{min}$ & $\Theta_{\mathrm{p}}, \mathrm{min}$ & $\mathrm{A}$ & $\mathrm{B}$ \\
\hline 50 & 0.0258 & $1.5 \mathrm{e} 3$ & $3.5 \mathrm{e} 3$ & 0.134 & 0.03 \\
\hline 50 & 0.01548 & $2.33 \mathrm{e} 3$ & $3.5 \mathrm{e} 3$ & 0.134 & 0.03 \\
\hline 70 & 0.0258 & 49 & $2.5 \mathrm{e} 2$ & 0.152 & 0.03 \\
\hline 70 & 0.01548 & 83 & $2.5 \mathrm{e} 2$ & 0.152 & 0.03 \\
\hline 90 & 0.0258 & 3.8 & 30 & 0.163 & 0.03 \\
\hline 90 & 0.01548 & 6.3 & 30 & 0.163 & 0.03 \\
\hline
\end{tabular}

The CCS model was chosen to model the rate coefficients as it has been used by the creators to adequately predict the polymerization system simulated here. The model and its successors were also highly successful in simulating a variety of FRP systems despite 


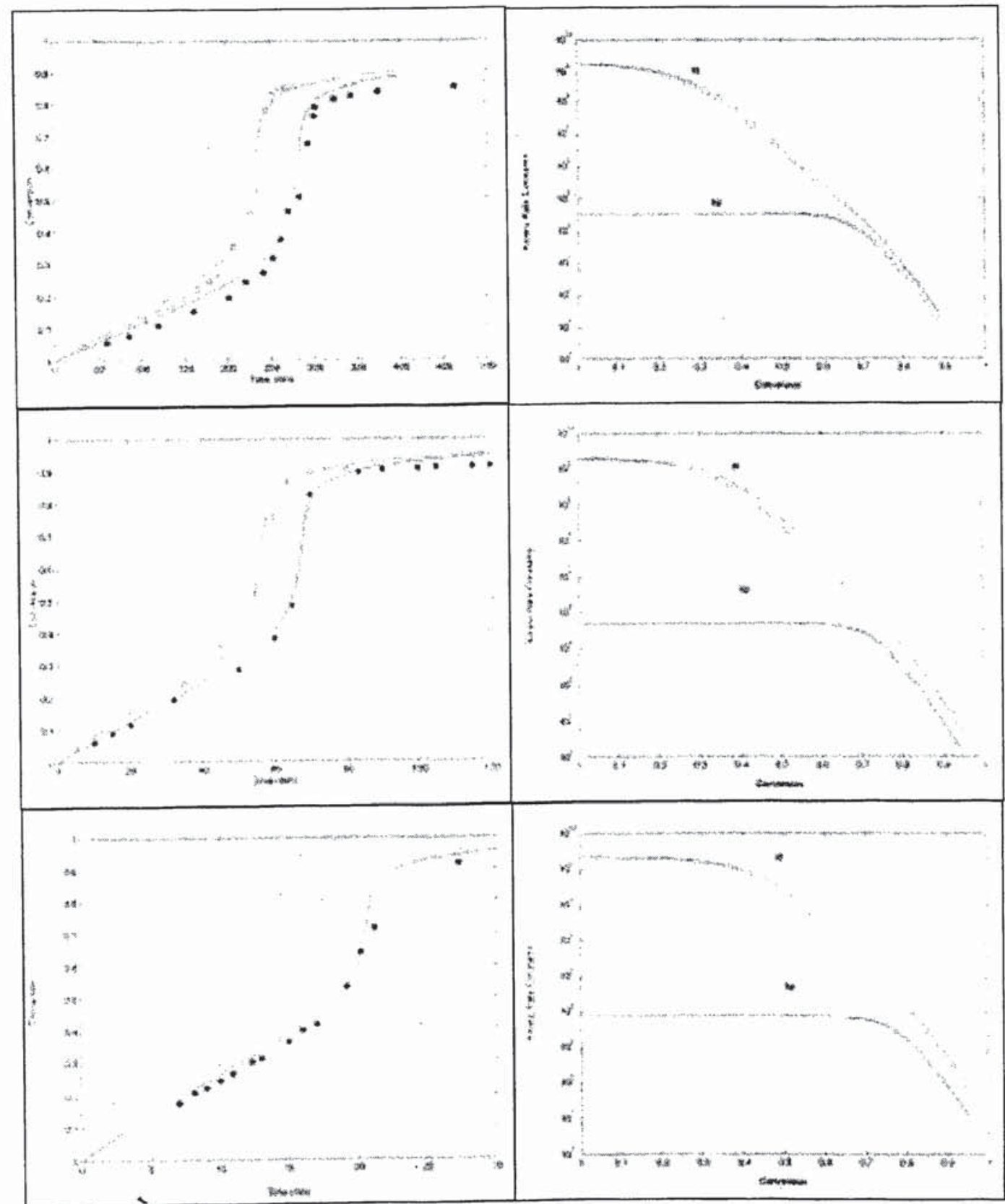

Figure 2.1: Conversion (left) and kinetic rate coefficient (right) histories of MMA bulk polymerization at $50^{\circ} \mathrm{C}$ (top panel), $70^{\circ} \mathrm{C}$ (middle), and $90^{\circ} \mathrm{C}$ (bottom panel), at two initiator loadings: $\left[\mathrm{I}_{0}\right]=0.0258 \mathrm{~mol} / 1$ (hollow markers) and $0.01548 \mathrm{~mol} / 1$ (solid markers). Markers are experimental values ${ }^{23}$ and lines are simulation results. 
a basic flaw in wrongly assigning the boundary conditions for a polymerization chain reaction event. ${ }^{24}$ Other models have been shown to have superior simulation capabilities that are applicable to more generic systems. ${ }^{24,25}$ It was also relatively simple to integrate into the model without any complexities and is a good choice to verify the validity of the current approach. Incorporating more complex models gives us more information concerning the actual kinetic events, as needed for the system being simulated. Note that the conversion-time data correlates well with the experimental data, even during the onset of the gel effect. The rate coefficients change as the volume fraction of polymer increases in the reaction. The results agree well with the simulation results presented by Chiu et al.

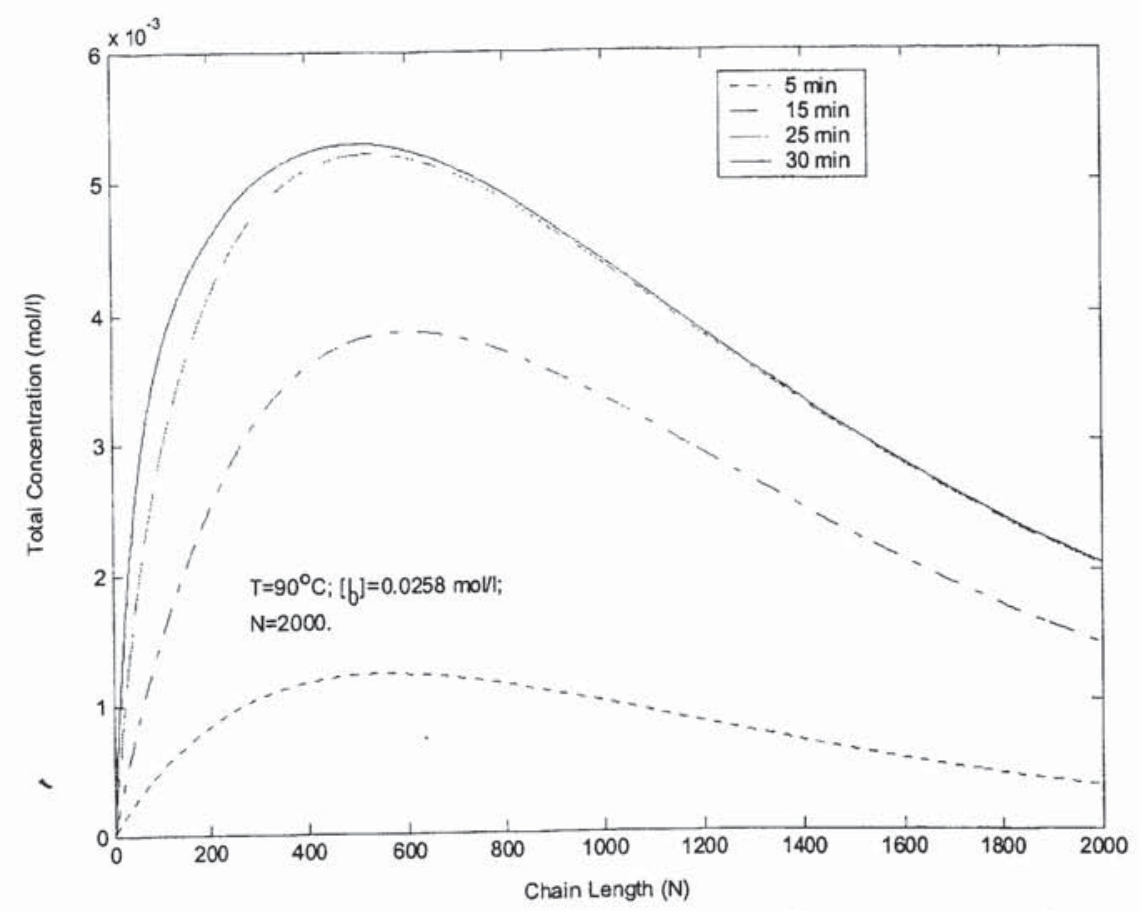

Figure 2.2: Population distributions at various times of MMA polymerization at $90^{\circ} \mathrm{C}$ and at an initiator loading of $0.0258 \mathrm{~mol} / \mathrm{l}$.

The chain length distributions for all polymeric species in the system (newly generated 
radicals as well as terminated polymer) for reaction times of $5,15,25$, and 30 minutes are shown in Figure 2.2. The corresponding chain length distributions for the terminated polymer molecules and the propagating free radicals are shown in Figures 2.3 and 2.4, respectively. The data represent the evolving polymer chain length distribution as a function of time and can be used to obtain precise information about the chain length distribution at any given time period. Note that the average chain lengths are much lower compared to an actual system since the distributions are all clipped at $\mathrm{N}_{\max }$.

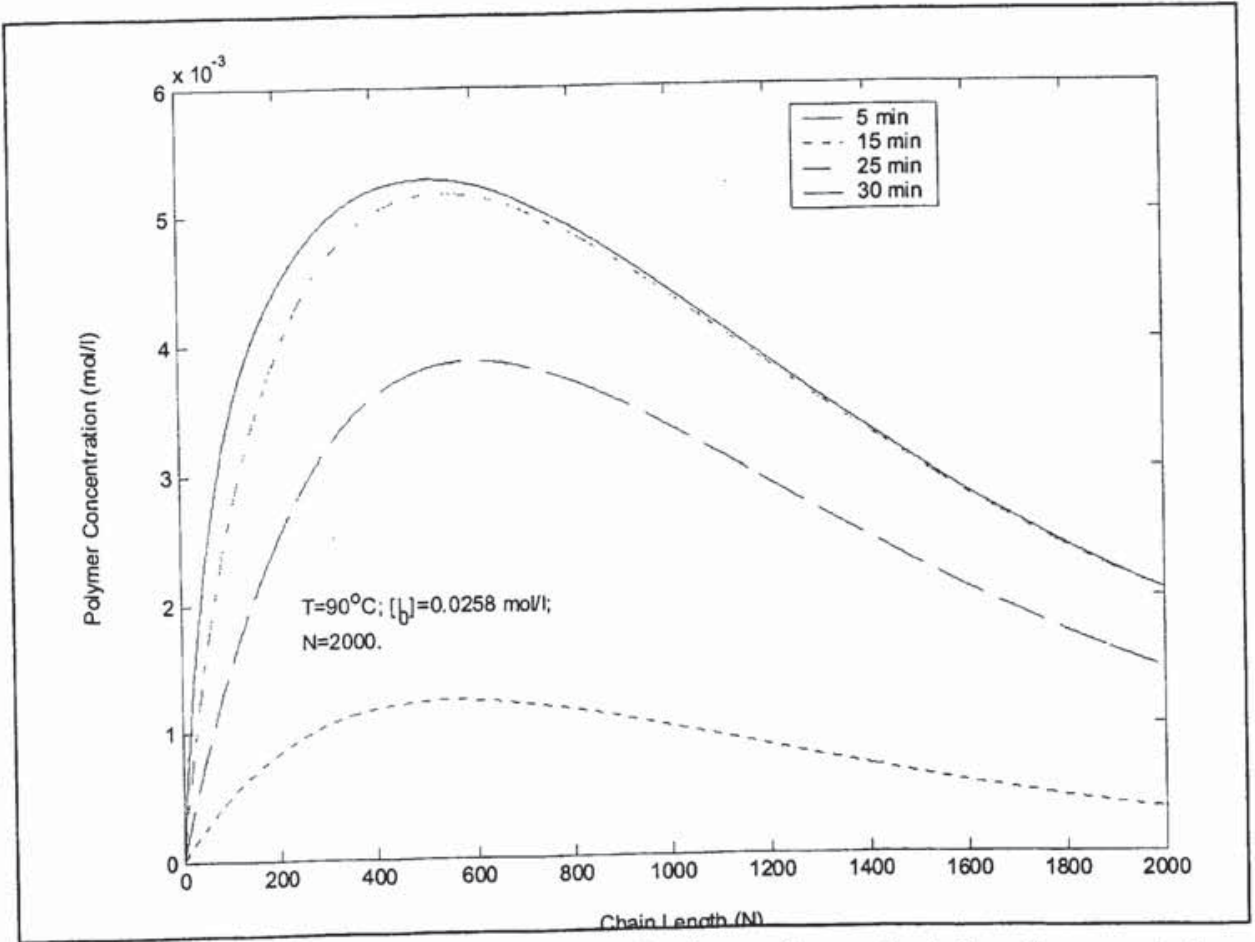

Figure 2.3: Simulated chain length distribution of terminated polymer chains at various times of reaction during MMA bulk polymerization at $90^{\circ} \mathrm{C}$, and $\left[\mathrm{I}_{0}\right]=$ $0.0258 \mathrm{~mol} / \mathrm{l}$.

Even though these data are significantly influenced by the restrictions on $\mathrm{N}_{\max }$ they can still be used to make some interesting observations about the kinetics of this system. The 
data shown in Figures $2.2-2.4$ are from initial stages in the polymerization. It can be seen that the polymer population at lower chain lengths increases due to a high rate of initiation during the gel-effect phase. It is also interesting to note that, once the onset of the gel effect begins, the most significant increase is in the populations of smaller chain length propagating radicals, as seen in Figure 2.4.

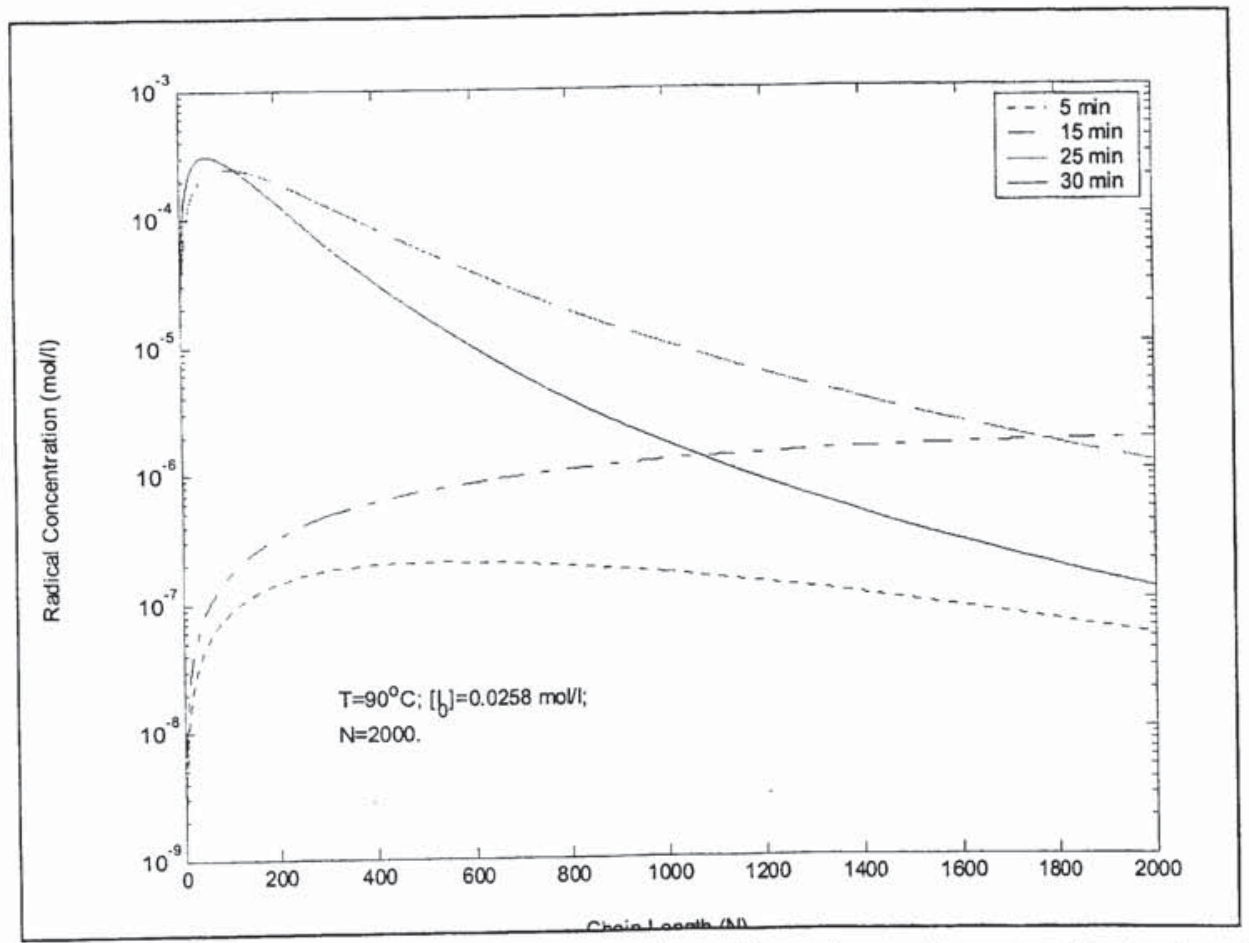

Figure 2.4: Simulated chain length distribution of growing polymer radicals at various times during the bulk polymerization of $\mathrm{MMA}$ at $90^{\circ} \mathrm{C}$ and $\left[\mathrm{I}_{0}\right]=0.0258 \mathrm{~mol} / \mathrm{l}$.

This is believed to be a feature of the high rate of initiation in this system, since, at $90^{\circ} \mathrm{C}$, the half-life of the initiator used is about 10 minutes, and the gel-effect starts even before two half-lives of the initiator. The radical chain length distribution plot in Figure 2.4 shows a corresponding rise in the population of polymer radicals of smaller chain lengths. Thus, the decrease in the rates of termination observed in Figure 2.1 strongly affects the lower chain length region significantly due to the high rates of chain 
generation. The longer chain lengths are also affected in the real system, but that impact is not observed here due to a limitation on $\mathrm{N}_{\max }$.

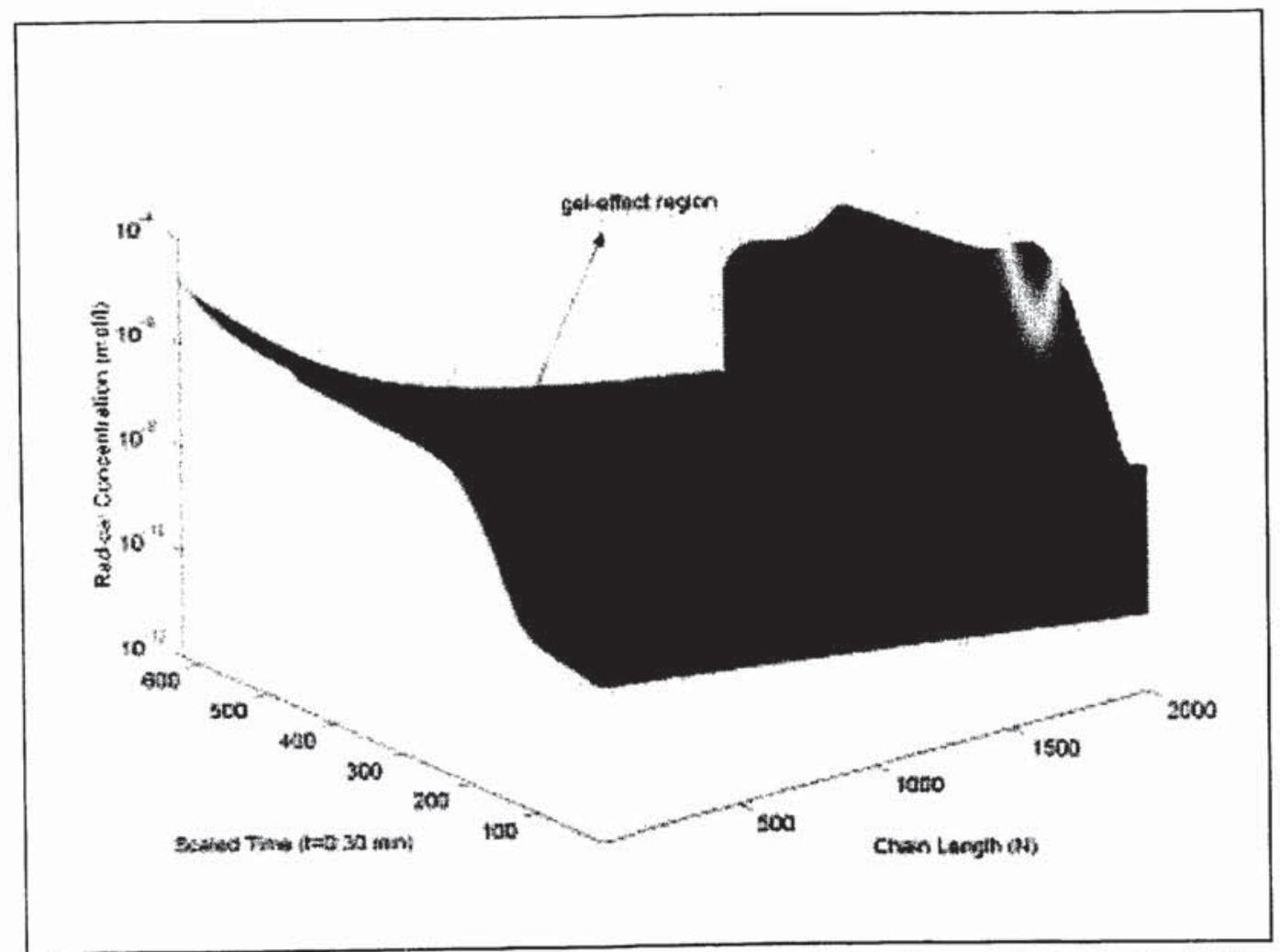

Figure 2.5: Simulated concentration profile of the growing polymer chains in the bulk polymerization of MMA at $90^{\circ} \mathrm{C}$ and $\left[\mathrm{I}_{0}\right]=0.0258 \mathrm{~mol} / \mathrm{l}$.

The concentration evolution profiles of polymer radicals can be directly obtained from the simulation results (Figure 2.5). The actual inherent values for the chain lengths are much longer than the values used here and therefore results in artifacts as explained earlier. The large population of radicals seen at the maximum chain length is due to the accumulation of unreacted polymer radicals at the limiting $\mathrm{N}_{\max }$ value. These radicals would actually have contributed to the high molecular weights observed in the gel-effect 
region of a real system, if they were allowed to propagate beyond the maximum allowed chain length.

It should be noted that this level of detail is only available from this approach and cannot be obtained from MOM-based calculations or other similar averaging procedures. Such complete information also leads to a better understanding of polymerization kinetics, especially in more complex systems. Modeling of the evolution of the chain lengths was found to be strongly influenced by $\mathrm{N}_{\max }$, the maximum allowable chain length in the process. The choice of $\mathrm{N}_{\max }$ is, therefore, an important parameter in the simulations. The chain lengths of the polymer molecules produced during a polymerization reaction are determined by kinetic parameters like temperature, type and concentration of the monomer and initiator. However, the high memory requirements of the current method limit the maximum matrix size used in simulations, corresponding to an $\mathrm{N}_{\max }$. This is a significant limitation since the maximum available chain length in simulations should be sufficiently large enough to be able to capture the entire spectrum of chain length distribution in the system. The value of $\mathrm{N}_{\max }$ should also be larger than the longest chain length that exists in the physical system for the parameters chosen. In fact, $\mathrm{N}_{\max }$ should, ideally, be twice as large as the longest polymer radical to account for termination by bimolecular combination. Nevertheless, this approach is best studied to simulate special cases of free radical polymerizations, in which inherent chain lengths are relatively smaller compared to conventional polymerizing systems. ${ }^{26,27,28}$

The CPU times required for simulation also increase with a cubic dependence on $\mathrm{N}_{\max }$, as seen in Figure 2.6. The in-house system used in this study could not satisfy the memory required for simulations of optimally required $N_{\max }(\sim 10,000)$. The choice of lower values results in an artifact, narrowing the molecular weight distribution with an 
artificially imposed upper limit at the $\mathrm{N}_{\max }$ value chosen. Future work in this area will include using superior computational resources as well as using partitioning and tearing methods that reduce the requirements on memory and CPU time.

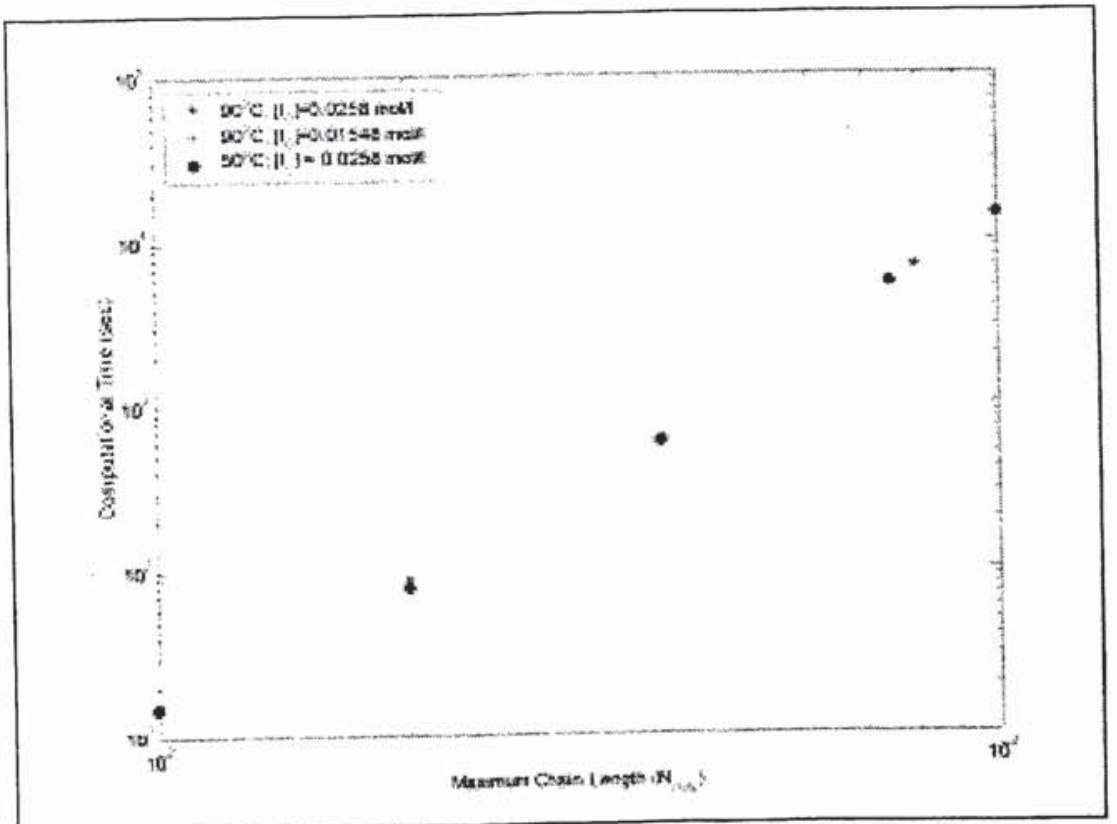

Figure 2.6: Variation of computation times with the maximum available chain lengths, at different temperatures and initiator loading given in the legend.

\section{Conclusions}

A computationally efficient, sparse-matrix-based representation of FRP was developed. The methodology was validated by comparing the simulation results from this approach with those of other models discussed in the literature and also with experimental values. The results correlate very well with both the experimental and simulation data, for the chosen test system. Apart from matching the usual conversion profiles widely used in the 
literature to measure the success of a model, our approach also gives detailed kinetic information on the population distribution of the system at any given time.

The high levels of computation time and memory needed for the simulation has led to imposing artificial limits on the chain lengths in the system, which means that the molecular weight data cannot be compared to experimental data or other simulation data at this time. The information currently available from the results is significant enough warranting the application of this approach in FRP simulations all the same. This includes information on the population distribution as a function of initiator decomposition and monomer conversion. The incorporation of different rate coefficient models, including the ones explicitly dependent on chain lengths, ${ }^{29}$ should also be relatively straightforward with this approach. In addition, including information on initiator decomposition over time with a more explicit dependence on chain length and reaction environment should be significantly straightforward. This is possible only due to the decoupled representation of the kinetic equations, where chain length dependent rate coefficient values can be generated in a matrix form and used in the system as developed.

Future work will be targeted towards taking advantage of some of these features. We are currently developing the appropriate level of computer resources as well to incorporate sparse matrix manipulation techniques for simulation using large values $(>10,000)$ of $N_{\max }$. 


\section{References}

1. Dotson, N. A., Galvan, R., Laurence, R. L, Tirrell, M., Polymerization Process Modeling, VCH Publishers, New York, 1996.

2. Skeirik, R. D., Grulke, E. A., Chem. Eng. Sci., 1985, 40, 535.

3. Venkateshwaran, G., Kumar, A., J. App. Poly. Sci., 1992, 45, 187.

4. Dar, Y. L., Caneba, G. T., Chem. Eng. Comm., 2002, 189, 571.

5. Casey, B. S., Mills, M. F., Sangster, D. F., Gilbert, D. F., Napper, D. H., Macromolecules, 1992, 25, 7063.

6. Teymour, Campbell, J. D., Macromolecules, 1994, 27, 2460.

7. Papavasiliou, Birol, I., Teymour, F., Macromol. Theory Simul., 2002, 11, 533.

8. Kumar, S., Ramakrishna, D., Chem. Eng. Sci., 1996, 51, 1311.

9. Butte, Storti, G., Morbidelli, M., Macromol. Theory Simul., 2002, 11, 22.

10. Butte, Storti, G., Morbidelli, M., Macromol. Theory Simul., 2002, 11, 37.

11. Himmelblau, D. M., Chem Eng. Sci, 1966, 21, 425.

12. Tarjan, R. E., SIAM J. Comput., 1972, 1, 146.

13. Steward, D. V., J. SLAM Numer. Anal. B, 1965, 2(2), 345.

14. Harary, F., Graph Theory, Addison-Wesley, New York, 1969. 
15. Kernighan, W., Lin, S., Bell Syst. Tech. J., 1970, 49, 291.

16. Liu, J. W. H., ACM Trans. Math. Soft., 1989, 15(3), 196.

17. Pothen, Fan, C. J., ACM Trans. Math. Soft., 1990, 16(4), 303.

18. Odian, G., Principles of Polymerization, John Wiley and Sons, New York, 1991.

19. Gao, J., Penlidis, A., J.M.S.-Rev. Macromol. Chem. Phy., 1996, C36, 199.

20. Aggarwal, A., Saxena, R., Wang, B., Caneba, G. T., J. App. Polym. Sci., 1996, 62, 2039.

21. Dar, Y. L., Ph.D. thesis, Dept. Chem. Eng., Michigan Technological University, Houghton MI, 1999.

22. Chiu, Y., Carratt, G. M., Soong, D. S., Macromolecules, 1983, 16, 348.

23. Marten, F. L., Hamielec, A. E., ACS Symposium Series, 1979, 104, 43.

24. Litvinenko, G. I., and Kaminsky, V. A., Prog. Reaction Kinetics, 1994, 19, 139.

25. Saraf, B., Gupta, D. N., Gupta, S. K., Polym. Eng. Sci., 1995, 35, 1290.

26. Tobolsky, A. V., Macromolecules, 1958, 80, 5927.

27. Tobolsky, A. V., Macromolecules, 1960, 82, 1277.

28. Caneba, G. T., Adv. Polym. Tech, 1992, 11, 277.

29. Russel, G. T., Gilbert, R. G., Napper, D. H., Macromolecules, 1992, 25, 2459. 


\section{Chapter 3}

\section{Nanoparticles from Quiescent FRRPP Systems}

\section{Introduction}

Nanoparticles are subject to active research recently owing to their high surface areato-volume ratio or high void surface area compared to that of a bulk material. Polymeric nanostructures have many advantages over their metal counterparts in the applications such as cosmetics, optical barriers, colorants, coatings, pesticides, lubricants, electrophotography, biosensors, and microelectromechanical systems (MEMS). Nanostructured materials are typically prepared from inorganic/organic precursors, either by gas phase synthesis or sol-gel processing. These techniques usually result in nanopartickes with diameters ranging from 1-10 nm, with consistent crystalline structure and high degree of monodispersity. Additional synthesis techniques for nanoparticle preparation include sonochemical processing, cavitation processing, microemulsion processing $^{1,2}$ and precipitation polymerization. ${ }^{3}$ 
Only recently, the synthesis of nanostructured materials from block copolymers ${ }^{4-6}$ was reported. Researchers have succeeded in the preparation of block copolymer nanostructures from a variety of polymers like polystyrene (PS), poly (2-cinamoylethylmethacrylate) (PCEMA), poly (acrylic acid) (PAA), poly (t-butyl acrylate) (PtBA) and polyisoprene (PI). ${ }^{6}$ Based on these polymers, block copolymeric nanostructures were prepared, such as hollow nanospheres, star polymers, tadpole

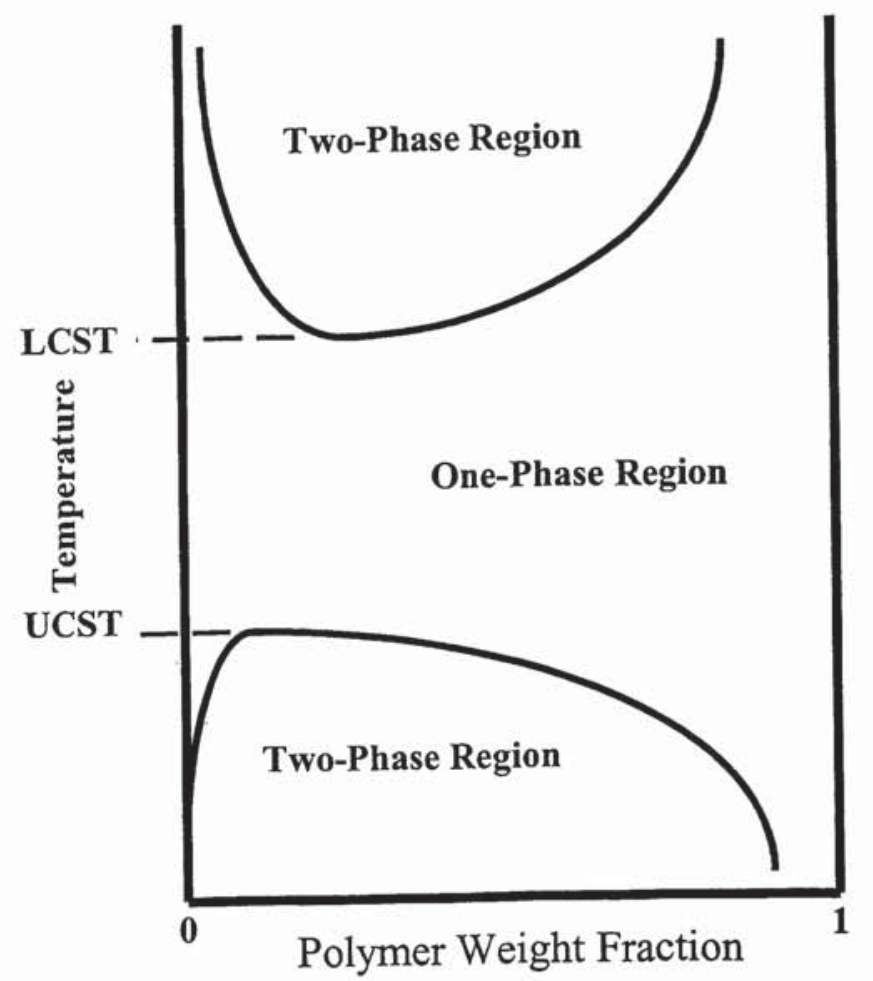

Figure 3.1: Binary amorphous polymer-solvent phase diagram showing the possibilities of phase separation below the upper critical solution temperature (UCST) and above the lower critical solution temperature (LCST).

molecules, polymer brushes, nanochanneled fibers, etc., as reported in literature..$^{7-9}$ Most of these nanostructures were prepared from diblock micelle crosslinking using thermal ${ }^{10}$ or photoinitiators. ${ }^{11}$ Of the numerous applications possible, polymeric nanostructures are 
being investigated for stealth drug carriers in drug delivery systems ${ }^{12-14}$ and synthesis of metal nanoclusters. ${ }^{15-17}$ However, most of these techniques process premade polymers to obtain nanoparticles: a "top-down" approach. Apart from a few fabrication techniques, like chemical vapor deposition, electrodeposition, electron-beam or x-ray lithography, there are not many methods that use a "bottom-up" approach.

In this chapter, we describe a new way of fabricating nanoparticles in situ from a reaction, in the absence of any additives like surfactants or post-processing methods. The method is based on the so-called free radical retrograde-precipitation polymerization (FRRPP) process, ${ }^{18-20}$ wherein free radical chain polymerization occurs along with phase separation above the lower critical solution temperature (LCST) (see Figure 3.1).

From our early work, ${ }^{18}$ we proposed the following mechanism of the FRRPP process:

1. Gradual increase of conversion vs. time even under gel-effect conditions

2. Local heating around the radical site

3. Reduced rate of propagation as well as the rate of radical-radical termination

4. Relatively narrow molecular weight distributions

5. The existence of live radicals that could be exploited for production of block copolymers.

Recently, we have been able to elucidate some of the underlying concepts behind this mechanism. ${ }^{20}$ We found that the exotherm of the polymerization reaction raises the local temperatuxe of the reaction zone to the spinodal curve of the phase diagram, whereby mutual diffusivities have been found to vanish. This results in the slowdown of the propagation reaction, until the reaction zone temperature drops to a condition a little below the spinodal curve. Of course, further propagation will raise the reaction zone temperature toward the spinodal curve again. When this incremental heating and cooling 
in the vicinity of the spinodal curve occurs continually, the result is a control of the propagation of chains. The precipitated condition also results in the slowdown of polymer radical termination, if conditions are such that chain transfer is minimized. In a more recent work, ${ }^{21}$ we showed that the approach of the reaction zone to the spinodal curve is not the only mechanism of reaction control. It seems that higher local temperature is not a necessary condition for reaction control. We have shown in this work that even with small enough domain sizes where heat dissipation is expected to be relatively fast, the rate of polymerization is under good control leading to the stabilization of nanosized globular precipitates.

If the reaction rate is under good control, the polymer-rich domain growth will also be under good control if domain interaction is minimized. Thus, in a mixed vessel reactor, some nanometer-sized polymer particles should be obtainable if a crosslinker is included with the reaction recipe. If the reaction is carried out in a quiescent fluid, then we should expect a large amount of these particles.

Atomic force microscopy (AFM) is a widely used characterizing tool for polymeric microstructures. Many researchers ${ }^{22-25}$ have successfully used AFM to directly observe microstructures such as metal, polymeric, and composite nanoparticles, polymer gels, etc. Together with a complimentary tool such as transmission electron microscopy (TEM), small angle scattering, complete information on a given system can be obtained. synchrotron small angle $\mathrm{x}$-ray scattering (SAXS) is an excellent tool to elucidate material properties of structures ranging from polymeric solutions, gels, and composites to biological macromolecules. The use of SAXS to investigate large-scale polymer structures is nicely summed up in a recent review. ${ }^{26}$ In our work, we have used AFM for the direct observation of polymeric nanoparticles while using SAXS for the in situ 
observation of nanoparticle formation. The reactive systems used in the techniques are, however, different because of the difficulty associated with in situ observation of highpressure reactive systems.

\section{Experimental}

\section{Materials Used}

Styrene (S), ethylene glycol dimethacrylate (EGDMA) and methacrylic acid (MAA) monomers were purchased from Aldrich Chemical Co. Styrene and methacrylic acid were double distilled under reduced pressure to remove inhibitor, while EGDMA was used as-is. Analytical grade diethyl ether solvent and n-heptane were purchased from Fisher Scientific, Inc. 2,2'-azobisisobutyronitrile (AIBN) initiator was purchased from Eastman Kodak, Inc., and V-50 initiator was purchased from Wako Chemicals. Both were used as-is. Milli $Q^{\circledR}$ water was used in all aqueous systems. All fluids used in the reactor were bubbled with nitrogen for at least 15 minutes in order to get rid of the dissolved oxygen. Quartz capillary tubes with a $1.5 \mathrm{~mm}$ I.D. used in SAXS studies were purchased from Charles - Supper, Inc.

\section{Polymerization of Styrene/EGDMA in a Stirred-Tank Reactor}

The apparatus for the polymerization of styrene in diethyl ether was described elsewhere. ${ }^{20}$ In order to impart some dimensional integrity to the polymer, EGDMA crosslinker was added into the polymerization mixture at a level of $10 \mathrm{wt} \%$ relative to the styrene-EGDMA charge. The detailed experimental procedure is described below: 
The reactor was purged with nitrogen initially and was filled with about $150 \mathrm{ml}$ of ether, which was maintained at $80^{\circ} \mathrm{C}$ and $60 \mathrm{psig}$. The reactive mixture containing $0.28 \mathrm{~g}$ of AIBN dissolved in $27 \mathrm{~g}$ of total monomers was pumped into the reactor along with some $18-20 \mathrm{ml}$ ether, at the rate of $5 \mathrm{ml} / \mathrm{min}$. The reactor assembly included a stirrer that was maintained at varying speeds, in order to study the effect of mixing on confining the reaction zones. Within 5 minutes of the reaction initiation, the volume of the ether in the reactor was made up to $200 \mathrm{ml}$. Nitrogen was used to purge the liquid of any leftover material in the lines, along with a 30-32 $\mathrm{ml}$ ether flush. Product samples were collected inside a sealed bottle immersed in an ice bath. After 300 minutes, the reactor fluid was cooled down by a quick flush of cooling water through the cooling coils. Then, the reactor was disassembled, and the final product was collected into a sealed bottle immersed in an ice bath.

In case of homopolymerization of styrene by FRRPP, the reaction was carried out in exactly the same manner as described above, in the absence of EGDMA. Experiments were carried out twice in a similar manner but at varying mixing speeds: one at $50 \%$ of maximum speed ( $300 \mathrm{RPM})$, and the other at $80 \%$ of the maximum speed ( $\sim 500 \mathrm{RPM})$.

\section{Polymerization of Styrene in a Quiescent-Fluid Reactor}

Styrene was polymerized in a $1 / 16^{\prime \prime}-O D$ tube reactor (assumed to closely resemble a quiescent system) using the same recipe as that used in the stirred-tank reactor. A $13.6 \mathrm{~g}$ total weight of monomer mixture was dissolved in solvent along with $0.14 \mathrm{~g}$ of AIBN. Nitrogen was bubbled through the reactive mixture for about 15 minutes, to remove dissolved oxygen. The tube reactor was tightly sealed on one end, while the other end was connected to an Eldex metering pump. Pumping of the reactive mixture was carried 
out for exactly 5 minutes at a rate of $1 \mathrm{ml} / \mathrm{min}$. The other end of the reactor was then sealed, and the tube reactor was immersed in a hot water bath at $80^{\circ} \mathrm{C}$. The reactor product was then pumped out using the same solvent as that of the reaction system. In contrast to an LCST type of reaction, conventional precipitation polymerization was carried out in the tube reactor wherein styrene was polymerized in the presence of nheptane.

\section{Sample preparation for AFM Analysis}

The reactor product was freeze-dried on a silicon wafer, which was initially washed with acetone and toluene to clean up the surface. In order to determine the presence of nanosized particles and their dimensions, an AFM (tapping mode; Nanoscope IIIa from Digital Instruments, Inc.) equipped with a $20-\mathrm{nm}$ etched silicon probe tip was used. Particle analysis of the AFM images was performed using the Nanoscope software.

\section{In situ SAXS Study of Methacrylic Acid Polymerization}

The kinetics of methacrylic acid polymerization in water above the LCST was studied previously in our group. ${ }^{27}$ Since polymerization of styrene in ether is a high-pressure reaction and styrene in t-butyl acetate (TBA)/water is relatively unexplored, we chose the methacrylic acid system for our in situ studies of polymerization. The reaction recipe was as discussed elsewhere. ${ }^{28}$ Quartz x-ray tubes were loaded with reactive mixture containing MAA, water and V-50. The tubes were then vacuum-freeze-thawed in 3.5 cycles, to remove dissolved gases, after which they were flame-sealed under vacuum.

Small angle x-ray scattering analysis of these samples was then carried out at the BESSRC beamline, 11-ID, of the Advanced Photon Source. An in-house sample holder containing a heating element was connected to a temperature controller to help maintain 
the sample at the required temperature. Polymerization was monitored by placing the sample in the holder while simultaneously gathering the SAXS data for a set reaction time, 30 minutes. The reaction was carried out at two different temperatures, $60^{\circ} \mathrm{C}$ and $80^{\circ} \mathrm{C}$. To validate the persistence of size from kinetic experiments, SAXS data from prereacted samples was also obtained. The same reaction recipe ${ }^{28}$ was used to react the samples and for subsequent quenching before they underwent freeze-thaw cycles and flame sealing. These prereacted samples were then placed in the sample holder maintained at reaction temperature, to observe the particle behavior in a simulated reaction environment.

\section{Relevant Concept}

From the polymer physics point of view, the polymerization process can be divided into two main time regimes (Figure 3.2): the initial dilute regime (Stage I) and the twophase regime (Stage II). In turn, Stage I can be subdivided into the isolated chain regime (Stage Ia) and the globular agglomerated chain regime (Stage Ib).

In the dilute stage, the polymerization fluid contains a relatively small amount of isolated polymer coils in the solution of monomer and solvent. The polymerizing chains become increasingly less soluble in the medium as they become long enough to behave more like polymer than monomer. With increasing chain length, the size of the polymer coil becomes increasingly smaller than the size of an equivalent unperturbed polymer chain. This is a result of the so-called coil-globule transition. ${ }^{29-33}$ Note that this transition occurs in systems that are below the theta-temperature in conventional phase separation. 
In inverse-temperature or retrograde phase separation, the coil-to-globule transition occurs above the LCST.

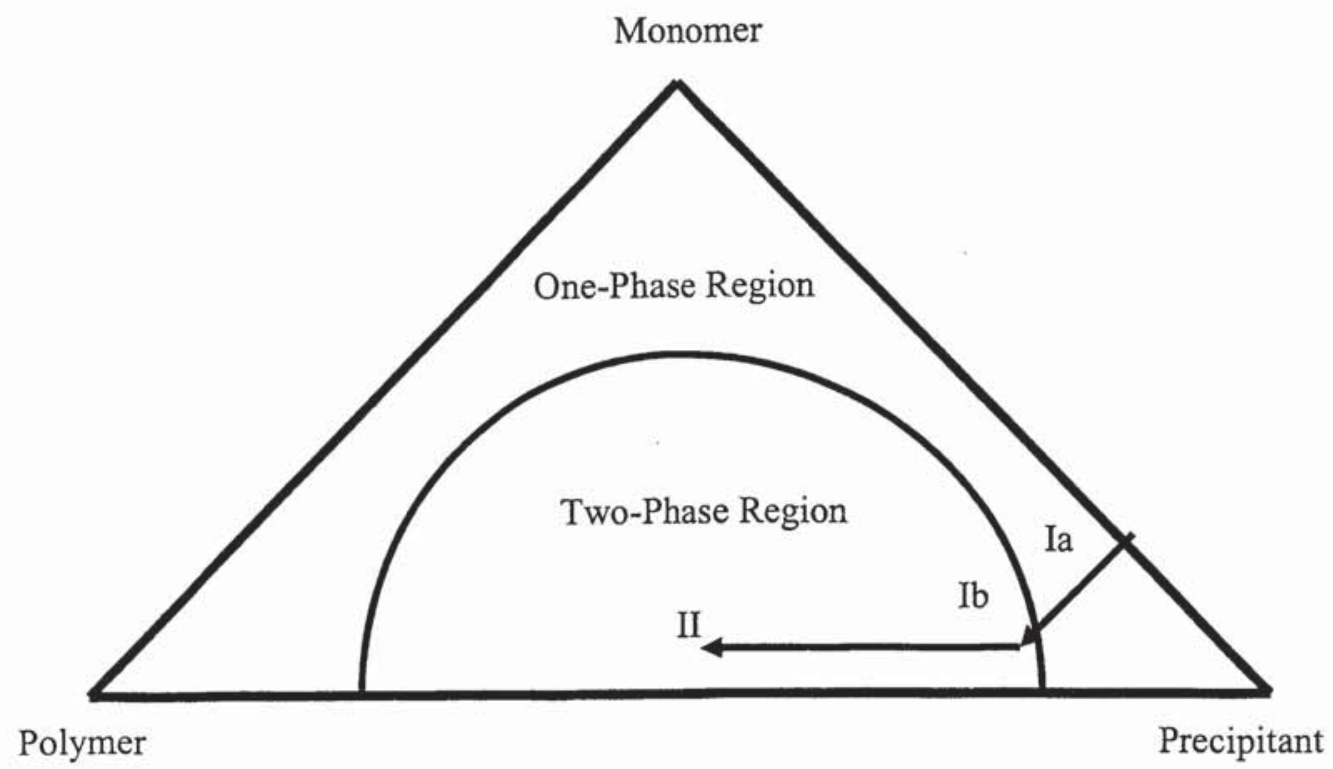

Figure 3.2: Reaction trajectory in a ternary polymer-monomer-solvent phase diagram showing depicted polymer chain configuration at various points in the system: Ia dilute solution regime; Ib - dilute polymer collapsed coil; and II - agglomerated collapsed coil.

This collapse leads to a significant increase in the internal globular volume fraction of the polymer. This is stage Ia. As the concentration of the polymer in the system begins to increase, the individual collapsed globules will collide with each other and agglomerate. This is stage $\mathrm{Ib}$. The behavior in stage $\mathrm{Ia}$ and $\mathrm{Ib}$ is substantially different as mass transfer in stage Ia would be translational diffusion controlled. However, the mass transfer for agglomerated globules or high molecular weight single molecular globules (Stage $\mathrm{Ib}$ ) would better be described by the free volume theory. This is because the presence of more than one polymer chain in the globule would allow further collapse 
due to the screening effect. ${ }^{34}$ Diffusion coefficients within highly collapsed globules will be free-volume controlled. This causes the conversion to rise rather steeply with time initially due to diffusion control of the termination reaction, as it does in a system undergoing the gel effect. As the polymer weight fraction within a globule becomes close to unity, the diffusion can become slower leading to control of the termination as well as the propagation reactions.

As the polymerization progresses leading to an increased polymer weight fraction in the system, collapsed polymer globules will begin to agglomerate to form clusters. When the concentration of the system exceeds a critical value, the system shows widespread agglomeration due to phase separation by spinodal decomposition. ${ }^{20}$ This critical concentration should be closely related to the threshold concentration where the polymer chains begin to interact with each other. This could be analogous to the critical overlap concentration, had the solvent been a good solvent. ${ }^{35}$ With some control over the polymerization kinetics in certain regions within the fluid, it is possible to confine this widespread agglomeration to the immediate vicinity of the reaction zones. This is the aim of our polymerization experiment in a quiescent fluid.

\section{Results and Discussion}

\section{Stirred-Tank Reactor Experiments}

Mixing Speed@300 RPM:

An AFM image from the sample vacuum-dried on a silicon wafer is shown in Figure 3.3. The polymeric nanoparticles in the images can be clearly identified by the difference in color of the particles. The size of the nanoparticles can be observed from the 
intensity of the color of the particles and the height corresponding to that color. It is worth pointing out that this sparse number of nanoparticles is typical of our results from stirred-tank experiments.

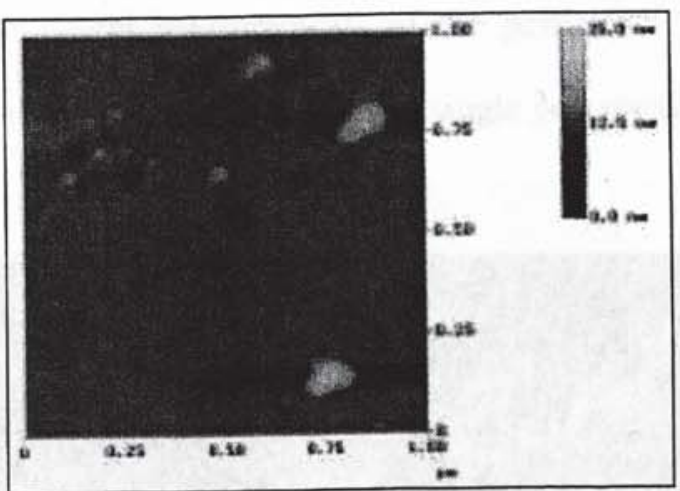

Figure 3.I: AFM topography scan of a freeze-dried product from the stirred-tank reactor experiment. The color scale on the right is applied to the height at each point on the scanned surface.

\section{Mixing Speed @500 RPM:}

At this high speed of mixing, the exotherm of the reaction, distributed throughout the reactor, tends to drive the reaction at a higher rate. This resulted in a solid polymer product. There were no smaller particles ${ }^{1}$ as all the reaction domains were thoroughly interacting leading to coagulated polymer-rich material.

\section{Tube Reactor Experiments}

In Figure 3.4, we show a frame of an AFM scan of nanoparticles that were formed from a quiescent-fluid reaction system. Compared to that of a mixed reactor system (Figure 3.3), the quiescent reactor system (Figure 3.4) has yielded a relatively large number of nanoparticles. The size analysis for different AFM scans of the polymer 
product (shown in Table 3.1), indicates that average sample diameters are in the range of 13-35 nm. Since a relatively blunt (2onm) AFM probe tip was used, the measured diameter is believed to be higher than the actual diameter of the particles. This is evidenced by the smaller height measurements of the particles. Similar results and conclusions were also obtained by Furukawa ${ }^{36}$ from single polystyrene molecules bound on a silicon substrate.

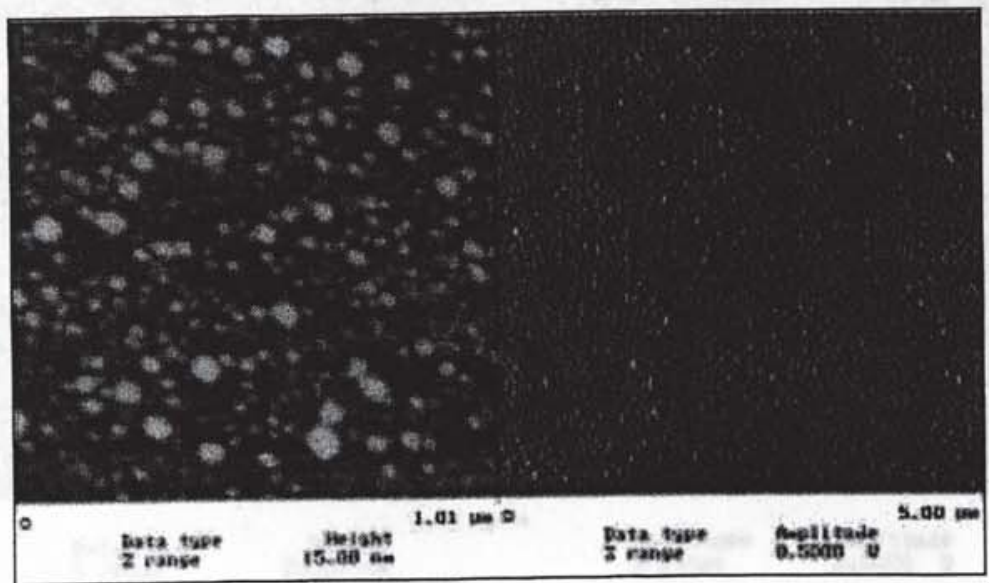

Figure 3.4: AFM scan of resulting nanoparticles from styrene-EGDMA polymerization in ether system, for a reaction time $=40$ minutes. The left frame is based on the height scale given in Figure 3, but with a maximum height of $15 \mathrm{~nm}$. The right frame is the amplitude result from the AFM.

Table 3.1. Average values of particle heights and diameters from the frames of AFM scans for controlled polymerization of styrene-EGDMA in a quiescent tube reactor.

\begin{tabular}{|c|c|c|c|c|}
\hline & Frame 1 & Frame 2 & Frame 3 & Frame 4 \\
\hline Average Height (nm) & 1.4 & 1.2 & 1.7 & 1.2 \\
\hline Average Diameter (nm) & 13 & 21 & 30 & 26 \\
\hline
\end{tabular}


The conventional precipitation polymerization (CPP) system on the contrary, has not resulted in any polymer particles in the nanoscale region. Styrene in the presence of n-Heptane did not precipitate into microscopic polymer domains, clearly differentiating the proposed mechanism of reaction control in FRRPP with that of CPP.

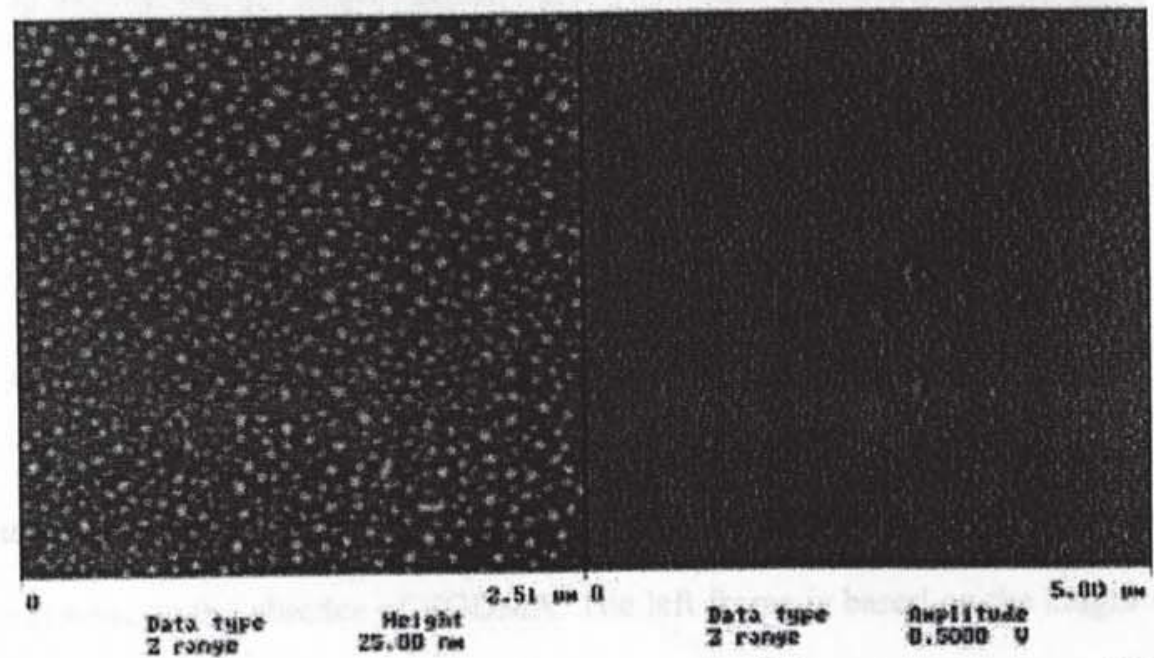

Figure 3.5: AFM scan of resulting nanoparticles from styrene-EGDMA polymerization in TBA-water. The left frame is based on the height scale given in Figure 3. The right frame is the amplitude result from the AFM.

We have recently been able to develop a solvent system that allows us to polymerize styrene at atmospheric pressure. ${ }^{37}$ This is based on the use of a t-butyl acetate/water mixture as solvent, which we found to have an LCST at $55-65^{\circ} \mathrm{C}$. We therefore used the same tube reactor system and procedure to try producing nanosized particles. Figure 3.5 shows that indeed such a solvent system works as well as the ether-based systems in Figures 3.3 and 3.4. We were also able to obtain nanosized homopolymer (no cross-linker in the recipe) particles with almost the same particle size distribution as compared to their crosslinked counterparts (Figure 3.6). The only 
difference is that the uncrosslinked particles are a little deformed, probably due to fluid shearing during transfer from the small tube reactor to the silicon wafer surface.

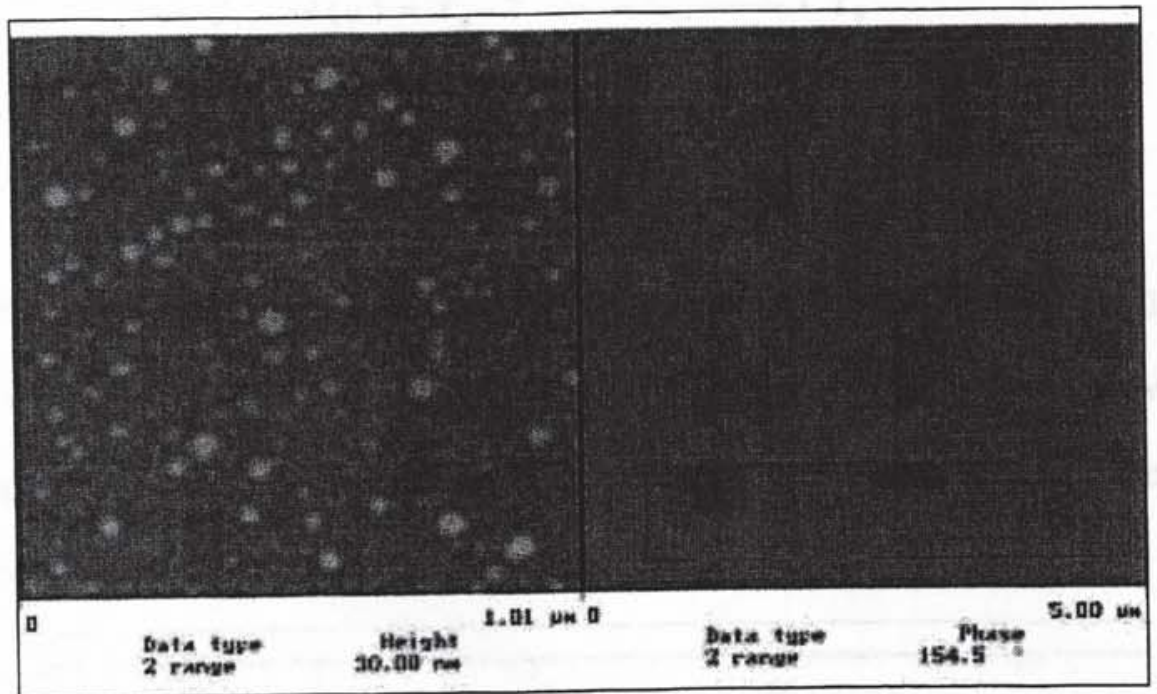

Figure 3.6: AFM scan of resulting nanoparticles from styrene polymerization in ether system, in the absence of EGDMA. The left frame is based on the height scale given in Figure 3, but with a maximum height of $30 \mathrm{~nm}$. The right frame is the amplitude result from the AFM.

Time-Resolved Small Angle X-ray Scattering (TR-SAXS)

The scattering profile from in situ synchrotron SAXS of methacrylic acid polymerization at $60^{\circ} \mathrm{C}$ is shown in Figure 3.7. The data were corrected for a flat background and are plotted as intensity vs. wave vector (Q). From the observed AFM results, it is likely that the polymer particles are spherical in shape. Therefore, at first, idealized data fits such as monodisperse spheres, Shulz-Flory polydisperse spheres, hollow sphere and gaussian chains, were tried to fit intensity as a function of Q. Should these models not fit the data within a reasonable accuracy, it would have been imperative to look at developing new models to fit the data. But as it appears from the data fits, the 
polymeric system in study is best represented by Debye function for a polymer with chain radii similar to that of a gaussian distribution. The functional form is given as:

$$
\begin{gathered}
I(q)=I_{0} \cdot 2 \cdot \frac{\left(e^{-u}+u-1\right)}{u^{2}}+I_{b} \\
\text { and } \\
u=q^{2} \cdot\left\langle R_{g}{ }^{2}\right\rangle ; q=\frac{4 \pi}{\lambda} \operatorname{Sin}\left(\frac{\theta}{2}\right) ;\left\langle R_{g}{ }^{2}\right\rangle=\frac{1}{6} N \cdot l^{2}
\end{gathered}
$$

where, $I$ is the intensity $\left(I_{0} \& I_{\mathrm{b}}\right.$ are blank and background intensities respectively); $q$ is the wave number of the scattered $\mathrm{x}$-rays; $\lambda$ is the wavelength of the incident $\mathrm{x}$-rays; $\theta$ is the angle of scattering; $\left\langle\mathrm{R}_{\mathrm{g}}\right\rangle$ is the mean square radius of gyration; $N$ is the number of monomers; and $l$ is the length of the monomeric unit.

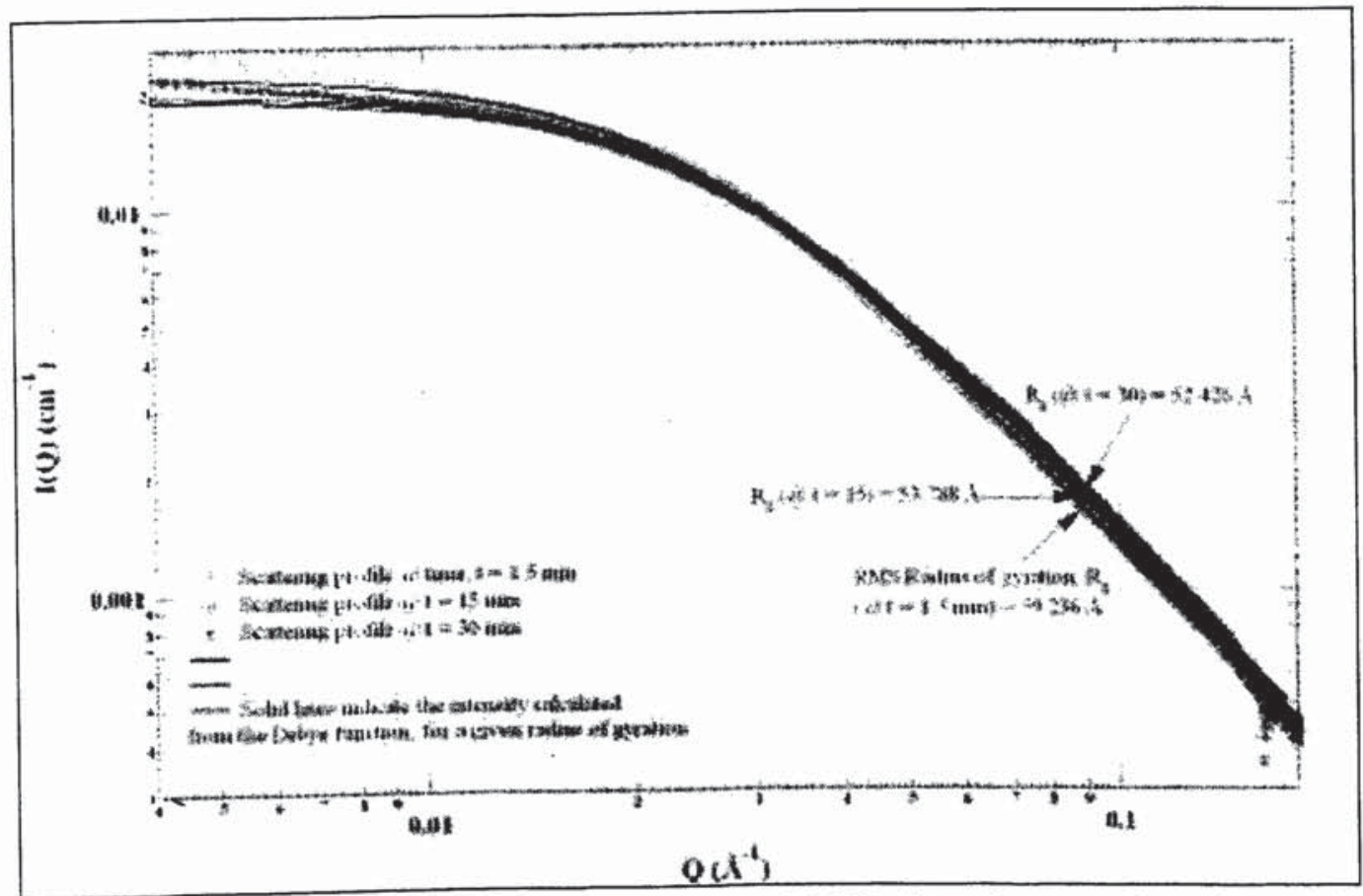

Figure 3.7: Intensity vs. Q plot of in situ SAXS data from methacrylic acid polymerization at $60^{\circ} \mathrm{C}$, obtained as a function of time. The functional form used for the data fit is the Debye function for a random-coiled gaussian polymer. 
This type of gaussian distribution might be appropriate for some anionic polymers with narrow molecular weight distribution data $^{38}$ or for polymer chains in a theta solvent. The in situ SAXS analysis from all the three systems (Figures (3.7)-(3.9)) resulted in data that fit the gaussian distribution function through the entire range of 'low Q', with good accuracy. The radius of gyration, $\mathrm{R}_{\mathrm{g}}$, thus obtained from the data fits is given in Table 3.2. Since the polymer is in a retrograde precipitating environment, we would expect the polymer, in the absence of reaction, to shrink in size as we observe its behavior over a period of time.

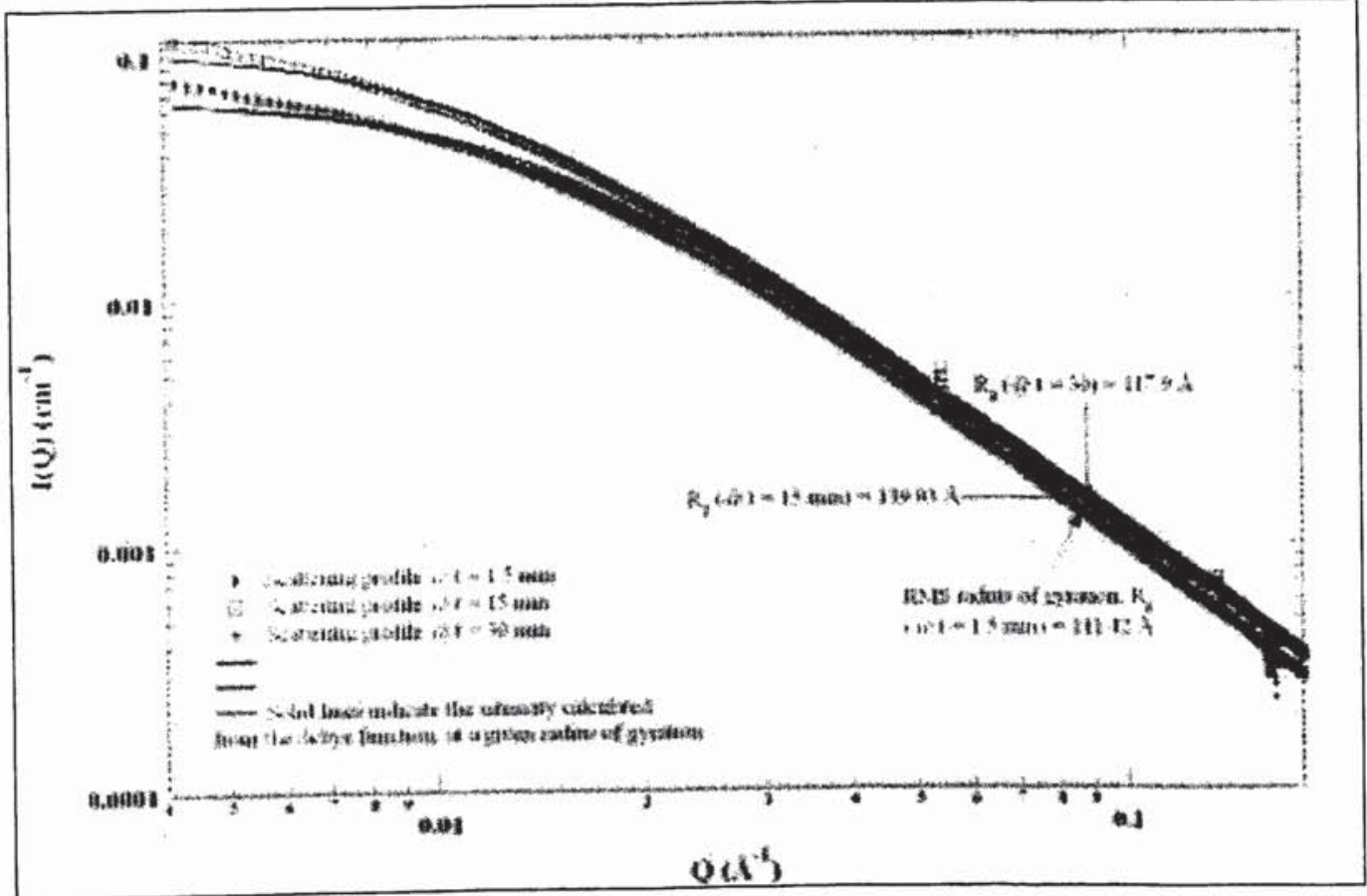

Figure 3.8: Time-resolved, In situ SAXS data of methacrylic acid polymerization at $80^{\circ} \mathrm{C}$, monitored for 30 minutes.

This is particularly true in the case of the pre-reacted system (Figure 3.9), whose average radius of gyration shrank from $126 \AA$ to $97 \AA$. The reaction system is not as simple to predict. The reaction kinetics influence factors such as molecular weight and local 
temperature of the reaction domain, which will have contradictory effects on the size of the polymer. As the reaction proceeds in a domain, the molecular weight

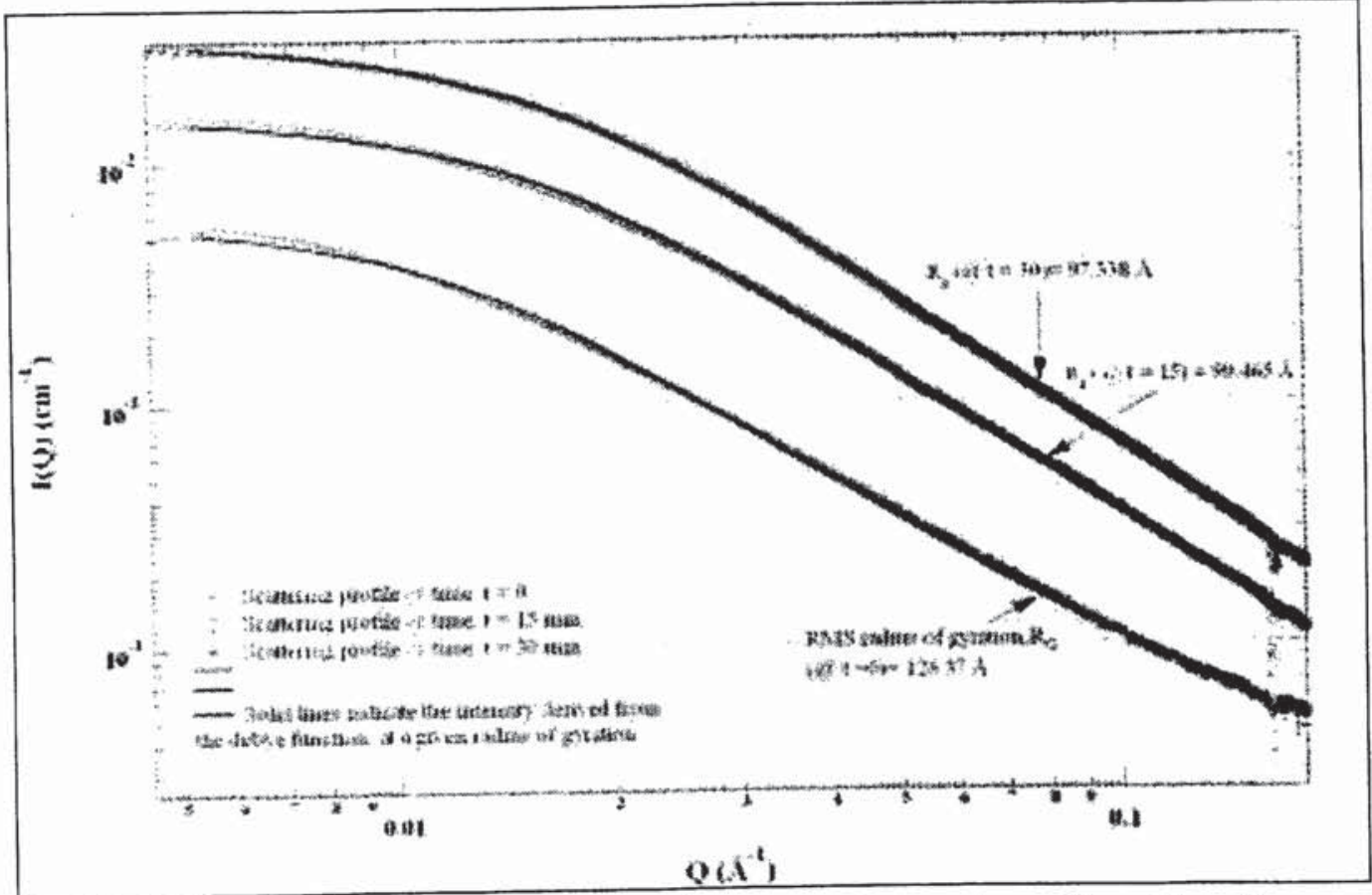

Figure 3.9: SAXS data obtained from the pre-reacted methacrylic acid system at

$60^{\circ} \mathrm{C}$, plotted as a function of time for 30 minutes. Slight changes in the size of the polymer reported.

increases, and, with that, one would normally expect an increase in size of the polymer. But since the reaction is exothermic, the increase in molecular weight also results in an increase in temperature, causing more shrinkage. This, again, is a highly simplified model of the reaction environment. 'More complications arise as we go higher and higher in temperature, which can be explained in light of polydispersity data. The radius of gyration data from the reaction at $60^{\circ} \mathrm{C}$ (Figure 3.7) suggests that the particles have proceeded along the lines as explained above. Nevertheless, the data from reaction at 
$80^{\circ} \mathrm{C}$ (Figure 3.8 ) do not exactly follow the same pattern. Though not surprising, it would be interesting to look into the factors influencing the particle growth at that temperature.

Table 3.2. Radius of gyration obtained from the SAXS data fits for the different FRRPP systems.

\begin{tabular}{|l|l|l|l|}
\hline $\mathrm{R}_{\mathrm{g}}$ after time $\rightarrow,\left({ }^{\circ} \mathrm{A}\right)$ & $\begin{array}{l}1.5 \\
\text { minutes }\end{array}$ & $\begin{array}{l}15 \\
\text { minutes }\end{array}$ & $\begin{array}{l}30 \\
\text { minutes }\end{array}$ \\
\hline Methacrylicacid/Water system, reacting at $60^{\circ} \mathrm{C}$ & 59 & 54 & 52 \\
\hline Methacrylicacid/Water system reacting at $80^{\circ} \mathrm{C}$ & 111 & 139 & 118 \\
\hline $\begin{array}{l}\text { Methacrylicacid/water system pre-reacted for } 15 \mathrm{~min} \\
\text { at } 60^{\circ} \mathrm{C}\end{array}$ & 126 & 100 & 97 \\
\hline
\end{tabular}

\section{Conclusions}

We have shown that nanoparticles can be produced from a controlled precipitation polymerization method without the use of dispersing aids, such as surfactants. Polymerizing the monomer under precipitating conditions above the lower critical solution temperature resulted in reaction control that is shown to have translated to control over the growth of the polymer domains. In a well-mixed fluid, particle-particle agglomeration and polymer chain collapse resulted in only a few surviving small particles. In the absence of mixing, collisions between small individual particles were minimized. This and the controlled rate of polymerization resulted in the preservation of nanosized polymer particles. Unagglomerated polymer nanoparticles were obtained from a quiescent system thus substantiating this concept. The size distribution can be further improved by more instantaneous initiation of polymerization reaction. There is also the 
possibility that these particles can be dried to porous solid materials. Alternately, addition of another hydrophilic monomer in the fluid would cause the formation of a hydrophilic block within the pores and outer surfaces of these nanoparticles. 
Chapter 3: Mixing effects in FRRPP Systems

\section{References}

1. Weihua M., Frank N. J., Shoukuan F., Macromol. Chem. Phys, 1998, 199, 1075.

2. Kapeliouchko V., Maarchese E., 1999, personal communication.

3. Chen, H., Ishizu, K., Fukutomi, T., Kakurai, T., J. Polym. Sci., Polym Chem, $1984,22,2123$.

4. Wilson, D. J., and Reiss, G., Euro. Polym. J., 1988, 24, 2487.

5. Liu, G., Chapter 9, Handbook of Nanostructured Materials and Nanotechnology, 5, Ed. H. S. Nalwa, Academic Press, San Diego, 475, 2000.

6. Liu, G., Smith, C. K., Hu, N., Tao, J., Macromolecules, 1996, 29, 220.

7. Guo, A., Liu, G., Tao, J., Macromolecules, 1996, 29, 2487.

8. Tao, J., Stewart, S., Liu, G., Yang, M., Macromolecules, 1997, 30, 2738.

9. Henselwood, F., Liu, G., Macromolecules, 1997, 30, 488.

10. Saito, R., Ishizu, K., Fukutomi, T., Polymer, 1992, 33, 1712.

11. Tuzar, Z., Bedner, B., Konak, C., Kubin, M., Svobodova, S., Prochazka, K., Makromol. Chemie., 1982, 182, 399. -

12. Bruce Thurmond II, K., Karen L. Wooley, ACS Symposium Series, 1998, 709, Ed. Ian M., Shalaby, W. S., 165. 
13. Gref, R., Minamitake, Y., Peracchia, M. T., Langer, R., Microparticulate Systems for the delivery of Proteins and Vaccines, 77, Ed. Smadar Cohen, Howard Bernstein, Marcel Dekker, Inc., NY, 279, 1996.

14. Alonso, M. J., Nanoparticulate Drug Carrier Technology, Drugs and Pharmaceutical Sciences, 77, Ed. Smadar Cohen, Howard Bernstein, Marcel Dekker, Inc., NY, 203, 1996.

15. Yue, J., Cohen, R. E., Supramolecular Science, 1994, 1, 117.

16. Kane, R. S., Cohen, R. E., Silbey, R., Chem. Mater., 1996, 8, 1919.

17. Sidorov, S. N., Bronstein, L. M., Davankov, V. A., Tsyurupa, M. P., Solodovnikov, S. P., Valetsky, P. M., Wilder, E. A., Spontak, R. J., Chem. Mater., 1999, 11, 3210 .

18. Caneba, G. T., Adv. Polym. Tech., 1992, 11, pp. 277.

19. Aggarwal, A., R. Saxena, B. Wang, and G. T. Caneba, J. App. Polym. Sci., 1996, 62, 2039.

20. Wang, B., Y. Dar, L. Shi, and G. T. Caneba, J. App. Polym. Sci., 1999, 71, 61.

21. Dar, Y. L., Caneba, G.T., Chemical Engineering Communications, 2000, 189, 571.

22. Ros, R., Schewesinger, F., Padeste, C., Pluckthun, A., Anselmetti, D., Guntherodt, H. J., and Tiefenaur, L., Proc. SPIE, 1999, 3607, 84. 
23. Simmons, B. A., Taylor, C. E., Landis, A. A., John, V. T., McPherson, G. L., Shwartz, D. K., and Moore, R., J. Am. Chem. Soc., 2001, 123, 2414.

24. VanLandingham, M. R., Dagastine, R. R., Eduljee, R. F., Mc Cullough, R. L., and Jillespie Jr., J. W., Composites A, 1999, 30, 75.

25. Krause, S., McEvoy, R. L., and Wu, P., Polymer, 1998, 39, 5223.

26. Chu, B. and Hsiao, B. S., Chemical Reviews, 2001, 101, 1727.

27. Aggarwal, A., M.S. Thesis, 1993, Michigan Technological University, Houghton, MI.

28. Aggarwal, A., Saxena, R., Wang, B. and Caneba, G. T., J. App. Polym. Sci., 1996, $62,2039$.

29. Nishio, I., G. Swislow, S. Sun, and T. Tanaka, Nature, 1982, 300, 243.

30. Nierlich, M., Cotton, J. P., and Farnoux, B., J. Chem. Phy., 1978, 69, 1379.

31. Sanchez, I. C., Macromolecules, 1979, 12, 980.

32. Kholodenko, A. L. and K. F. Freed, J. Phy. A: Math. Gen., 1984, 17, 2703.

33. Raos, G. and G. Allegra, J. Chem. Phy., 1996, 104, 1626.

34. De`Gennes, P., Scaling Concepts in Polymer Physics, Cornell University Press, New York, 1979.

35. Strobl, G., The Physics of Polymers, Springer-Verlag, Berlin, 1996. 
36. Furukawa, K., Ebata, K., Matsumoto, N., Fujiki, M., Morita, M., and Torimitsu, K., 2000, Poster presentation during the Eighth Foresight Conference on Molecular Nanotechnology, Bethesda, MD, November 2-5.

37. Kosonen, M.-L., M.S. Thesis, 2001, Michigan Technological University, Houghton, MI.

38. Rennie, A. R., Technical Report, King's College, London, 1996. 


\section{Chapter 4}

\section{Fabrication of Poly (N-isopropylacrylamide)}

\section{Microstructures via a Novel Synthesis Strategy}

\section{Background: Radiation-Induced Polymerization}

Polymerization processes usually require an initiator (chemical species) to initiate the chain reaction. The function of an initiator is either to excite the reactants to form radicals, or to add (remove) an electron from the molecular orbitals to from anionic (cationic) species. Since this result can also be achieved by an irradiative process, radiation was considered an excellent alternative to initiate the organic species, with an added advantage of the product being relatively pure. Radiation induced polymerization processes were well researched for a span of two decades, from the early 50 s to the late 70 s, but the interest died down gradually with only a limited number of participants in the

recent times. Nevertheless, the radiation aspects of polymerization were thoroughly studied as and when new mechanisms of polymerization were discovered. While gammarays are well established as the radiation sources for initiation, electron beams are known only for modifying the product properties and are not used in polymer synthesis. Since the basic principles remain the same irrespective of the initiation source, this study 
focuses on reviewing the gamma-ray induced polymerization only because it is a well-researched component of all the available sources of radiation.

The evolution of radiation chemistry of polymers was chronologically compiled by Chapiro, ${ }^{1}$ which to date remains the only exhaustive review of gamma-ray induced polymerization. Gamma rays and electron beam radiation are widely used to initiate the polymerization reactions since their energy dissipation primarily occurs by molecular excitation and secondary electron generation. The energy absorbed per unit of incident radiation is independent of wavelength within the range of $0.2-2 \mathrm{MeV}$. The incident energy is hence directly proportional to the number of electrons per gram of irradiated material. Absorption of radiation with energy less than $0.2 \mathrm{MeV}$ depends on the chemical composition of the substance, while the absorption of very high energy radiation ( $>2$ $\mathrm{MeV}$ ) requires the knowledge of absorption coefficients and concepts of pair production. Unlike thermal initiation, the type of polymerization in radiation initiation systems cannot be determined beforehand. Polymerization can either be a free radical or an ionic process, depending on the medium of excitation, energy of the radiation, and dose-rates.

\section{Radiation Yield:}

The yield of a reaction is also based on the mechanism of reaction: Ionic yield, defined as a ratio of the number of molecules changed to the number of ions believed to be produced in the system. The yield of free-radical reactions is based on the 'G-values' introduced by Burton, ${ }^{2}$ and is an absolute chemical yield expressed in terms of the number of chemical events occurring per $100 \mathrm{eV}$ of absorbed radiation. Most of radiation

\footnotetext{
* Dose rate is the energy absorbed by the interacting medium per unit time (units: Watts/cc)
} 
reactions are characterized by their yield $G_{R}$, defined as the number of free radicals produced in the systems per $100 \mathrm{eV}$ of absorbed energy. Since radiation initiation deals with excited electrons, the mechanism of propagation and termination in polymeric systems cannot be predicted a priori and have to be experimentally determined.

Gamma-ray Polymerization of Styrene/Acrylates:

Phillips and Hopwood ${ }^{3}$ were the first to use gamma rays to bulk polymerize different monomers in a liquid state: styrene, methyl methacrylate, vinyl acetate etc. Many researchers later followed their idea and explored radiation induced polymerization processes, which was summarized by Chapiro. ${ }^{5}$ Although the exact nature of the reaction mechanism in radiation polymerization cannot be predicted a priori, the results obtained from radiation research were similar to those initiated by chemical means. Chain propagation in polymer systems was initially believed to be a free radical process, though it was later discovered that the mechanism varies from one chemical species to the other. Depending on the polymerizing media and synthesis conditions, chain reaction events followed a free radical or an ionic pathway and in some cases, a combination of both. The contributions of free radical mechanisms were investigated taking different criteria into account: ${ }^{4-7}$ action of free radical inhibitors, copolymerization, temperature effects and kinetic analysis. Most of the homogeneous radiation initiated reactive systems of styrene, methyl methacrylate and vinyl acetate polymerized by free radical mechanism. Free radical polymerization reaction rates should be directly proportional to the square root of the dose rate while the exponent was slightly higher in ionic polymerizations. Similar is the case with reactive systems in reactive precipitation systems, in which the exponent was predictably higher than 0.5 , following the results of chemical initiation in heterogeneous media. ${ }^{8}$ 
The evidence of ionic propagation in radiation polymerization was first observed when liquid isobutylene was $\gamma$-ray polymerized at low temperatures. ${ }^{9}$ This was supported by the $\gamma$-ray polymerization of butadiene and styrene in chlorinated solvents at room temperature. ${ }^{10}$ Based on these results, ionic polymerization was proposed to be the dominant pathway in low temperature radiation polymerization reactions. The effect of dose rate was also eventually determined from $\gamma$-ray polymerization of styrene at room temperature, both in chlorinated solvents and in bulk. In fact, both free radical and ionic mechanisms contributed to the propagation of chain reaction in the polymerization of styrene at room temperature, leading to a complex kinetic scheme. Many independent researchers published similar results on the $\gamma$-ray polymerization of vinyl monomers, at high dose rates. ${ }^{11-13}$ Westlake and his coworkers ${ }^{11}$ considered the contribution of both free radical and cationic propagation mechanisms while devising a kinetic scheme to simulate their experimental findings, and later validated their model with their experiments on $\gamma$-ray polymerization over a range of temperatures from $0^{\circ} \mathrm{C}$ to $-30^{\circ} \mathrm{C}$. Without introducing any simplifying assumptions, their model predicted the experimental results at a $90 \%$ confidence interval. The model predictions of G-values for styrene (0.69) also matched with those given by Chapiro. 5

\section{Radiation Polymerization in Precipitating Systems}

Most of the kinetic schemes and experimental observations referred to in the literature focused on radiation-induced solution polymerization. Kinetics of precipitating systems are much more complex than their homogeneous counterparts, due to the thermodynamic influence on the reaction events. Consequently, the effect of any parameter on the polymerizing system behavior is not as simple as it is in case of free radical reactions in homogeneous media. 
Radiation induced polymerization in precipitating media is not in vogue until recent times. Some of the precipitating systems that were discussed in literature ${ }^{19-21}$ are acrylonitrile, vinylchloride, styrene in 'poor solvents' such as alcohols. Radiation polymerization of acrylonitrile ${ }^{14}$ resulted in a fine powder of polymer particles, which essentially are macromolecular precipitants. Most of the polymer particles were reported to be of an average diameter of $1000 \AA$, or $100 \mathrm{~nm}$. This is due to the minimized chain mobility due to phase-separation in precipitating systems, which was also demonstrated as a part of the current study [Chapter 2]. The conversion profiles of $\gamma$-ray polymerization of acrylonitrile were also steady during the initial stages of the reaction, after which a linear increase was observed. The critical conversion in such systems was typically in the range of $1-3 \%{ }^{15,16}$ The effect of dose rate on the reactive precipitation systems depends on the $G_{R}$ values of monomer, solvent and polymer. Just as in chemical initiation systems, radiation-induced precipitating polymerization systems also showed dependency of reaction rates on dose rate with an exponent of $0.6-0.8$. The G-values of polymer, therefore, play an important role in the radiation polymerization of precipitating systems, especially at high conversions. Nevertheless, due to the low incident flux, gamma ray polymerization takes a long time with after-effects in the product being dominant even after several hours. For example, in $\gamma$-ray polymerization of acrylonitrile, after-effects were reported to be dominant until around $300-400 \mathrm{hrs}$, resulting in a $6 \%$ increase in conversion. $^{21}$

Despite the kinetic complexities, precipitating polymeric systems pose severe complications when a monomer is polymerized in a mixture of solvents and the polymer selectively absorbs one of the solvents. Polymerization of styrene in the presence of various precipitating solvents using gamma radiation was studied extensively by 
Chapiro et al. ${ }^{1}$ From the reaction rate data gathered as a function of polymeric mole fraction, it was concluded that for most of the systems, the linear energy transfer (LET) during the radiation polymerization of styrene is dominant in $\mathrm{n}$-heptane solutions, while cyclohexane, acetone and ether had little or no impact with respect to LET. The most important result from those experiments, however, was that the extent of energy transfer to the solvent in polymer-solvent systems can be related to the $\mathrm{G}$ values of monomers and solvents. Analogous to reactivity ratios, the extent of precipitation in an irradiated mixture depends on the ratio of G-values of respective components. In all the systems studied, a reaction rates increased gradually up to a limiting conversion of $50 \%$, after which the reaction rate suddenly dropped, which was attributed to the trapping of propagating radicals in polymer precipitates.

To summarize the discussion, high energy radiation in the form of $x$-rays, gamma-rays or electron beam, is an excellent alternative to the conventional chemical initiators. Unlike random chemical initiation, radiation initiation offers uniform initiation rates through out the polymerizing medium. Reaction rates in radiation induced polymerizing systems can be varied by choosing a spectrum of wavelengths for incident radiation and is also dependent on the reaction medium and synthesis conditions. These advantages not withstanding, high energy radiation, so far, was used only for the modification of polymer properties. Gamma-rays were traditionally used as an initiating source for polymerization reactions, while electron beam irradiation mainly focused on modifying the polymer properties by crosslinking. X-rays never figured in the scheme of radiation initiation sources mainly because of the lack of readily accessible high-flux, high energy sources. With the advent of high-flux radiation from synchrotron sources, $\mathrm{x}$-rays can be used as an initiating medium whose initiation rates can be easily controlled by using the 
required optical elements in the path of the beam. Hard x-rays from synchrotron can also be used in a lithographically defined exposure mode, to initiate polymerization in inverse-precipitation regions to synthesize polymeric microstructures in a "bottom-up" procedure.

\section{Introduction}

Polymer hydrogels have been shown to be biocompatible due to their ability to sorb large amounts of water. ${ }^{17}$ They are usually available in the form of dense or porous materials. Normally, hydrogels are molecularly crosslinked through covalent or ionic bonds. However, some thermoplastic hydrogels have also been recently developed. They are also classified in terms of their solubility or swelling characteristics. In particular, the thermoreversible hydrogel exhibits decreased (increased) solubility or swelling in water as the temperature is increased (decreased), due to phase transformation at the lower critical solution temperature (LCST).

Applications as thermoreversible gels have been reported for hydrogels and hydroplastics. ${ }^{18}$ Thermosensitive hydrogels are biodegradable in water and can be used in controlled drug delivery, controlled release, recovery, and extractions applications. ${ }^{19} \mathrm{~A}$ variation of the basic membrane formation process ${ }^{20}$ involves the formation of the phaseseparated gel (based on cellulose ether in water) above the LCST in a formulation that includes crosslinking agents. ${ }^{21}$ The resulting material was used as a microporous, fastresponse gel. The response rates could be further increased by introducing macropores into the hydrogel. ${ }^{22-24}$

Patterned thermoreversible hydrogels are a new class of materials that could have important utility in biomedical, pharmaceutical, and bioseparations applications. With 
patterning, especially in conjunction with micro- and nanofabrication, thermoreversible hydrogels could be used in microfluidic applications ${ }^{25}$ and other biocompatible nanotechnological systems. ${ }^{26}$

Most of the polymerizing systems initiated by $\mathrm{x}$-rays or $\gamma$-rays are reported to have formed free-radical species at high temperatures, with a few systems forming ionic species depending upon the operating conditions and the solvents used. ${ }^{1,9-13}$ After radiation-based initiation of a system that is above the LCST of the growing polymer, control over the propagation of the polymer chains is expected to result in the confinement of the polymer domains within the exposed area (following an FRRPP mechanism). Use of hard x-rays from a synchrotron radiation source facilitates the formation of high aspect-ratio structures of the polymer. Since the x-ray source can produce relatively small wavelength radiation $(\sim 1 \mathrm{~nm})$ at very high intensities, fine structures can be produced.

The effect of mixing on the reaction propagation and termination in FRRPP systems was demonstrated in a recent paper. ${ }^{27}$ Recalling that work briefly, mixing was shown to be the driving force behind inverse precipitation and globule-globule agglomeration in the polymerization of styrene in ether and a mixed solvent of tert-butyl acetate/water. In a quiescent fluid, in the absence of mixing, mass transfer in reactive FRRPP systems is translational-diffusion controlled. Therefore, in the unavailability of monomer, agglomeration of globules is possible only if the growing reactive domains actually interact with each other. Thus, the reactive domains in a quiescent system would have to grow and overlap each other in order to agglomerate. Unlike from a well-mixed reactor, it was therefore possible to observe nanoparticles from a quiescent fluid system, even after one half-life of the initiator. 
Radiation-initiated studies were also conducted in a quiescent fluid system in order to minimize the interaction between growing polymer domains. In the absence of chemical mediators, ionizing radiation can be used to selectively initiate specific regions and thus spatially control the reaction. In a chemical initiation system, distributed heat from the reaction exothern results in little rise in local temperature. On the other hand, in radiation-induced polymerization above the LCST, monomers are initiated uniformly through out the exposed regions resulting in high termination rates (by combination, due to equal radical reactivities) and thus low propagation rates (due to precipitation aided by exothermic reaction) to unexposed regions. Therefore, reaction control in radiationinitiated retrograde precipitating systems is aided by the continuous initiation inasmuch as it is by the exothermic reaction. Since reaction control in FRRPP mainly depends on the local thermal gradients, radiation-induced polymerization systems above the LCST offer better control over polymerization reaction, apart from the possibility of selective initiation for patterning.

\section{Experimental}

\section{Polymerization in a Quiescent-Fluid Reactor}

$\mathrm{N}$-isopropylacrylamide (NIPAm) was polymerized in a $1 / 16^{\prime \prime}-\mathrm{OD}$ tube reactor, which is assumed to maintain a quiescent fluid. A $0.08 \mathrm{~g}$ of monomer mixture along with 0.3 mg of VA-044 was dissolved per $1 \mathrm{ml}$ of water. A crosslinker, ethylene glycol dimethacrylate (EGDMA), was added at $10 \%$ (wt./wt.) to the monomer. The reactive mixture was degassed by bubbling nitrogen through it for about 15 minutes. The tube reactor was tightly sealed on one end, while the other end was connected to an Eldex metering pump. Reactive mixture was pumped into the tube reactor assembly for 5 
minutes at a rate of $1 \mathrm{ml} / \mathrm{min}$. The other end of the reactor was then sealed and the reactor assembly was immersed in a hot water bath, maintained at $45^{\circ} \mathrm{C}$. After 20 minutes, product was pumped out with water at $45^{\circ} \mathrm{C}$. The product was collected on a silicon wafer at liquid nitrogen temperature $\left(\sim-200^{\circ} \mathrm{C}\right)$, which was later freeze-dried in a mixture of xylenes and liquid nitrogen slush at $-40^{\circ} \mathrm{C}$. The freeze-dried product was then analyzed using an atomic force microscope (AFM).

\section{Synchrotron-Radiation-Induced Polymerization}

A mixture containing 20 wt $\%$ NIPAm, 2 wt $\%$ calcium methacrylate, and 78 wt $\%$ water was used for preliminary patterned polymerization work. Calcium methacrylate, which was produced by neutralizing methacrylic acid with calcium hydroxide, acts as a crosslinking agent. The mixture of monomers was thoroughly degassed to prevent recombination of radical species with dissolved gases in solvent.

The reactive fluid was sandwiched between two $4 "$ silicon wafers, one of which had an oxidized surface used to promote adhesion of the fluid and thus the polymerized pattern. The sides of the sandwich of wafers and reactive fluid were sealed with a Kapton ${ }^{\mathrm{TM}}$ tape. Spatially controlled radiation initiation studies were carried out at the SRI-CAT beamline $2-\mathrm{BM}-\mathrm{B}^{28}$ of the Advanced Photon Source (APS). The sandwiched wafer system was mounted onto a thermally controlled aluminum backing plate, maintained at $50^{\circ} \mathrm{C}$, in the path of the x-ray beam. This temperature is clearly above the LCST of poly (NIPAm) in water. ${ }^{29}$ Exposure was carried out through a gold mask to spatially control the exposures (Figure4.1). 


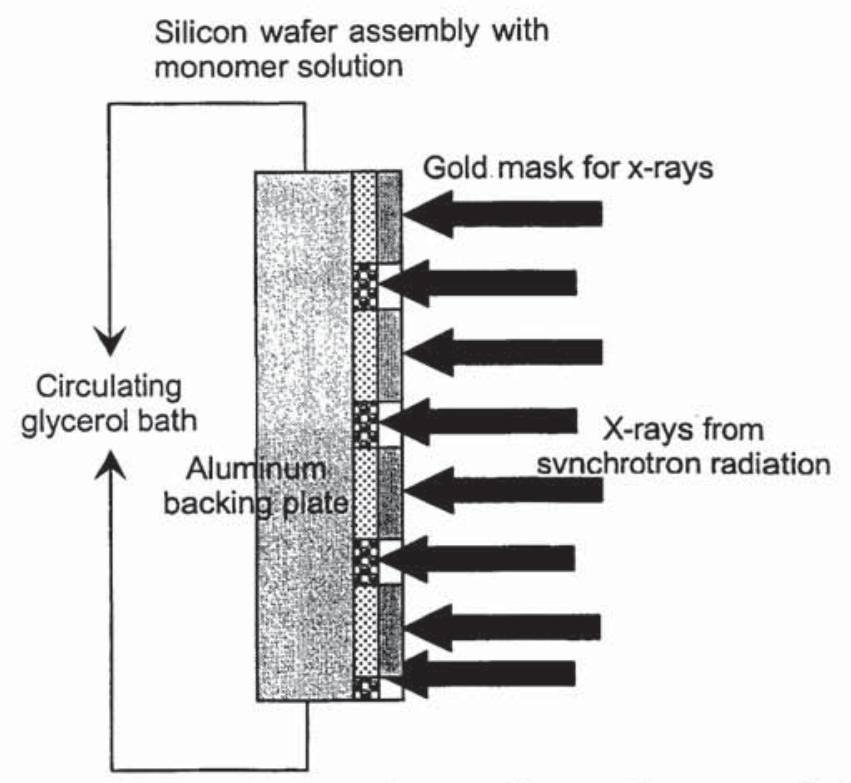

Figure 4.1: Schematic of an experimental setup for synchrotron-radiation-induced, spatially controlled polymerization.

After an exposure time of about 20 minutes (dosage $\sim 500$ a.u.), a hold time of 30 minutes was employed to allow the reaction to continue at $50^{\circ} \mathrm{C}$. The reaction was then frozen by exposing the sandwiched wafer system to dry ice. The wafer system was dismantled in dry ice, and the polymerized film was washed with water and vacuum dried in a desiccator. The dry pattern obtained was examined by optical microscopy and was profiled using a depth profilometer.

\section{Results and Discussion}

\section{Quiescent Fluid Polymerization}

Figure 4.2 shows AFM images of the freeze-dried poly (NIPAM-co-EGDMA) nanoparticles. Particle analysis of the images (not presented here; using Nanoscope IIIa software from Digital Instruments Inc.) indicates the persistence of nanopolymer 
particles with a mean radius of gyration of $30-35 \mathrm{~nm}$, characteristic of our quiescent fluid polymerization experiments. Styrene systems of our previous quiescent polymerization studies resulted in nanoparticles with no agglomeration. ${ }^{27}$ However, in the case of NIPAm, some agglomeration is observed, which might be an after-effect induced during the freeze-drying of aqueous solution.

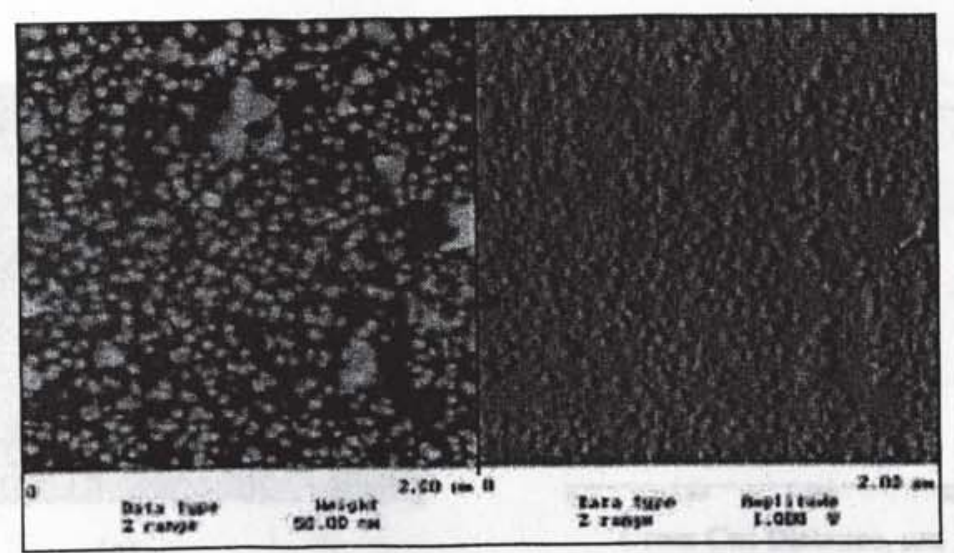

Figure 4.2: AFM topography scan of freeze-dried nanoparticles obtained from NIPAM-EGDMA polymerization in water. The left frame is based on height, while the right frame is based on amplitude.

When the freeze-drying temperature is relatively low, water could form crystallites encompassing the dispersed nanoparticles. During sublimation from the crystallite form, water evaporates leaving slightly agglomerated particles behind. In conventional polymerizing systems, NIPAm is polymerized below the LCST causing gelation to set in immediately, forming a bulk film. By polymerizing NIPAm in a retrograde precipitating environment, gelation can be avoided to produce isolated globules in space. Unlike conventional vacuum drying, freeze-drying helps in preserving these isolated globules. Because these nanoparticles are precipitating from the solution, the internal structure was suggested to be porous when swollen. ${ }^{30}$ Synthesis of poly (NIPAm) above the LCST also 
opposes the formation of surface skin, a phenomenon characteristic of conventional hydrogels, making these materials useful for in situ and in vivo bio-applications.

\section{Synchrotron-Radiation-Induced Polymerization}

Figure 4.3a shows the pattern of poly ( $\mathrm{N}$-isopropylacrylamide-co-calcium methacrylate) that was produced using a synchrotron radiation source. The polymer is

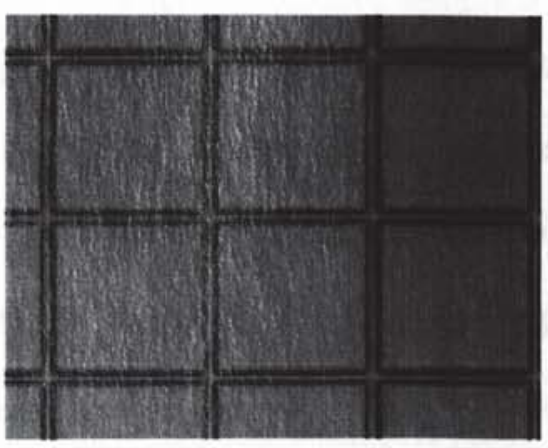

(a)

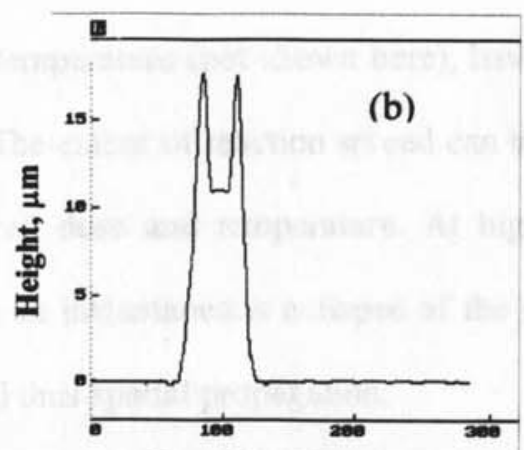

Cross Cut Distance, um

Figure 4.3. Right image shows an optical micrograph (5x) of the $25 \mu \mathrm{m}$ grid pattern, polymerized from NIPAm, at $50^{\circ} \mathrm{C}$. The distance between the lines is $500 \mu \mathrm{m}$. Surface profile of the grid lines in Figure 6, indicating a $14 \mu \mathrm{m}$ central plateau that is between two outlying peaks separated by $27 \mu \mathrm{m}$.

the material that makes up the lines of the square grid pattern, and it was produced by spatially controlling the exposure of the monomer mixture using a patterned 50 - $\mu$ m-thick gold mask, microfabricated via photolithography and gold electroplating. ${ }^{31}$ The mask was made up of $500 \mu \mathrm{m}$ squares of gold that are separated by a width of $25 \mu \mathrm{m}$. A closer examination of the lines (Figure 4.3b) indicates that the edges are raised relative to its interior.

The distance between the peaks is $27 \mu \mathrm{m}$, while the horizontal distance at the base is $59 \mu \mathrm{m}$. The central plateau region in the interior has a horizontal distance of $14 \mu \mathrm{m}$, 
which could be closer to $25 \mu \mathrm{m}$ in wet state. The twin peaks in the profiles are uniformly present along the edges of the lines in Figure 4.3a, showing that there was controlled reaction outside the beam cross-section of $25 \mu \mathrm{m}$, which tends to form a less crosslinked material (polymer-lean phase) than what was produced within the beam cross section (polymer-rich phase). Therefore, it is clear that the polymer front did not extend beyond $20 \mu \mathrm{m}$ or so from the exposed regions, and the intended square pattern was produced. The patterns produced, at the same dose and temperature (not shown here), have similar reaction spread, around the exposed regions. The extent of reaction spread can be further minimized depending on the dose rate, overall dose and temperature. At higher dose rates, the reaction should be faster leading to an instantaneous collapse of the polymerrich phase, locking in the domain structure and thus spatial propagation.

\section{Conclusions:}

Poly ( $\mathrm{N}$-isopropylacrylamide) microstructures were synthesized from an $\mathrm{x}$-ray-initiated controlled free-radical polymerization process. The concept of reaction control in chemically-initiated retrograde precipitation systems has been verified in quiescent fluid reactor systems by the synthesis of Poly (NIPAm) nanoparticles. Since these hydrogels are prepared in a hydrophobic environment, they should be capable of higher swelling/deswelling and reswelling rates in water compared to those of conventional hydrogels. The structures are also of high purity due to the absence of chemical mediators, making them attractive for various biological, pharmaceutical, and microfluidic applications. Since the reaction pathway does not depend on the method of initiation, the observed reaction control in chemical-initiation systems can also be attributed to radiation-initiation systems. By combining with a top-down lithographic 
approach, this synthesis method can be used to produce polymer microstructures from systems which possess a LCST. Work is currently in progress to study the process and optimize the parameters controlling the reaction. 


\section{References}

1. Chapiro, A.; Radiation Chemistry of Polymeric Systems, Interscience Publishers, Great Britain, UK, 1962.

2. Burton, M., Disc. Faraday Soc., 1952, 12, 317.

3. Hopwood, F. L., Phillips, J. T., Nature, 1939, 143, 640.

4. Lander, Y.; Thesis, University of Paris, 1952.

5. Chapiro, A.; J. Chem. Phy., 1950, 47, 747.

6. Schmitz, J. V., Lawton, E. J., Science, 1951, 113, 718.

7. Burlant, W. J., Green, D. H., J. Polym. Sci., 1958, 31, 227.

8. Abere, J., Goldfinger, G., Naidus, H., Mark, H.; J. Phy. Chem., 1945, 49, 211.

9. Grosmangin, J., Magat, M., Chem. Commun., 1957, 22, 141.

10. Burnett, G., M.; Mechanisms of Polymerization Reactions, Interscience Publishers, Inc., NY, 1954.

11. Westlake, J. F., Huang, R., J. Polym. Sci. - Part A1, 1972, 10, 1429, 2149.

12. Squire, D. R., Cleaveland, J. A., Hossain, J. M. A., Oraby, W., Stahel, E. P., Stannett, V. T., J. Appl. Polym. Sci., 1972, 16, 645.

13. Moore, P. W., Clouston, J. G., Chaplin, R. P., J. Polym. Sci.: Polym. Chem. Ed., 1983, 21, 2491. 
14. Thomas, W. M., Thomas, A.M., Deichart, W. G., Proc. Intl. Symp. Macromol. Chem., Weisbaden, 1959.

15. Bensasson, R., Prevot - Bernas, A., J. Polym. Sci., 1958, 30, 163.

16. Bernas, H., Bodard, M., J. Polym. Sci., 1960, 48, 167.

17. Park, H. and Park, K., "Hydrogels in Bioapplications," in: Hydrogels and Biodegradable Polymers for Bioapplications, R.M. Ottenbrite, J. Huang, and K. Park, Eds., ACS Symposium Series, 1996, 627, Chap. 1.

18. Mueller, K.F., "Thermotropic biphilic Hydrogels and Hydroplastics," U.S. Patent No. 5,104,954, 1992, April 14.

19. Wu, C. and Jiang, S., "Polymer Gel Composition and Uses therefor," U.S. Patent No. 6,030,634, 2000, February 29.

20. Tkacik, G., "Porous Polymeric Structures and a Method of making such Structures by means of heat-induced Phase Separation," U.S. Patent No. $5,444,097,1995$, August 22.

21. Kabra, B.G. and Gehrke, S.H., "Microporous fast response Gels and Methods of Use," U.S. Patent No. 6,030,442, 2000, February 29.

22. Hirasa, O., Ito, S., Yamauchi, A., Fujishige, S., and Ichijo, H., Polymer Gels, Ed. DeRossi, D. et al., Plenum, New York, NY, 1991.

23. Kabra, B. G. and Gehrke, S. H., Polym. Commun., 1991, 32, 322. 
24. Wu, X. S., Hoffman, A. S., and Yager, P., J. Polym. Sci.: Part A, 1992, 30, 2121.

25. Studt, T., R\&D Magazine, 1995, June Issue, 22.

26. Drexler, K.E., Nanosystems: Molecular Machinery, Manufacturing, and Computation, John Wiley and Sons, New York, NY., 1992.

27. Tirumala, V. R., Caneba, G. T., Dar, Y. L., Wang, H. H., and Mancini, D. C., Adv. Polym. Tech, 2003, 22, 2, 126.

28. Lai, B., Mancini, D. C., Yun, W., and Gluskin, E., Proc. SPIE, 1996, 2880, 171.

29. Brandrup, J., Immergut, E. H., and Grulke, E. A., Polymer Handbook, $4^{\text {th }}$ Ed., John Wiley and Sons, New York NY., 1989.

30. Walther, D. H., Blanch, H. W., and Prausnitz, J. M., LBL Report No. 34875, UC-401, Lawrence Berkeley Laboratory, Berkeley, CA, 1993.

31. Mancini, D. C., Moldovan, N. A., Divan, R., De Carlo, F., and Yaeger, J., Proc. SPIE, 2001, 4557, 77. 


\section{Chapter 5}

\section{A Novel Spatially-Controlled Synthesis of Poly (N-Isopropylacrylamide) and Poly (Methacrylic Acid) Microgels}

\section{Introduction}

Radiation synthesis of polymeric materials for several biomedical and biochemical applications was extensively researched by many groups in the past. ${ }^{1-4}$ Gamma-rays from a lab source $\left({ }^{60} \mathrm{Co}\right)$ typically serve as an irradiating medium for flood exposures that result in bulk polymers with no spatial definition. Irradiation of polymers with an electron beam is also a common practice in sterilization of materials for medicinal and space applications. ${ }^{5,6}$ High energy electron beams from linear accelerators is routinely used in pulsed radiolysis techniques to extract complex chemical kinetics during polymerizàtion reactions. ${ }^{7-9}$ Synchrotron radiation, whose characteristics are quite similar to those of other accelerator sources, was largely overlooked by the polymeric and biosynthesis community although it has been heavily subscribed as a source for $\mathrm{x}$-ray lithography ${ }^{10}$ to produce deep $(\sim 1 \mathrm{~mm})$ and ultra deep $(\sim 1 \mathrm{~cm})$ microstructures for subsequent developing and metal electroplating of LIGA-based microelectromechanical 
components. Initial forays into synchrotron-radiation-induced chemistry were also more focused towards surface modification and metal nanoparticle deposition and growth induced by synchrotron radiation but did not concern with synthesis of soft matter. ${ }^{11-15}$ Sclavi et al recently extended the concept of synchrotron radiation-induced scissioning of polymers to study the protein folding rates in precipitating media. ${ }^{16}$ Using radiation damage of DNA as a "footprint" to track the folding steps, their group studied the kinetics of folding issues at millisecond time resolutions.

Conventional synthesis of hydrogels, which can be tailored to respond to a variety of external stimuli, is typically carried out by chemical initiation methods that produce bulk, macroscopic gels. As is the norm with polymers, conformation of gels to any size or shape is later achieved by means of casting or molding. Spatially well-defined gel structures that can respond to external stimulus are, therefore, much needed for potential applications in controlled release and pharmaceutics. Recent developments in fabrication of microgel structures employed the use of photopolymerization and stereolithography to produce two and three-dimensional patterns ${ }^{17-19}$ for their use as microfluidic valves, in situ sensors and bioactive materials. However, the use of ultraviolet (UV) radiation in such methods impaired pattern accuracy, specifically at smaller line widths $(<300 \mu) .{ }^{19}$ Use of synchrotron radiation, with a premeditated energy spectrum to initiate polymerization, surpasses such limitations of UV-based chemistry like photolithography and photopolymerization while offering a uniform reaction rate even in ultra-deep monomer solutions. It would, therefore, be desirable to invoke a reaction chemistry that would limit polymerization propagation to the irradiation zones, with reasonable accuracy. The ability to be able to graft copolymerize patterns of different monomers during the synchrotron radiation-induced synthesis renders this technique a unique 
advantage of combining multiple-step polymer microfabrication procedures into a singlestep synthesis.

\section{Polymerization in Inverse Precipitation Systems}

Precipitation polymerization above the lower critical solution temperature (LCST) is also otherwise called as the free radical retrograde precipitation polymerization. ${ }^{20}$ Conventional reactive precipitation systems are marred by the fact that the exothermic reaction continuously drives the polymerization reaction away from the phase envelope and into the solution, thus offering little control over the propagation and termination reaction which define the product properties like molecular weight, polydispersity etc. On the contrary, the exothermic polymerization chain reaction in inverse precipitation regime drives the growing polymer chains deeper into the phase envelope (coil-toglobule transition). ${ }^{21}$ Further diffusion of monomer into the collapsed polymer chains is prevented by the uphill thermal diffusion barrier existing due to the ongoing exothermic reaction within the precipitated globule. The chain propagation and termination rates in the phase-separated polymer globules will eventually be severely suppressed due to the unavailability of the monomer. ${ }^{22-24}$ Consequently, mixing plays an important role in controlling the reaction kinetics of this exotherm-driven precipitation polymerization.

Thermal equilibrium, caused by mixing, promotes inverse precipitation uniformly through out the reactive system. In the absence of it, the pockets of precipitated growing polymer globules, whose interior is proposed to be at a higher temperature due to the continuous exothermic chain reaction (hot spots), are driven towards higher reaction rates producing isolated polymer nanoparticles from increased chain collapse at higher 
temperatures, while the rest of the medium remains as a solvent-rich environment. This hypothesis was experimentally verified by carrying out polymerization in a quiescent reactive system, which preserves the "hot spots" due to thermal inequilibrium, leading to the formation of nanopolymer precipitates. ${ }^{25} \mathrm{We}$ have recently applied this process to the synthesis of hydrogels, in which we have shown that the reaction propagation and termination in inverse precipitation systems can be carefully controlled to produce polymeric nanoparticles. Such phase-separated hydrogels should be characteristic of fast response as observed by Kabra and Gehrke. ${ }^{26}$ Extending this concept of spatiallyisolating the reaction zones of a quiescent reactive system, we proposed that polymeric microstructures can also be synthesized, not necessarily microfabricated from a prepolymer, by a novel $x$-ray induced polymerization chemistry above the LCST [Chapter 4].

\section{Experimental}

\section{Materials Used}

Methacrylic acid (MAA) and N, N'-Methylene bisacrylamide (MeBAm) were purchased from Aldrich Chemical Company. MAA was double-distilled before use, while MeBAm was used as-is. N-isopropylacrylamide (NIPAm) (>99\%) and poly (ethyTene glycol) (PEG-600), with an average molecular weight of 600Da, were obtained from Arcos Organics Ltd. and were used as-is. A small amount of benzoquinone present in NIPAm as an inhibitor was ignored since the incident radiation was energetic enough to initiate the reaction, despite its presence. Unconjugated gold colloids, with an average diameter of $5 \mathrm{~nm}$, were purchased from British BioCell International limited. 
These gold sols are not tagged with any biological or organic component and exist in a relatively unagglomerated state in water. Unless otherwise stated, the water was deionized before use.

\section{Sample Preparation}

Monomer solutions were prepared by dissolving monomers in water on a weight-byweight basis. An in-house sample holder made from silicon wafers separated by polyimide spacers, was used for holding monomer solutions. The wafers were sealed along their circumference with a Teflon threadseal tape for leak-free seal and then a Kapton glue tape. Solutions were injected into the wafer assembly after partially sealing the circumference (Figure 5.1), and exposed to bending magnet radiation from a synchrotron.
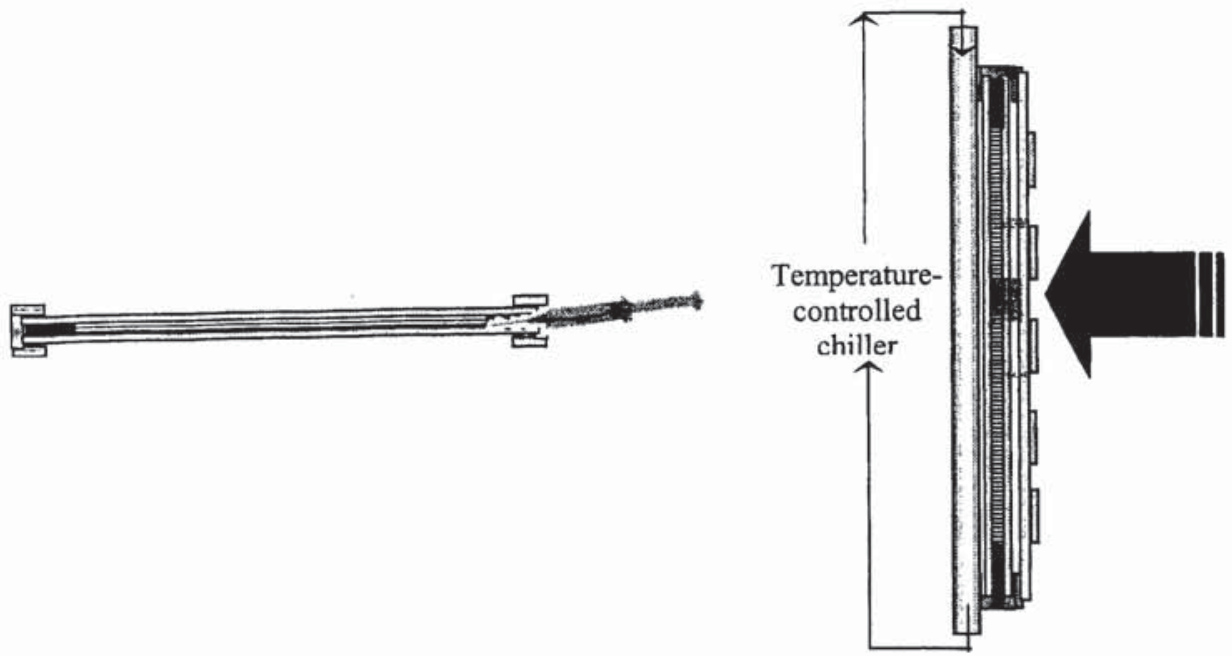

Figure 5.1; Schematic of the liquid-containment system (left), and its exposure set up on the beamline, 2-BM.

\section{Spatially-Controlled Polymerization}

Radiation-induced polymerization studies were carried out at the bending magnet beamline, 2-BM-B of SRI-CAT, at the Advanced Photon Source. The beamline was 
highly optimized to run deep x-ray lithographic exposures of PMMA, in a "pink" beam configuration that provides a photon fluence of approximately $10^{12}$ photons $/ \mathrm{sec} / \mathrm{mm}^{2}$, with critical photon energy at $19.5 \mathrm{keV}$. Energy profile of the beamline and its transmission and absorption characteristics through the various optical elements in the path of the beam and in monomer solutions are discussed elsewhere [Chapter 7]. Power absorbed in the $20 \%$ monomer solutions, used in these experiments, was calculated as a difference in the power of incident radiation at the surface of the solutions and that of transmitted past the solutions of any given thickness*. X-ray induced polymerization was carried out by maintaining the monomer solutions at a reaction temperature by circulating a fluid from a temperature-controlled chiller through an aluminum backing plate that supports the wafer assembly. As shown in Figure 5.1, an x-ray mask, fabricated from photolithography and gold electroplating, was used to spatially shield specific regions of exposure. Masked irradiation, which is a common practice in lithography, provides a way to initiate the reaction in a premeditated spatially-defined manner that is unachievable in chemical initiation systems. Monomer solutions were polymerized by irradiating them to reach an absorbed radiation dose that is equivalent to a time of reaction. The wafer assembly was dismantled after a stipulated time of exposure and the samples were airdried in a fume hood to remove the solvent without any post-processing procedure.

\section{Microgel Characterization}

Microgel patterns obtained were characterized by optical microscopy using an upright Nikon microscope equipped with a digital camera.

* Energy losses due to incoherent scattering were not considered in these calculations. 


\section{Results and Discussion}

\section{Polymerization-Induced Self Assembly of Thermoreversible Microgels}

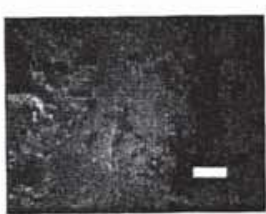

(a)

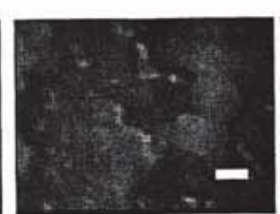

(b)

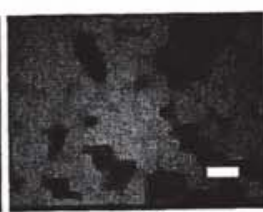

(c)

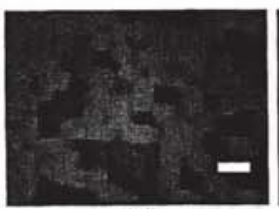

(d)

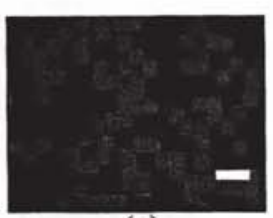

(e)

Figure 5.2: Evolution of poly (NIPAm) microgel patterns (a-e) obtained at various irradiation times (absorbed doses). (a) shows a poymer-lean gel formed irrespective of irradiation zones $\left(1.28 \mathrm{~J} / \mathrm{mm}^{3}\right)$. (b) shows the initial microphase-separation of polymerrich and polymer-lean phases $\left(1.93 \mathrm{~J} / \mathrm{mm}^{3}\right)$. (c), (d) \& (e) show the gradual phase separation of patterns and their alignment into irradiated zones with an increase in molecular weight $\left(2.57,3.2\right.$ and $48.2 \mathrm{~J} / \mathrm{mm}^{3}$ respectively $)$. Bar $=200$ microns.

Figure 5.2 shows the evolution of poly (NIPAm) microgel patterns with an increase in overall dose that corresponds to an increase in molecular weight. The gels shown in Figure 5.2 were all prepared from a $20 \%$ monomer solution. As is typical of reactive systems in inverse precipitation region, the growing polymer chains formed due to the irradiation of monomers do not phase-separate until a critical molecular weight is reached. Below the critical dose that corresponds to a critical molecular weight, the polymer-lean gel will remain agglomerated in the solvent-rich environment and do not retain the structure in which it is formed (Figures $5.2 \mathrm{a}, \mathrm{b}$ ). As the molecular weight increases with time of exposure, the growing polymer chains slowly start to phase separate out of the solvent into the irradiated zones. The excluded solvent then forms a solvent-rich ambience around the phase separated patterns. As seen from Figure $5.2 \mathrm{~b}$, molecular weight of gels irradiated for short exposure times will not be high enough to 
exclude the absorbed solvent but will form a weak gel, coexisting with the solvent. At high enough absorbed energies (high molecular weights) the microgel precipitates in the patterns of irradiated regions by excluding the solvent to form a polymer-rich phase (Figure 5.2c-e). This phenomenon is similar to the self-assembly process in polymerpolymer blends that possess a lower critical solution temperature (LCST) ${ }^{27,28}$ and in polymerization-induced phase separation of liquid crystals or block copolymers. ${ }^{29-31}$ Since the time of exposure also corresponds to the extent of phase separation, the absorbed dose influences the polymer morphology, which can only characterized by gelresponse.

Since this is a combination of "bottom-up" synthesis and "top-down" lithography, gels of any given shape can be synthesized (Figure 5.3) although the limitation on size arises from the smallest distinguishable mask feature, for the incident radiation. Patterns need not necessarily be synthesized in isolated locations from one another (Figure 5.2-e) but can also be structured in a polymer layer. For example, a polymer film with carefully designed holes of any shape and size (both micro- and macroscopic) can be synthesized from this process, by using a cylindrical post as a mask out the incident radiation (Figure 5.3b). It was observed that the radiation-induced polymerization in the retrograde-precipitation region can be spatially controlled even at $10 \mu$-spacings. The smallest feature-size replicated using hard $\mathrm{x}$-rays was about $8 \mu$, at an aspect-ratio of 8 . In principle, extrapolating from the study of controlled chemical synthesis to produce nanoparticles, the reaction should be spatially controllable even down to $100 \mathrm{~nm}$ by choosing a proper initiation source. The process should also be generally applicable to any monomer-polymer-solvent system that possesses an inverse 


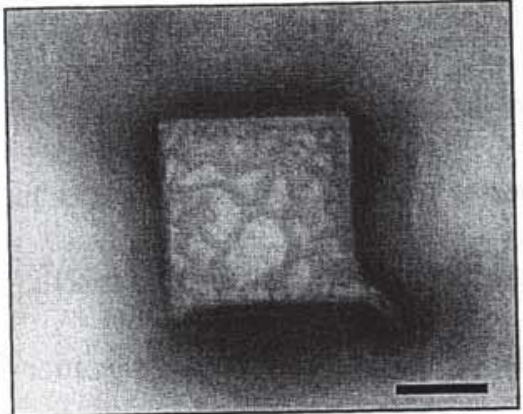

(a)

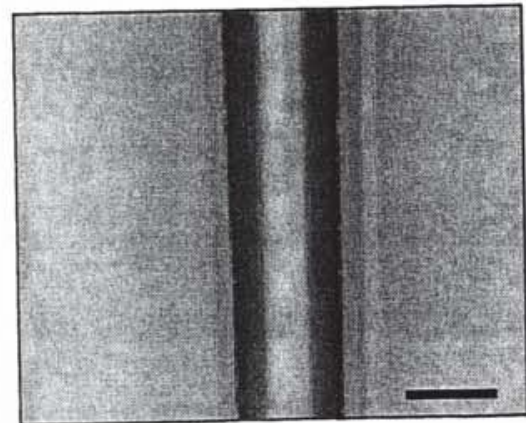

(c)

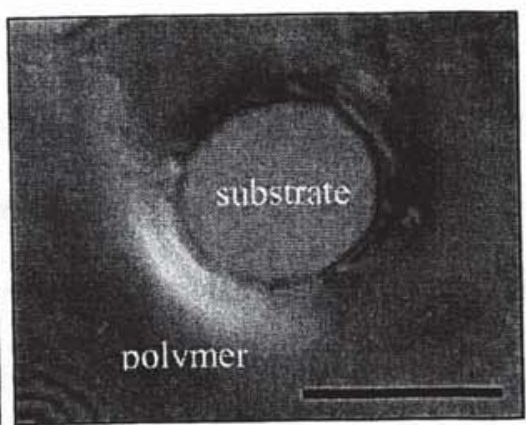

(b)

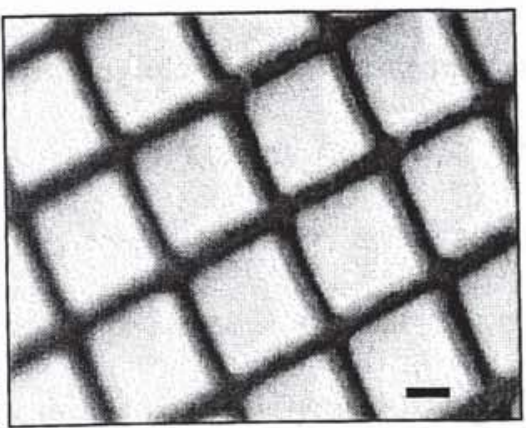

(d)

Figure 5.3: Thermoreversible microgel structures of various shapes and sizes prepared by $\mathrm{x}$-ray induced spatially-controlled polymerization of $\mathrm{N}$-isopropylacrylamide above the LCST. (a) A $100 \mu$-wide strain-gauge, with a $10 \mu$ extension needle. (b) PNIPAm film generated around a hole by employing a gold post of the same diameter as a mask. (c) Same as (b) but a $70 \mu$-wide rectangular block was used as a mask instead of a circular post. (d) Microgel blocks generated by exposing the monomers through a $100 \mu$-wide line grid. Bar $=50$ microns.

phase envelope, even if it is at high pressures. An increase in pressure is known to decrease the LCST of a precipitating system. Instead of the in-house wafer assembly used for irradiation systems at atmospheric pressure, a novel high pressure system can be designed for such environments. 
Synthesis of Ionic Microgels

To generalize the concept of radiation-induced spatial control in FRRPP systems, the current methodology was used to polymerize a well-studied methacrylic acid system. Microgel patterns based on methacrylic acid can likewise be self-assembled using the process mechanism described above, in which the physical features can be pre-defined while fabricating the mask. Polymerization of methacrylic acid above its LCST in water was earlier investigated by time-resolved small angle $\mathrm{x}$-ray scattering measurements at different temperatures [Chapter 3]. The pre-reacted system showed a linear chain collapse due to precipitation, while the chain growth profiles of reactive systems were more complex due to the associated chain reaction phenomena with the precipitation. The exact influence of kinetic and thermodynamic events on the polymer chain growth cannot be directly from these experiments. The growing polymer chains, nevertheless, reach limiting chain lengths even before two half-lives of the initiator, similar to the limiting conversions observed in the system [unpublished results].

Poly (methacrylic acid) microgels were prepared from hard $x$-ray induced polymerization of $20 \% \mathrm{MAA}$ in water. Note that the monomer concentration has little influence on pattern replication aspects and only effects the gel response to an external stimulus. Unlike PNIPAm, methacrylic acid forms a lean-gel through out the solution and does not exhibit good contrast between the exposed and unexposed zones (Figure 5.4a,b). However, this effect is only limited to low temperature radiation polymerization of MAA. A lean gel also coexists with the polymer-rich phase even at high temperatures, but quickly breaks apart due to the surface strain induced by severe chain collapse in the irradiated polymer, thereby offering excellent image contrast (Figure 5.4c, d). 


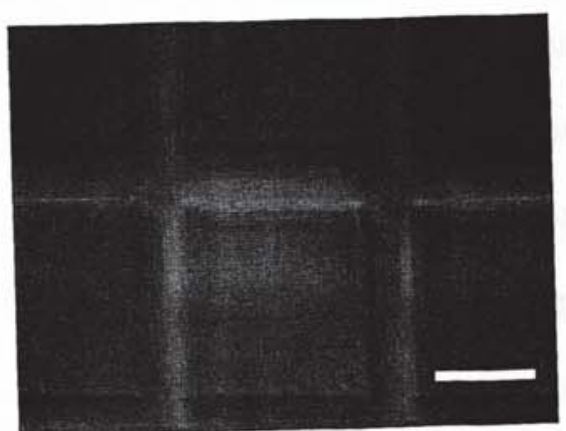

(a)

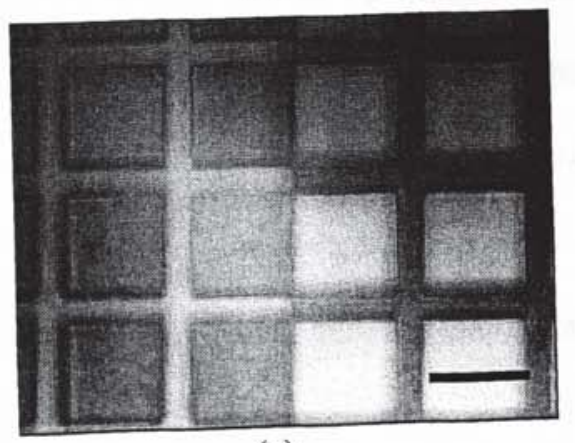

(c)

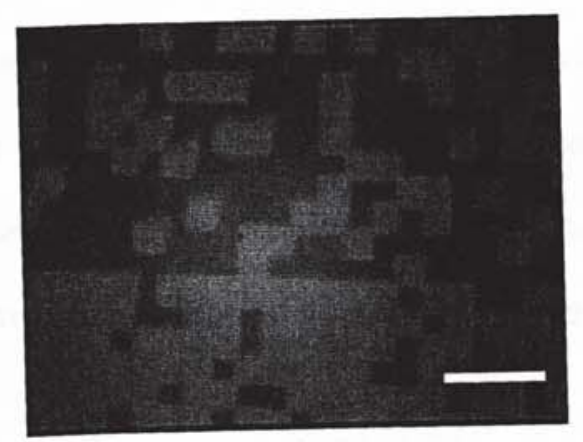

(b)

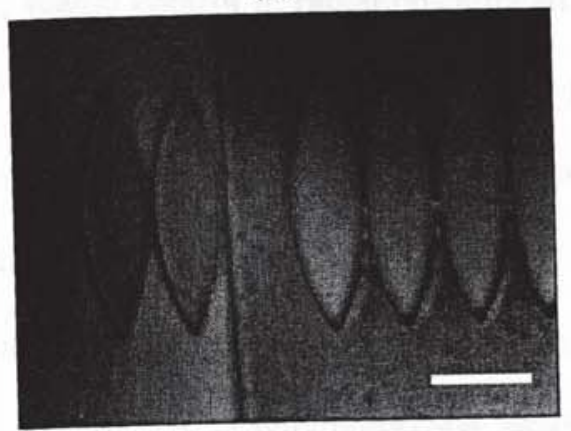

(d)

Figure 5.4: Ionic microgel structures prepared from radiation-induced spatiallycontrolled polymerization of methacrylic acid at $60^{\circ} \mathrm{C} \mathrm{(a)} \mathrm{\&} \mathrm{(b),} \mathrm{and} \mathrm{at} 80^{\circ} \mathrm{C} \mathrm{(c)} \mathrm{\&} \mathrm{(d).}$ All the patterns were imaged when immersed in water. (a) and (b) clearly show a diffused focus, originating from a lean-gel covering the pattern surface. (c) and (d) do not suffer from such artifacts since they were imaged through water and in the absence of any such polymer film. Bar $=400$ microns.

As is the case with chemically-initiated FRRPP systems, methacrylic acid initially forms a polymer-lean gel during the gel-effect phase of its conversion profile, after which precipitation starts to exercise control over the polymer chain growth. At low temperatures $\left(60^{\circ} \mathrm{C}\right)$, the gel-effects phase lasts longer than it does at high temperatures and the chain propagation rates in both the polymer-lean and polymer-phase will not be drastically different even after phase separation. Patterns from spatially-controlled methacrylic acid polymerization at $60^{\circ} \mathrm{C}$, therefore, coexist with a lean gel that is 
inseparable from the patterned precipitates. At higher temperatures $\left(80^{\circ} \mathrm{C}\right)$, on the other hand, reaction rates inside the precipitates will be much higher compared to those in the solvent phase. This leads to a faster increase in molecular weight and thus faster phaseseparation eventually resulting in an improved image contrast of replicated patterns.

\section{Synthesis of Nanohybrid Microstructures}

Summarizing the results presented above, spatial control in radiation-initiated FRRPP systems is inherently imparted by the phase equilibrium thermodynamics of the polymer and solvent. Since there are no external chemical mediators, presence of a neutral/reactive component, one that does not have an adverse affect on the polymerization, during reactive-precipitation coprecipitates that component along with the polymer. If the component is partially activated during polymerization, precipitation will also be driven by that constituent. For example, by conventionally polymerizing NIPAm in a gold nanoparticle solution, the PNIPAm precipitates would physically encompass the metal nanoparticles during phase-separation, though there would be no chemical association between the polymer and the metal. On the contrary, when NIPAm is radiation polymerized in a solution of gold nanoparticles, one can synthesize polymers that are covalently-linked to the metal nanoparticles at the core due to the higher radiation absorption characteristics of metal compared to an organic compound. Irradiated metals also generate more secondary electrons compared to their organic counterpakts. Due to the higher rates of absorption, gold nanoparticles, present in an otherwise normal FRRPP system, take up the role of creating nanoscopic "hot spots" in the precipitation environment, even before the growing chains are long enough to precipitate. The presence of secondary electrons should also help in covalently binding the gold sols to the growing polymer chains and in precipitating them simultaneously. 
Following this line of analysis, NIPAm was radiation polymerized in an unconjugated gold colloid solution. Gold nanoparticles of $5 \mathrm{~nm}$ diameter also emit a weakly-pink radiation when exposed to a mercury lamp. The swollen microgel patterns synthesized in this process were thus confirmed to contain the unconjugated gold sols from optical microscopy (Figure 5.5).
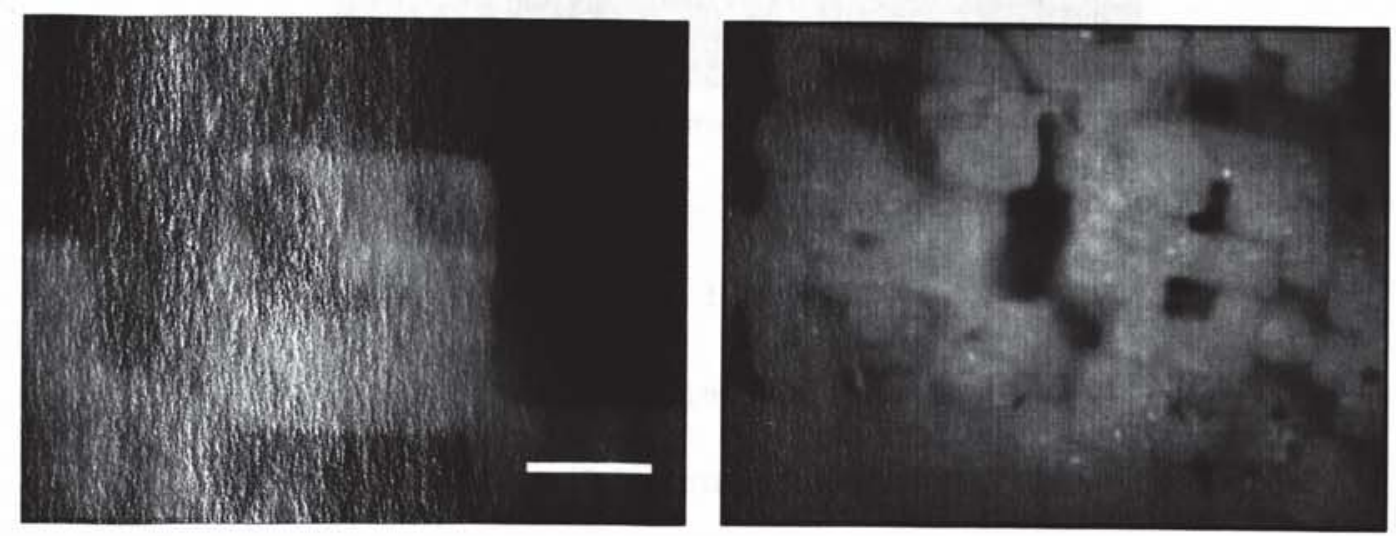

Figure 5.5: Poly (NIPAm-co-Au) microgels synthesized by coprecipitating unconjugated gold sols along with PNIPAm. Image to the left corresponds to patterned polymerization of NIPAm, above the LCST. Image to the right is a corresponding result but from polymerization at room temperature. $\mathrm{Bar}=100$ microns.

The concept of single-step nanohybrid synthesis can also be applied to copolymerization of a monomer mixture, in which both the monomers are soluble in the solvent and one of the product polymers is incompatible with the ambient solution. The phase-separated polymer patterns should consist of phase-separated macromolecular domains just as in case of a hydrophobic-hydrophilic copolymer. To verify this hypothesis, NIPAm was also polymerized in the presence of another low molecular weight polymer PEG-600 (Figure 5.6). Coincidentally, PEG has a phase transition in water at $16^{\circ} \mathrm{C}$. Morphological investigation into such gels by small angle scattering should, in principle, reveal three 


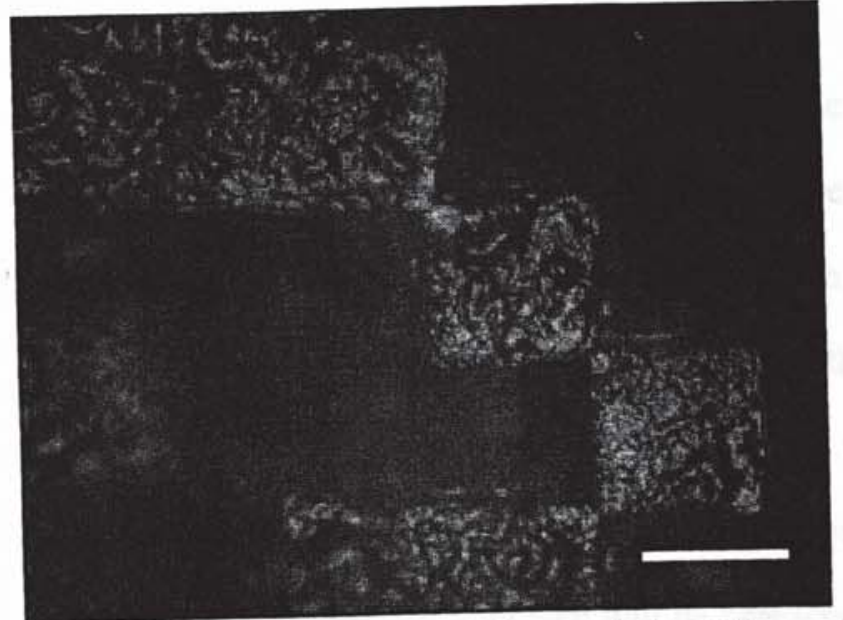

Figure 5.6: Poly (NIPAm-g-PEG600) microgels synthesized by graft polymerizing NIPAm in the presence of PEG- 600 , at $45^{\circ} \mathrm{C}$. Note the rich morphology of these gels compared to that of PNIPAm gels. Bar $=100$ microns.

morphologies: one below $16^{\circ} \mathrm{C}$, one above $16^{\circ} \mathrm{C}$ but below the LCST of PNIPAm $\left(32^{\circ} \mathrm{C}\right)$, and one more above $32^{\circ} \mathrm{C}$. Study of the temperature-induced morphological changes of these graft copolymer gels by small angle neutron scattering is currently in progress. The results from those experiments can be compared to similar small angle scattering investigation on the PNIPAm gels, which revealed the effect of dose, temperature and monomer concentration on the morphology of the gels [Chapter 6].

\section{Conclusions}

Conventional chemically-initiated bulk syntheses of hydrogels result in nanoporous gel network which offers resistance to the diffusion of water through the pores. Bulk gels are also slow to respond to an external trigger, mainly due to their increased volume in a swollen state. The volumetric transition rates can be increased by decreasing the size of gels. An increase in pore-size of the gel network also serves the same purpose of increasing the response rates of the gel. The synthesis route presented in this study 
produces microgels in a phase-separated state. Size of the microgels can be predefined by the mask features used during polymerization, while its pore-size can be controlled by choosing a proper quench depth, which is the difference between the reaction temperature and the critical solution temperature. Microgel structures synthesized by this procedure should also be ultra fast-responsive to external stimuli, and their response properties will be characterized at a later stage since it is beyond the scope of this study. Morphology of the gels prepared by the current approach will be characterized by small angle neutron scattering and is a part of our ongoing work.

It was shown that, irrespective of the monomer type, FRRPP systems can inherently control the spatial propagation of the polymerization reaction. An increase in reaction temperature thermodynamically favors an increase in image contrast of the replicated patterns. The total absorbed dose determines the pattern replication aspects up to a critical time of exposure, beyond which further irradiation leads to a change in gel morphology and their response to external stimulus. The synthesis strategy described in the current study is simple and also versatile, leading to nanocomposite microgel structures that can respond to multiple stimuli. 
Chapter 5: Novel Synthesis of Microgels

\section{References}

1. Hoffman, A. S., Gomboltz, W. R., Uenoyama, S., Radiat. Phy. Chem., 1986, 27, 275 .

2. Gao, D. Y., Yoshida, M., and Kaetsu, I., Eur. Polym. J., 1988, 24, 1037.

3. Kaetsu, I., Adv. Polym. Sci., 1993, 105, 81.

4. Kumakura, M., Kaetsu, I., Imunol. Commun., 1984, 13, 199.

5. Kinstle, J. F., In "Radiation Curing of Polymeric Materials", ACS Symposium Series, 417, Hoyle, C. E., Kinstle, J. F. Eds., 1990, 17.

6. Grossman, E., Gouzman, I., Nucl. Instrum. Methods Res., 2003, 208, 48.

7. Schneider, C., Swallow, A. J., J. Polym. Sci. Polym. Lett., 1966, 4, 277.

8. Metz, D. J., Potter, R. C., Thomas, J. K., J. Polym. Sci: A-1, 1968, 5, 877.

9. Tagawa, S., Katsumara, Y., Tabata, Y., Chem. Phy. Lett., 1979, 64, 258.

10. Lai, B., Mancini, D. C., Yun, W., Gluskin, E., Proc. SPIE, 1996, 2880, 171.

11. Rosenberg, R., Mancini, D. C., Nucl. Instrum.. Methods Phy. Res. Sect. A, 1990, $291,101$.

$\checkmark$

12. Rosenberg, R., Ma, Q., Farrell, W., Keefe, M., and Mancini, D. C., Rev. Sci.Instrum., 1997, 68, 2550.

13. Ma, Q., Mancini, D. C., Rosenberg, R., Appl. Phys. Lett., 1999, 75, 2274. 
14. Ma, Q., Moldovan, N., Mancini, D. C., Rosenberg, R., Appl. Phys. Lett., 2000, 76, 2014.

15. Ma, Q., Moldovan, N., Mancini, D. C., Rosenberg, R., Appl. Phys. Lett., 2002, $81,1741$.

16. Sclavi, B., Sullivan, M., Chance, M. R., Brenowitz, M., Woodson, S. A., Science, 1998, 279, 1940.

17. Beebe, D. J., Moore, J. S., Bauer, J. M., Qing, Y., Liu, R. H., Devadoss, C., Byung-Ho, J., Nature, 2000, 404, 588.

18. Hilt, J. Z., Gupta, A. K., Bashir, R., and Peppas, N. A., MRS Proc., 2003, in press.

19. Liu, V. A., Bhatia, S. N., Biomedical Devices, 2002, 4, 257.

20. Caneba, G. T., Adv. Polym. Tech., 1992, 11, 277.

21. Nishio, I., Swislow, G., Sun, S., Tanaka, T., Nature, 1982, 300, 243.

22. Aggarwal, A., Saxena, R., Wang, B., Caneba, G. T., J. App. Polym. Sci., 1996, 62, 2039.

23. Wang, B., Dar, Y. L., Shi, L., Caneba, G. T., J. Appl. Polym. Sci., 1999, 71, 61.

24. Dar, Y. L., Caneba, G. T., Chem. Eng. Commun., 2000, 189, 571.

25. Tirumala, V. R., Caneba, G. T., Dar, Y. L., Wang, H. H., Mancini, D. C., $A d v$. Polym. Tech., 2003, 22, 126. 
26. Kabra, B. G., Gehrke, S. H., "Microporous fast response Gels and Methods of Use", U. S. Patent No. 6,030,442, 2000, February 29.

27. Kim, B. S., Chiba, T., Inoue, T., Polymer, 1995, 36, 43.

28. Kim, S., An, J. H., J. Appl. Polym. Sci., 1995, 58, 491.

29. Talroze, R. V., Kuptsov, S. A., Sycheva, T. I., Shandryuk, G. A., ACS Symp. Ser., 1996, 632, 304.

30. Abeysekera, R., Bushby, R. J., Caillet, C., Hamley, I. W., Lozman, O. R., Lu, Z., Robards, A. W., Macromolecules, 2003, 36, 1526.

31. Otmakhova, O. A., Kuptsov, S. A., Talroze, R. V., Patten, T. E., Macromolecules, 2003, 36, 3432. 


\section{Chapter 6}

\section{A SANS Study of Poly (N-Isopropylacrylamide) \\ Gels from Synchrotron-Radiation-Induced \\ Polymerization}

\section{Introduction}

Hydrogels are polymers that can absorb water many times their own volume. Depending on their inherent structure, these polymers can be tuned to respond to a variety of external stimuli. The response of hydrogels and their transition from a hydrophilic to hydrophobic state has been widely studied using scattering techniques. ${ }^{1-12}$ Phase transition varies with the morphology of the polymers, which depends on the chemical composition and synthesis conditions. For example, an increase in crosslinker concentration (crosslink density) leads to faster transition rates. Most of the hydrogels studied earlier were synthesized by chemical initiation below the lower critical solution temperature (LCST), although chemical syntheses above the LCST have recently gained interest in the scientific community due to their faster response characteristics. Efforts to develop gels with fast response led to synthesis either deep in the phase envelopes (above 
the LCST or below the upper critical solution temperature) and even mixed exposure to phase envelopes. ${ }^{13-16}$ It is now widely known that gels prepared in a hydrophobic environment possess faster swelling transitions.

Poly (N-isopropylacrylamide) (PNIPAm) is a well-studied system that can reversibly respond to a change in temperature, a so-called thermoreversibie hydrogel. PNIPAm has an LCST in water at $32^{\circ} \mathrm{C}$, above which it turns hydrophobic and thus collapses by squeezing out the previously absorbed water. Kabra and Gehrke produced gels with fast response rates by polymerizing NIPAm above its LCST at $32^{\circ} \mathrm{C}$. Temperature-induced morphological collapse of such polymers is routinely characterized by gravimetric analysis or optical microscopy, although only macroscopic size change is deduced from either technique. The problem was explored earlier using small angle scattering, but the results were not translated into insightful analysis. ${ }^{17,18}$ Unless the gels offer good contrast to the probing beam, for example, as a chemically heterogeneous phase, the small angle scattering profile of gels ranges over many orders of magnitude without any clear information on their form. A recent small angle neutron scattering study characterized the structural variation of hydrogels with solvent (water) concentration. These gels were made in the presence of ionic moieties (sodium acrylate), which offer excellent scattering contrast to the probing beam. The change in intensity with change in moisture content was therefore attributed to the growth and disappearance of hydrophobic and ionic islands within the gel network. Synthesis of hydrogels was also frequently carried out by gamma-ray irradiation process. The so called $\gamma$-gels were also studied by small angle neutron scattering to differentiate their chemical structure from that of chemicallygenerated network. ${ }^{7,11}$ Since the investigated wave vector was in the medium-Q range, 
the scattering profiles were analyzed to find the crosslink density of the mesh, as opposed to an absolute characterization of the pore-size.

The hydrogels employed in this study were synthesized by x-ray-induced polymerization above the LCST, wherein monomers were irradiated with hard x-rays from synchrotron radiation in the absence of any chemical additives or initiators. An xray-induced synthesis is quite different from a chemical synthesis in that the initiation rate is spatially uniform and constant throughout the reaction, resulting in uniformly branched high molecular weight products. We recently synthesized spatially defined hydrogel microstructures, in which spatial confinement was shown to result from the reaction mechanism. ${ }^{19}$ By combining a so-called free radical retrograde precipitation polymerization process ${ }^{20}$ with the concept of deep $\mathrm{x}$-ray lithography, polymeric microstructures were spatioselectively fabricated in situ and later incorporated into microchips for use in a variety of lab-on-chip and controlled release applications. The stimuli response of such gels is, therefore, of utmost importance, since the change in morphology depends on the synthesis strategy. Gels prepared in an inverse precipitation regime have heavy volume shrinkage and thus possess faster response rates owing to increased solvent mobility through the network. ${ }^{21}$ Swelling transition of such hydrogels can be deduced from a change of scattering profile with temperature. Above the LCST, PNIPAm collapses by excluding the solvent from its environment and increases the scattering contrast to neutrons that encounter the solvent exclusive of the gel network. On the contrary, water is absorbed within the gel network in a swollen state below the LCST, decreasing the scattering contrast. The change in intensity at any given temperature relative to that of a swollen network at room temperature thus provides both qualitative 
and quantitative information about the influence of temperature on excluded solvent or the change in pore size.

\section{Experimental}

\section{Materials Used}

N-isopropylacrylamide (NIPAm) $(>99 \%$, stabilized) was purchased from Arcos Organics Ltd. while N,N-Methylene bisacrylamide (MeBAm) and deuterated water were purchased from Aldrich Chemical Company. Unless otherwise mentioned, the water used in all procedures was deionized.

\section{Synchrotron-Radiation-Induced Polymerization}

Synthesis experiments were conducted at the 2-BM-B bending magnet beamline of the Advanced Photon Source. ${ }^{22}$ Used in a "pink" beam configuration, x-rays were configured
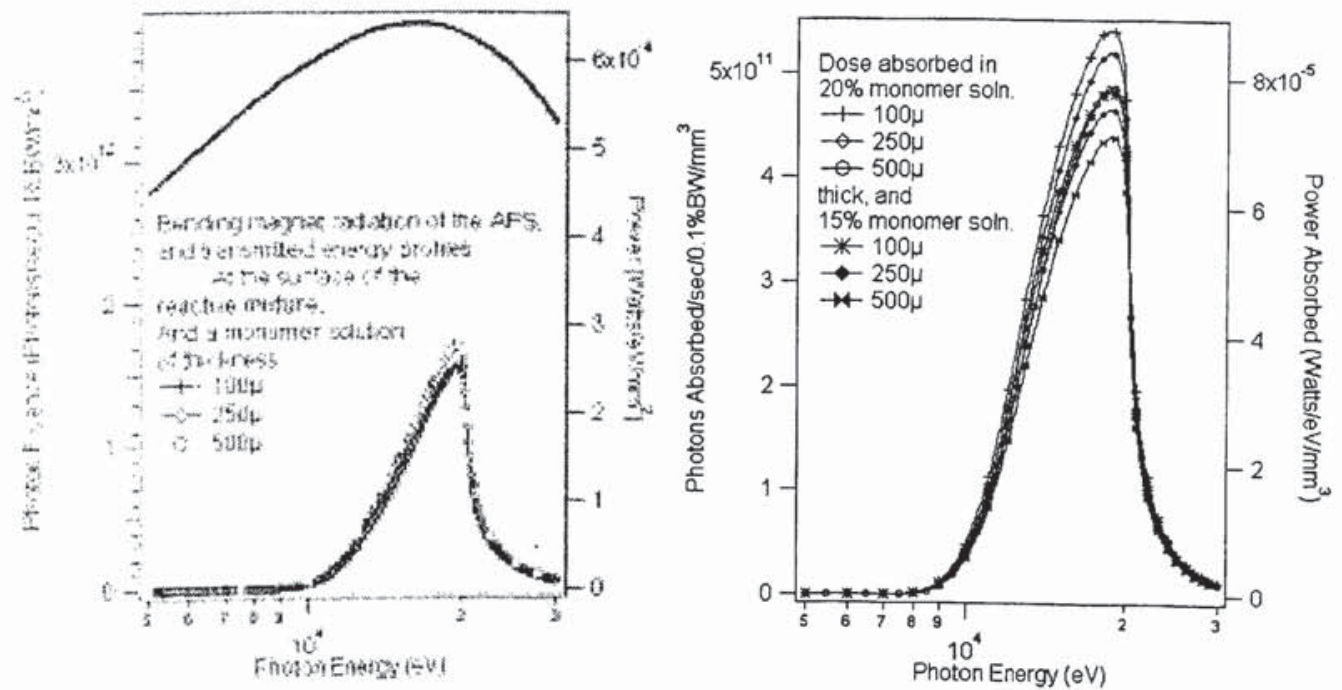

Figure 6.1: Energy spectra of the bending magnet radiation of the Advanced Photon

Source and the transmitted energy profiles through monomer solutions of various thicknesses (left) and their corresponding absorption profiles (right). 
to a narrow photon energy range of $10-20 \mathrm{keV}$, by passing them through a series of optical elements placed in the path of the beam. Radiation was first passed through one-millimeter graphite filter, which cuts off the low-energy radiation $(<5 \mathrm{keV})$. It was then reflected off a chromium mirror held at $0.15^{\circ}$ from the horizontal, to cut off the high-energy radiation $(>20 \mathrm{keV})$. Attenuation past each optical element was calculated by multiplying its transmission with the incident flux on the sample. Absorption in the samples is calculated as a difference in photonic brilliance before and after passing through an element. The power absorption spectra of the monomer solutions are as shown in Figure 6.1. The overall energy absorbed by the sample is calculated from the weight average attenuation through a given thickness of monomers and water. For the optical elements and filters used in this experiments, all the samples have a photonic absorption peak at about $15-16 \mathrm{keV}$. Table 6-1 shows the photonic absorption of the monomer mixture of any given thickness.

Two monomer solutions were prepared by dissolving $15 \%$ and $20 \%$ (by weight) NIPAm, respectively, in water. $10 \% \mathrm{MeBAm}$ (wt/wt relative to NIPAm) was added to the monomer as a crosslinker. Without further addition of any other chemical additives, the reactant mixture was encapsulated within a liquid chamber consisting of two silicon wafers. The wafer assembly was placed on an aluminum backing plate, maintained at $45^{\circ} \mathrm{C}$, and was exposed to hard x-rays whose energy profile is given in Figure 6.1. Monomers were irradiated for a predetermined reaction time, which also corresponds to the absorbed dose. Gels thus produced were stored in water until further use.

Small Angle Neutron Scattering 
Table 6-1. SANS studies performed on samples at different temperatures

\begin{tabular}{|l|l|l|l|l|l|}
\hline & 1000 a.u. & 2000a.u. & 3000a.u. & 4000a.u. & 5000a.u. \\
\hline $25^{\circ} \mathrm{C}$ & $0 / \mathrm{x}$ & $0 / \mathrm{x}$ & $0 / \mathrm{x}$ & $0 / \mathrm{x}$ & $\mathrm{x}$ \\
\hline $30^{\circ} \mathrm{C}$ & $\mathrm{o} / \mathrm{x}$ & $0 / \mathrm{x}$ & $0 / \mathrm{x}$ & $0 / \mathrm{x}$ & \\
\hline $35^{\circ} \mathrm{C}$ & $\mathrm{o} / \mathrm{x}$ & $0 /$ & $0 / \mathrm{x}$ & $0 / \mathrm{x}$ & \\
\hline $40^{\circ} \mathrm{C}$ & $\mathrm{o} / \mathrm{x}$ & $0 /$ & $0 / \mathrm{x}$ & $0 / \mathrm{x}$ & \\
\hline $45^{\circ} \mathrm{C}$ & $0 / \mathrm{x}$ & $0 / \mathrm{x}$ & $0 / \mathrm{x}$ & $0 / \mathrm{x}$ & $\mathrm{x}$ \\
\hline $50^{\circ} \mathrm{C}$ & 0 & 0 & 0 & 0 & \\
\hline
\end{tabular}

$0-15 \%$ NIPAm in water; $x-20 \%$ NIPAm in water.

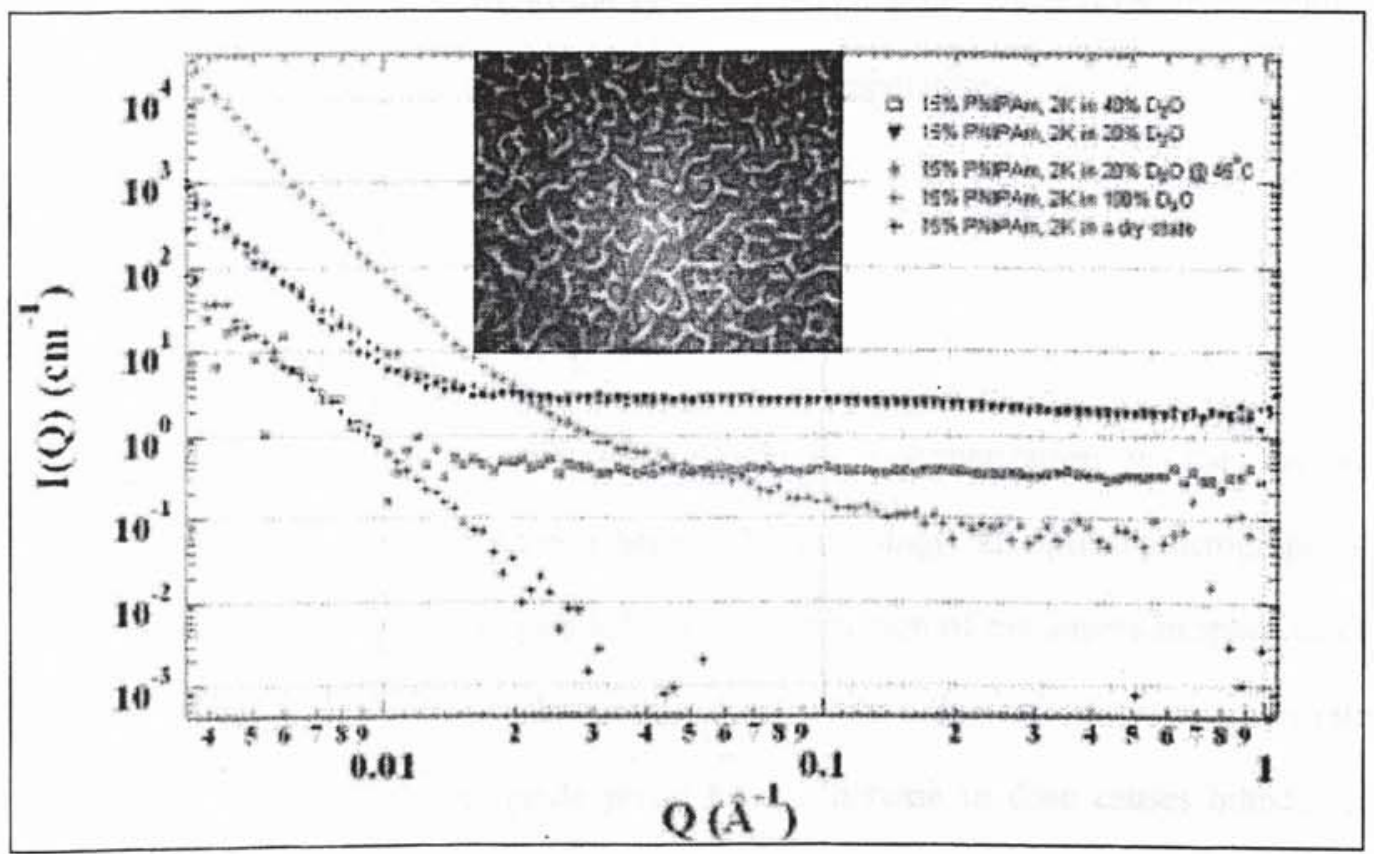

Figure 6.2: Plot of intensity versus scattering vector of samples in a swollen and dry state. Also shown in the Figure are scattering profiles from samples in mixed solvents (concentrations given in legend). Inset is an optical micrograph of a PNIPAm gel surface, prepared by x-ray irradiation @ $45^{\circ} \mathrm{C}$. 
Small angle scattering experiments were performed at the small angle neutron diffraction (SAND) instrument of the Intense Pulsed Neutron Source. ${ }^{14}$ Prior to the scattering experiments, all the gels were thoroughly dried and reswollen to equilibrium solvation in deuterated water. They were then cut into discs and transferred into 1-mm-deep quartz wells filled with $\mathrm{D}_{2} \mathrm{O}$. The sample holder was connected to a circulating water bath to help maintain the sample temperature. Samples were also equilibrated at each desired temperature before data acquisition. Small angle scattering measurements of gels with two monomer concentrations were obtained at various temperatures as shown in Table 6-1. Phase transition of gels was observed via small angle scattering by first heating the samples from room temperature to the synthesis temperature, while reversible swelling was measured by cooling the samples again to room temperature.

\section{Results and Discussion}

Initial Morphology:

Gels formed from synchrotron-radiation-induced polymerization in the inverse precipitation regime typically have a branched morphology, an optical micrograph of which is given in the inset of Figure 6.2. Uniform initiation of monomers in space leads to increased rates of termination by combination, while a decrease in propagation rate will be evidenced due to retrograde precipitation. Increase in dose causes branching, since contmuous irradiation contributes to subsequent termination between the radicals of growing chains throughout the network. The gels, therefore, have a rich morphology with pore sizes ranging over orders of magnitude from a few tens of nanometers to a few microns. The change in scattering intensity with scattering vector for samples from 15 wt $\%$ NIPAm and 2000 a.u. dose, in an equilibrated state and a dry state, at $30^{\circ} \mathrm{C}$ is shown 
in Figure 6.2. Scattering from samples solvated in $\mathrm{D}_{2} \mathrm{O}$ was also contrasted with that of samples solvated in a mixed solvent of deuterated and hydrated water of known proportions (Figure 6.2). Gels in a dry state scatter with a high power-law slope of $\sim 4$, which is the result of the porous gel morphology appearing as a complex fractal of higher dimensions. The smallest channel available for fluid flow is approximately $12-15 \mathrm{~nm}$, which corresponds to a breakdown in the dry gel scattering at $\mathrm{Q} \sim 0.006 \AA^{-1}$.

\section{Data Reduction:}

Small angle scattering data from samples are treated by subtracting the background scattering from the instrument and the solvent. Ideally, this should provide structural information of the gels in the range of $Q$ being investigated. The presence of deuterated water in the network causes excess scattering from the samples and results in unusually high power-law slopes compared to those from a dry gel. These gels are also characteristic of pore sizes ranging over an order of magnitude, which will be seen by the probing beam as fractals with higher dimensions. Consequently, an absolute characterization of sample morphology from the scattering curves is not possible. Nevertheless, the impact of phase separation on scattering from the samples can still be ascertained after some data manipulation. The change in scattering of gels at any given temperature was emphasized by normalizing the intensity of scattering with respect to the scattering from the same sample at room temperature. Mathematically, this can be calculated as:

$$
I(Q)_{\text {relative }}=\frac{I(Q)_{T}-I(Q)_{R T}}{I(Q)_{R T}}
$$


Small angle scattering profiles of gels can now be analyzed for a change in scattering intensity induced by change in morphology, which increases contrast in scattering from the sample.

\section{Change in Morphology}

The influence of temperature, which brings about a change in gel morphology by excluding the absorbed solvent, can be seen as a change of contrast and thus scattering intensity. Since an increase in temperature induces an increase in scattering contrast due
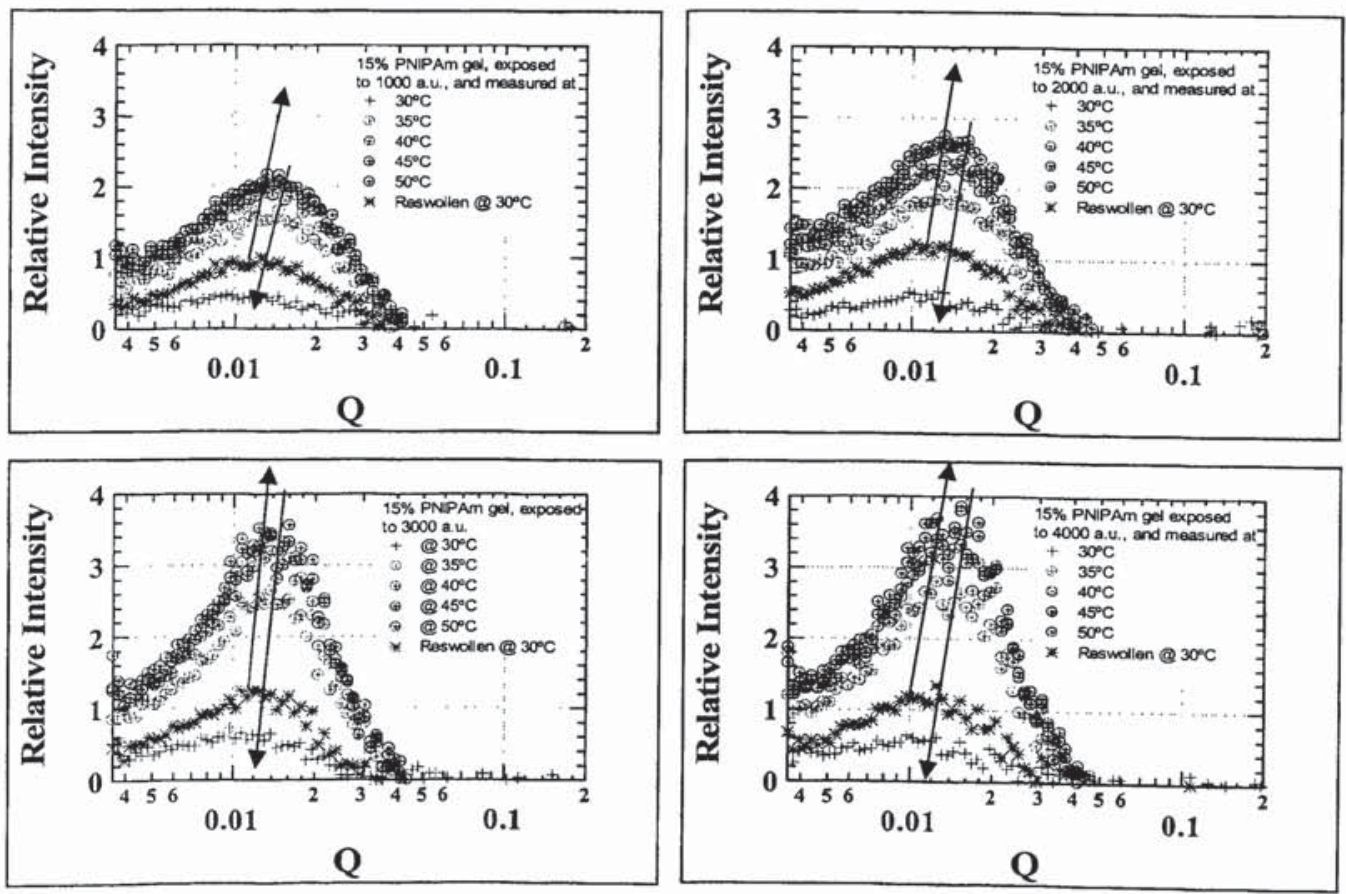

Figure 6.3: Relative intensity plotted against scattering vector at various temperatures

$30,35,40,45,50^{\circ} \mathrm{C}$ and reswollen at $30^{\circ} \mathrm{C}$. Plots are for samples synthesized from $15 \%$ NIPÀm at an overall exposure dose of 1000 a.u. (top, left), 2000 a.u. (top, right), 3000 a.u. (bottom, left) and 4000 a.u. (bottom, right). Up arrow represents the change in scattering profiles during the heating cycle from $25^{\circ} \mathrm{C}$ to $50^{\circ} \mathrm{C}$, while down arrow represents the cooling cycle from $50^{\circ} \mathrm{C}$ to $30^{\circ}$. 


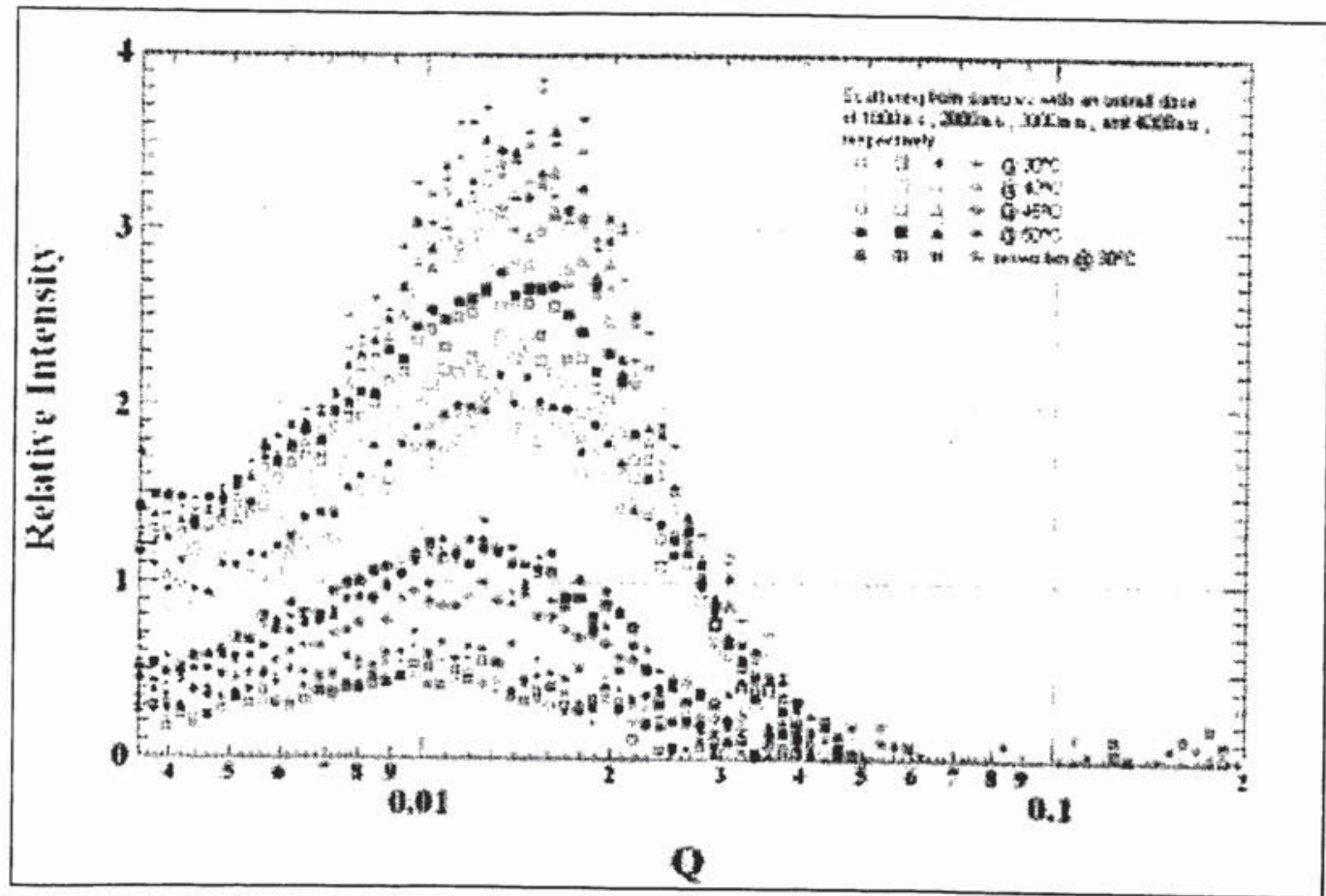

Figure 6.4: Scattering profiles re-plotted for gels made from $15 \%$ monomer concentration, at various doses and temperatures.

to phase separation, the relative intensity of gels should increase in the range of $\mathrm{Q}$ within which the chain collapse is significant. It is therefore interesting to find that, at temperatures above the LCST of the PNIPAm-water system, a peak in relative intensity emerges at $Q \sim 0.015 \AA^{-1}$ (Figure 6.3). The broadness of this peak also defines the characteristic size of the solvent excluded from the network. It can be noted from Figure 6.3 that the gels irradiated to a higher dose have a relatively narrow peak in intensity, which can be interpreted as corresponding to the size distribution of the deswollen domains. Gels irradiated to higher doses will have relatively smaller pores with a narrow pore size distribution as a result of increased branching. The solvent excluded from such a network will thus be of the same size as that of the pores. The effect of dose on swelling transition can be observed by plotting scattering profiles 
of gels prepared at different doses on the same plot (Figure 6.4). Below the LCST, which for PNIPAm-water is about $32-34^{\circ} \mathrm{C}$ in water, gels irrespective of irradiated dose have the same swollen morphology. On the contrary, deswelling above the LCST increases with an increase in overall dose, although gels with a dose greater than 3000 a.u. seem to collapse to same extent. The extent of macroscopic size change with temperature can also be estimated from the value of relative intensity at very low $Q$. Since the gels are prepared in an inverse precipitation regime, their macroscopic morphology will be the same at all temperatures above the LCST, which can also be deduced from the same intensity at elevated temperatures. On the other hand, when initially formed, gels irradiated to higher doses are characteristic of increased branching and hence smaller effective pore size, onto which they collapse above the LCST. The role of dose in radiation-induced gel synthesis is thus similar to that of crosslinker concentration in conventional chemical synthesis of gels. An increase in dose increases branching (crosslink density) even for the same crosslinker concentration. Uniform dose rate (rate of initiation) throughout the reaction also means that, for the same crosslinker concentration, gels from radiation-synthesis will be of higher crosslink density (branching) than those from chemical syntheses, which usually are characteristic of depleting initiator efficiency. Similarly, an increase in monomer concentration, which specifies the monomer distribution in space, decreases the average pore size and thus solvent mobility in phase-separated gels.

\section{Influence of Monomer Concentration:}

To affirm the effect of monomer concentration on the gel morphology, gels made at $20 \%$ NIPAm and at different doses, were also subjected to similar small angle scattering measurements as those from the $15 \%$ monomer concentration. As is the case with gels 
made from low monomer concentration, here too, relative intensity increased with an increase in temperature above the LCST. A similar saturation in relative intensity was also observed for gels with a dose greater than 3000a.u. prepared from $20 \%$ monomer concentration. Contrarily, they show a decrease in relative intensity with an increase in dose. As seen from Figure 6.5, gels irradiated with a dose of 1000 a.u. show higher deswelling than those irradiated with higher doses, although they swell back to their initial morphology when cooled to $25^{\circ} \mathrm{C}$ (as observed from the lack of relative scattering). The macroscopic size at increased temperatures above the LCST was also not much different from that at a lower temperature as evidenced from the low initial relative intensity values. The decrease in relative intensity with an increase in dose also suggests a critical pore size, below which solvent mobility within the gel network is severely impaired. Thus, compared to the excellent swelling characteristics exhibited by the gels made at $15 \%$ monomer concentration, concentrated gels possess very low macroscopic swelling and shrinking. Monomer dissolved in the solvent at a solubility threshold, when irradiated, produces gels that are closely interconnected. Thus, pore-size distribution in concentrated gels will therefore be narrower than the gels made from dilute monomer solutions. Longer times of exposure will further increase branching and interconnectivity, thus decreasing the critical pore size of the network. However, in the case of concentrated gels, branching decreases solvent mobility affecting the phase transitions. This can also be determined from the scattering profiles of gels made at higher concentration, which above the LCST, possess lower macroscopic deswelling, shrinking to a relatively uniform final morphology (low relative intensity and narrow peaks). Figure 6.5 also shows a change in scattering peak brought about by a change in concentration. As expected, this 
relates to a smaller characteristic pore size of the network for gels with higher monomer concentration.

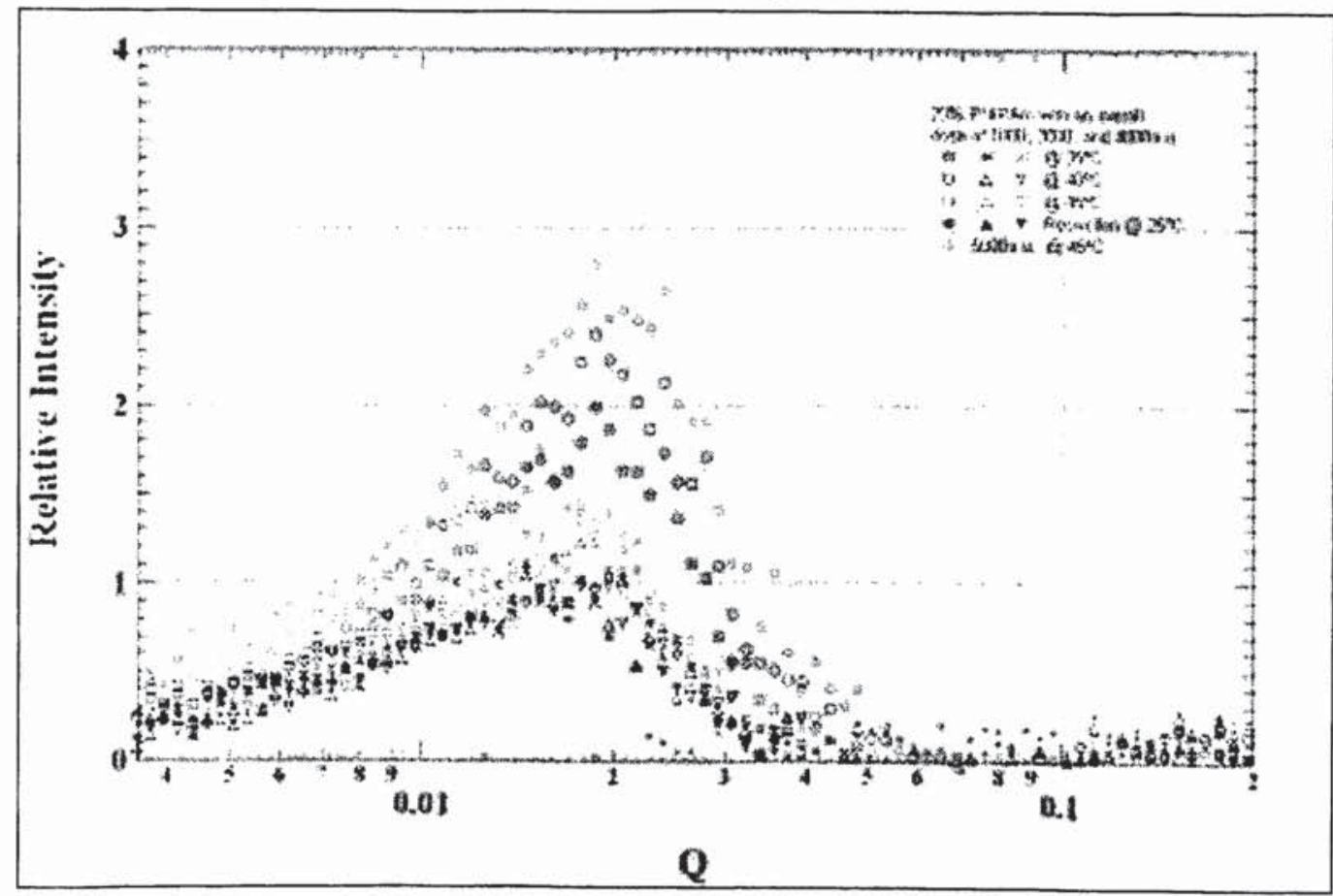

Figure 6.5: Relative Intensity versus scattering vector of gels made at $20 \%$ monomer concentration and with a dose of $1000,3000,4000$, and 5000 a.u., at various temperatures.

\section{Conclusions}

Thermoreversible hydrogels based on Poly ( $\mathrm{N}$-isopropylacrylamide) were synthesized by exposing monomers to the bending magnet radiation from a synchrotron. Polymerization in a quiescent system was carried out in an inverse precipitation regime, in which reaction propagation and termination are thermodynamically controlled. Gels thus precipitated are of a unique morphology as a result of the synthesis route. The morphological response of gels was measured by small angle neutron scattering, in which 
monomer concentration and absorbed dose (time of reaction) were found to affect the temperature-induced reversible collapse. Above the LCST, deswelling of gels made from dilute monomer solutions increased with an increase in time of exposure. However, gels made at the solubility limit of the monomer in solvent had a decreasing deswelling with an increase in dose as a result of excessive branching. As expected, pore size of dilute gels was lower than that of concentrated gels, which can be related to the spatial distribution of the monomers in solvent. Although an absolute characterization of the pore size of the networks was not made, the analysis presented here is believed to be sufficient to gain a qualitative understanding of the morphology of the hydrophobic hydrogels prepared by the synchrotron radiation induced polymerization. Average mesh size or the pore size distribution of these gels can be absolutely characterized by measuring them in the ultra-small angle region of neutron scattering $(1 \mathrm{e}-5<Q<1 \mathrm{e}-3)$, at high neutron flux instruments like the ones at National Institute of Standards and Technology or at the neutron reactor of the Oakridge National Laboratories. 


\section{References}

1. Ikkai, F.; Shibayama, M.; Han, C. C. Macromolecules 1998, 31, 3275.

2. Mikosch, W.; Geissler, E. Berichte der Bunsengesellschaft/Physical Chemistry Chemical Physics 1998, 102, 1589.

3. Koizumi, S.; Annaka, M.; Borbely, S.; Schwahn, D. Physica B: Condensed Matter, 2000, 367.

4. Kratz, K.; Hellweg, T.; Eimer, W. Polymer, 2001, 42, 6631.

5. Fernandez-Barbero, A.; Fernandez-Nieves, A.; Grillo, I.; Lopez-Cabarcos, E., Phy. Rev. E., 2002, 66, 051803.

6. Koizumi, S.; Monkenbusch, M.; Richter, D.; Schwahn, D.; Farago, B.; Annaka, M., App. Phy. A, 2002, 74, 399-401.

7. Norisuye, T.; Masui, N.; Kida, Y.; Ikuta, D.; Kokufuta, E.; Ito, S.; Panyukov, S.; Shibayama, M., Polymer, 2002, 43, 5289.

8. Shimizu, S.; Kurita, K.; Furusaka, M., App. Phy. A., 2002, 74, 389.

9. Sugiyama, M.; Annaka, M.; Maeda, Y.; Hara, K., App. Phy. A., 2002, 74, 421.

10. Sugiyama, M.; Annaka, M.; Motokawa, R.; Kuwajima, S.; Hara, K. Physica B $\checkmark$ , 2002, 311, 90 .

11. Takata, S.-I.; Norisuye, T.; Shibayama, M. Macromolecules 2002, 35, 4779. 
12. Sugiyama, M.; Annaka, M.; Hara, K.; Vigild, M. E.; Wignall, G. D. J. Phy. Chem. B, 2003, 107, 6300 .

13. Kabra, B. G., and Gehrke, S. H. Polym. Comuni. 1991, 32, 322.

14. Kabra, B. G., and Gehrke, S. H.: USA, United States Patent No. 6,030,442, February 29, 2000.

15. Hirasa, O., Ito, S., Fujishige, S., and Ichijo, H. Polymer Gels; Plenum Publishers: New York, NY, 1991.

16. Wu, X., Hoffman, A. S., and Yager, P. J. Polym. Sci.: Part A, 1992, 30, 2121.

17. Thiyagarajan, P., Personal Communication, 1998.

18. Ilavsky, J., Personal Communication, 1999.

19. Tirumala, V. R., Caneba, G. T., Dar, Y. L., Wang, H. H., and Mancini, D. C. Adv. Polym. Tech., 2003, 22, 126.

20. Caneba, G. T. Adv. Polym. Tech 1992, 11, 277.

21. Walther, D. H., Blanch, H. W., and Prausnitz, J. M.; Lawrence Berkeley Laboratory: Berkeley, 1993.

22. Lai, B., Mancini, D. C., Yun, W., and Gluskin, E., Proc. SPIE., 1996, 2880, 171. 


\section{Chapter 7}

\section{Lithographically-Assisted Synthesis of High}

\section{Aspect Ratio Hydrogel Microstructures}

\section{Introduction}

Environmentally sensitive hydrogels are polymers that respond to external stimuli with a change in their internal morphology. External stimuli could be any or combination of properties like temperature, $\mathrm{pH}$, concentration of the additives, light intensity, etc. ${ }^{1-5}$ Since the change in enthalpy of these hydrogels due to an external stimulus is usually very high, they are highly applicable as sensors, controlled drug-delivery systems or actuators. $^{6}$ Conventional hydrogel synthesis usually results in bulk gels with no spatial control (unless synthesized in molds), and thus such gels cannot be used in micro- or nanoscale devices unless they are made by photopolymerization techniques. ${ }^{7,8}$ Nevertheless, they are usually of low aspect ratio due to the limitations arising from photolithography.

Deep x-ray lithography (DXRL) is conventionally used to make metal microstructures, to be embedded in microelectromechanical systems. ${ }^{9}$ In DXRL, photoresists are usually 
exposed into desired patterns by spatially masking out the incident $\mathrm{x}$-rays. By adopting the masking technique from deep x-ray lithography, a unique reaction pathway based on synchrotron-radiation-induced polymerization chemistry was proposed, in which a freeradical reaction pathway was employed. ${ }^{10}$ We have also recently shown that the reaction propagation in free-radical polymerization can be inherently controlled by thermodynamically controlling the extent of reaction spread. ${ }^{11}$ By bringing these principles together, we ventured into study of the spatially-defined polymerization of hydrogels based on $\mathrm{N}$-isopropylacrylamide (NIPAm) and methacrylic acid (MAA).

\section{Exposure Station Configuration}

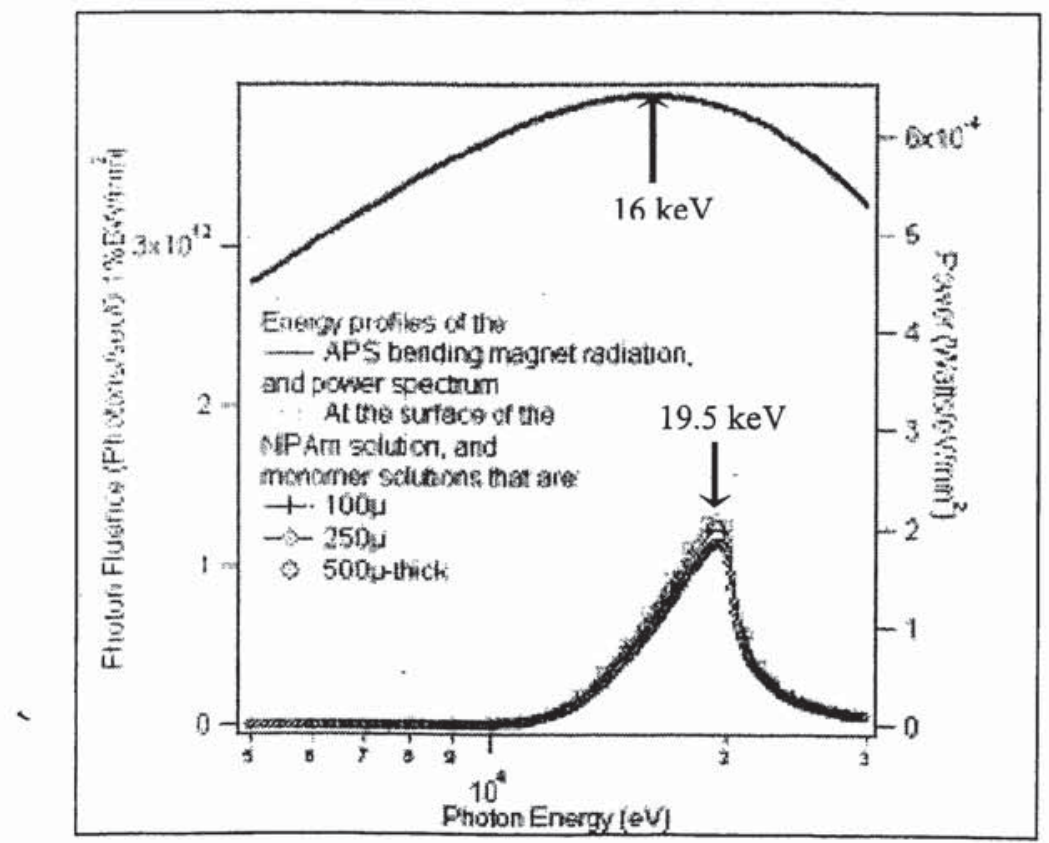

Figure 7.1: Power spectrum of the bending magnet radiation at the APS, and the incident and transmitted energy profiles through monomer solutions of various thicknesses. 
All exposures were carried out at the deep x-ray lithography exposure station of the bending magnet beamline, 2-BM-B of the Advanced Photon Source. ${ }^{12}$ The photon energy range of the incident radiation was tuned to wavelengths of choice by having a one-

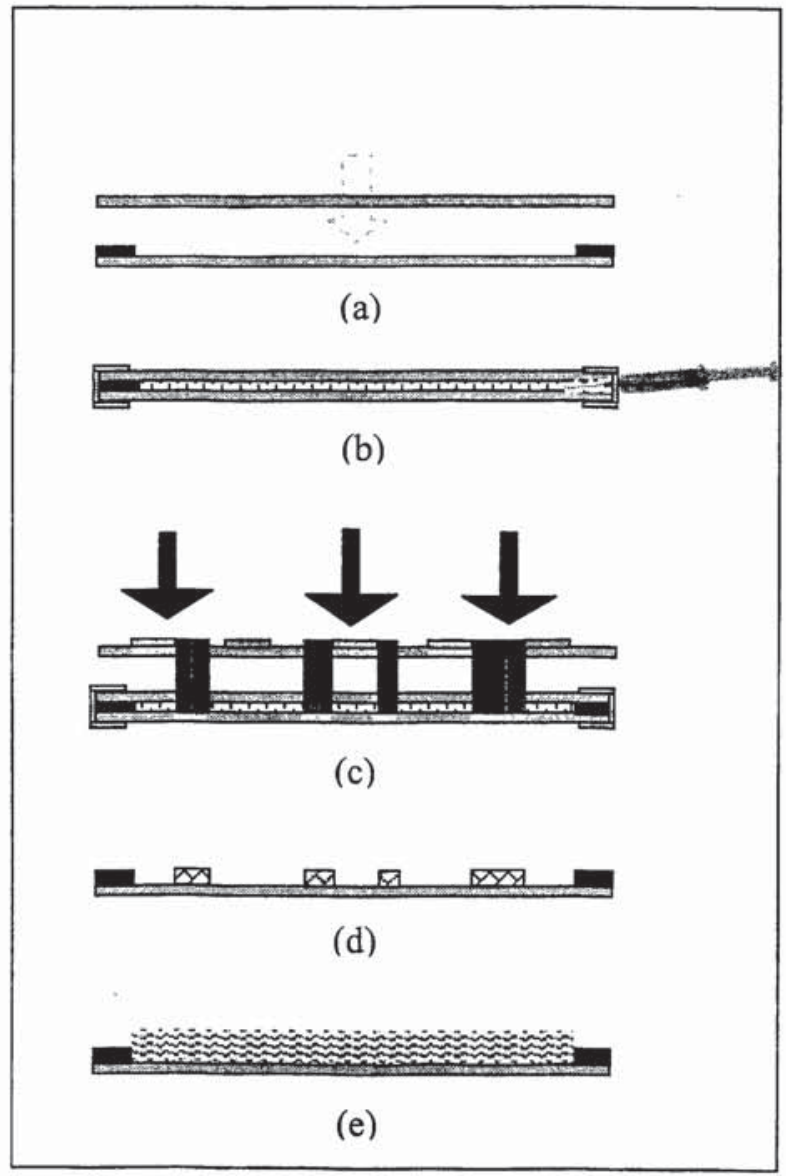

Figure 7.2: Schematic of the process methodology showing (a) the liquid containment chamber, made up of two silicon wafers and a spacer, (b) monomer solution injected into the wafer assembly, (c) polymerization initiated by x-ray exposure through a mask, (d) polymer patterns obtained by drying out the solvent, and (e) swollen patterns when exposed to a solvent. Note that the swelling is also thermoreversible.

millimeter-thick carbon filter, a chromium mirror held at $0.15^{\circ}$ from the horizontal, and two beryllium windows $250 \mu$ and $125 \mu$ thick (used to cap the beamline at both ends), in 
the path of the beam. Figure 7.1 depicts the bending magnet radiation profile of the Advanced Photon Source and the transmitted energy profiles as the beam passes through the optical elements mentioned above. While all the experiments were performed using the setup described, the energy profile of incident $\mathrm{x}$-rays can be modified by changing either the aperture or the optical elements as desired. A change in the overall dose of exposure does not affect the reaction chemistry, although it defines the spatial resolution and the product morphology. It was therefore deemed necessary to study the process at an optimized beamline configuration, which resulted in a narrow bandwidth for energy absorption. The experimental setup on the beamline was similar to that of the deep x-ray lithography exposures ${ }^{9}$ except for the sample holder.

Reactive mixtures were prepared by dissolving known amount of monomers in water. Stimuli-response of gels largely depends on the physical and chemical structure of the gel

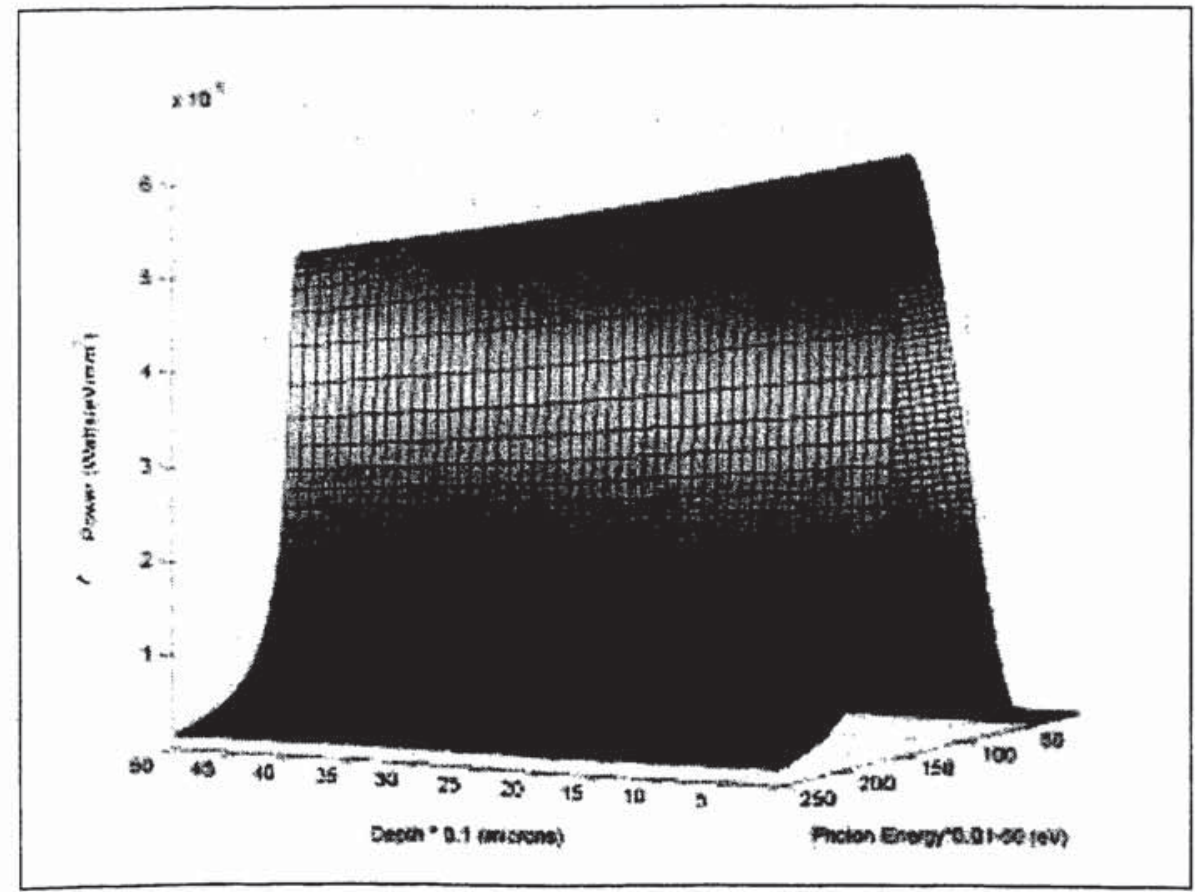

Figure 7.3: Dose absorption profile in a 500 $\mu$-thick monomer solution of NIPAm. 
network, which depends on the initial monomer concentration. Monomer solutions then were captured between two silicon wafers separated by a kapton ${ }^{\otimes}$ spacer, and the edges were then sealed with Teflon ${ }^{\circledR}$ thread seal tape and a kapton ${ }^{\circledR}$ tape (Figure 7.2). The thickness of the spacer determines the height of the patterns and thus their aspect ratio. Use of a narrow vertical slit $(2 \mathrm{~mm})$ results in an incident energy profile with a peak at $16 \mathrm{keV}$, while the use of silicon wafers as a part of the sample holder ensures that the incident radiation is of narrow photon energy range (10-20 keV) (Figure 7.1). Energy deposition from such a focused high energy spectrum results in chemical events with minimum undesired or side reactions. Depending on the monomer properties, the formed polymer might further crosslink or scission beyond a critical absorbed energy.

Spatioselective reaction initiation was realized by employing an $\mathrm{x}$-ray mask during irradiation, wherein the reaction propagation will be spatially controlled by the interplay

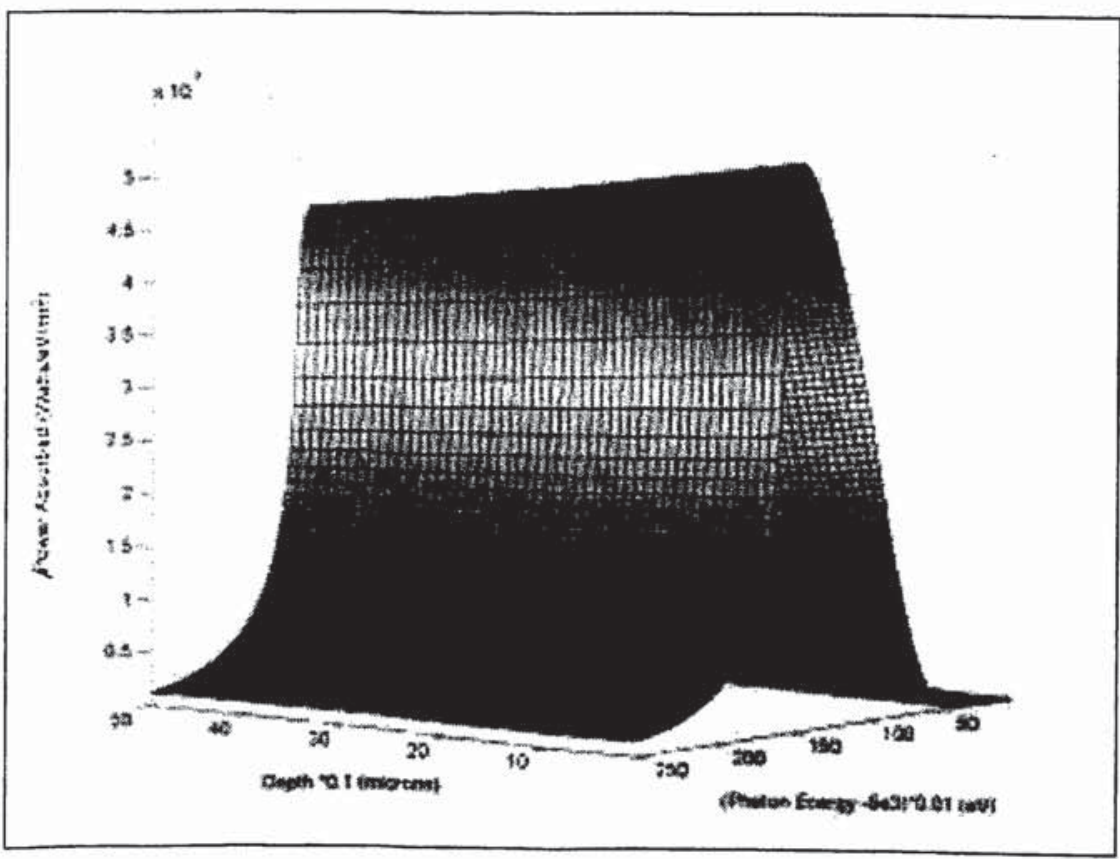

Figure 7.4: Dose absorption profile in $20 \%$ solution of MAA in water. 
between phase equilibrium thermodynamics and the exothermic chain reaction of the polymer-solvent system. Water being the predominant component of reactive mixture alloves us to produce very high aspect ratio hydrogel microstructures because of its relatively low absorption coefficient $(\sim 20 \%)$, even in $500 \mu$ thick patterns (Figure 7.3 , 7.4). Note also that the absorbed energy is quite uniform, thus producing gel microstructures of very high aspect ratio with uniform response rates to external stimuli (due to similar physical structure through their depth).

\section{High Aspect Ratio Hydrogel Microstructures}
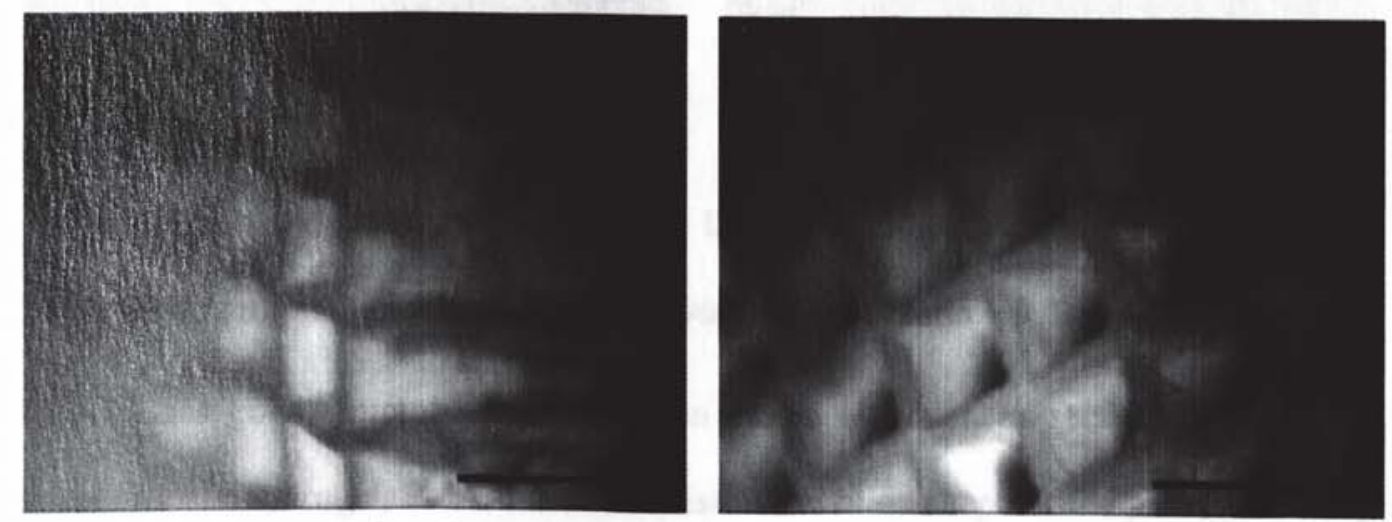

Figure 7.5: Optical micrographs of $40 \mu$-wide, $500 \mu$-thick line patterns of Poly (NIPAm). Image to the left is a depth perspective of the image to the right. Bar is $400 \mu$.

Hydrogel microstructures were created by hard x-ray-induced polymerization of monomers in water. A gold mask comprising $40 \mu$-square blocks with a $500 \mu$ pitch was used for exposing monomer solutions of varying thicknesses. Polymerized patterns were then released by dismantling the wafer assembly and were air-dried in a fume hood. Poly (NIPAm), being a thermoreversible hydrogel, induces postfacto structural imperfections 
during drying. However, when reswollen in water, the patterns regain their original shape, albeit of different size. Pattern replication aspects of NIPAm polymerization cannot be determined due to its thermoreversibility in both hydrophilic and hydrophobic solvents. Nevertheless, dried patterns were found to replicate the features well enough to validate the reaction scheme (Figure 7.5). Unlike poly (NIPAm), which imparts postfacto structural distortions on account of its thermoreversibility, poly (methacrylic acid)
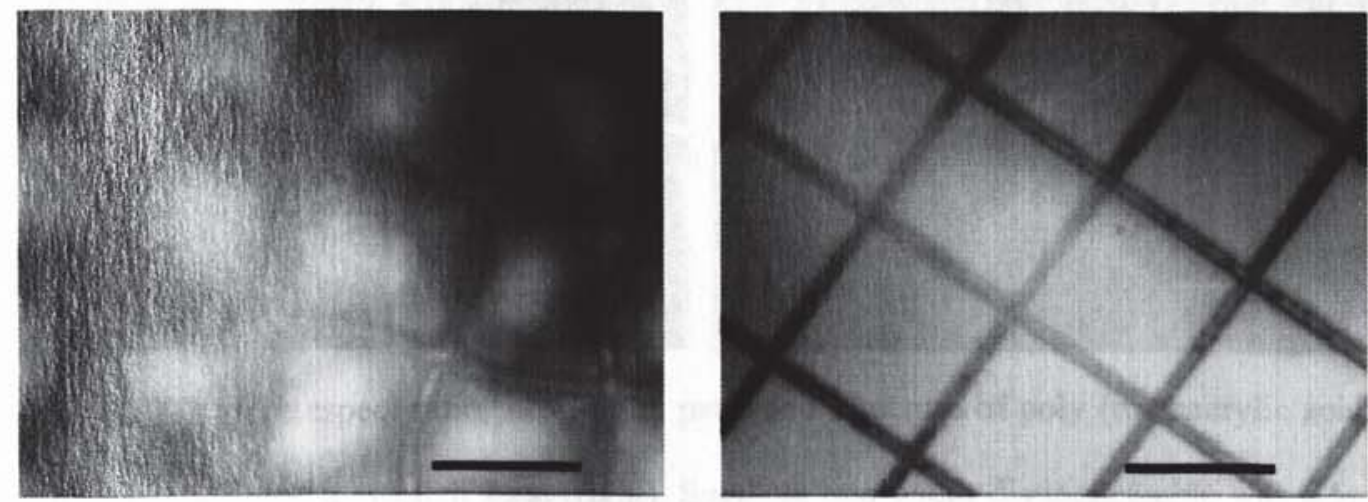

Figure 7.6: A line pattern with $30 \mu$-wide lines and $500 \mu$ square pitch replicated in Poly (NIPAm) (left) and PMAA (right), with an aspect ratio $\sim 9$. Bar $=500 \mu$.

(PMAA) has good structural stability when stored in water, as seen from figure 7.6. Being an ionic hydrogel, PMAA does not distort the basic shape of the patterns although it swells into a more relaxed state with larger feature sizes when exposed to a hydrophilic environment (Figure 7.7).

Of the various parameters affecting the process, ionization of monomers was found to be playing a crucial part in defining pattern transfer accuracy. Polymer precipitation is not effective in minimizing ionization as it is with radical trapping. Therefore, methacrylic acid patterns were initially found to be encompassed by a transparent film polymerized by chain transfer to solvent due to ionization, decreasing the contrast in resulting patterns. Without going into the details of reaction chemistry here, it suffices to 
say that the reaction spread can be minimized by irradiation at elevated temperatures. ${ }^{13}$ Thus, in principle, polymerization of MAA can also be controlled to produce spatiallydefined, well-contrasted microstructures. We are currently investigating the effect of temperature and polymerization sensitivity of this novel lithographically-assisted patterned polymer synthesis.
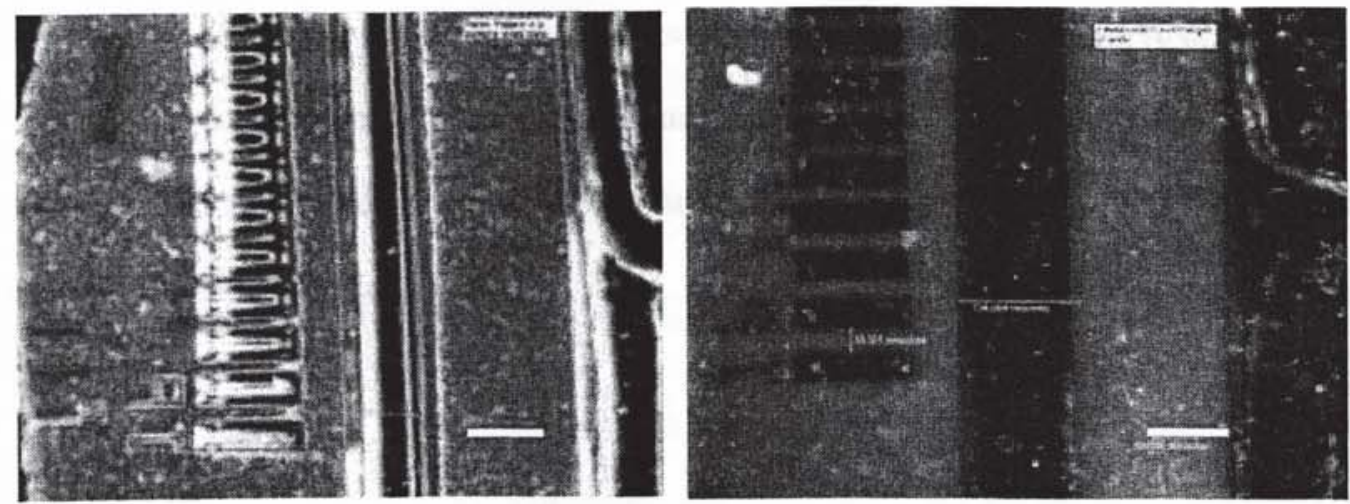

Figure 7.7: High aspect ratio, $125 \mu$-thick patterned structure of poly (methacrylic acid) in a dry (left) and a swollen state (right). Smallest line-width distinguishable was $6.5 \mu$. The $16 \mu$-wide line in a dry state swells up to $30 \mu$ when swollen. $\mathrm{Bar}=100 \mu$.

\section{Discussion}

We have shown for the first time that chemical synthesis can be combined with lithographic approach to yield novel "smart" materials, with special characteristics. Using hydrogels as an example, we have demonstrated that by choosing the right chemistry, the potential of lithography can be greatly exploited in the generation of novel microstructures based on soft materials. Microgels, due to their small size, respond much faster to an external stimulus compared to bulk gels. Gel microstructures, or to be precise, microgel structures, therefore, can find a niche as micro- and nanosensors, controlled release carriers and targeted drug delivery systems. The polymer backbone 
making up the gel structure can be carefully tailored to enable unique functionalities. For example, grafting the microgel structures with a biocompatible, poly (ethylene glycol) enables them to target specific biological components for coupling. Copolymerizing a thermoreversible component and an ionic component, microgel structures can be tailored to respond to both a change in temperature and $\mathrm{pH}$. Polymerizing a monomer in the presence of a metal nanoparticle solution, for example, results in microgel structures that can respond to an external electronic or magnetic trigger. We are currently working towards developing unique "smart" micro- and nanogel structures that are biocompatible and are responsive to multiple stimuli.

\section{Conclusions}

A new approach towards synthesis of high aspect ratio polymeric microstructures was described. Hydrogels, on which spatial conformation is hard to impose, were polymerized using this method to form spatially well-defined microstructures. Polymer patterns of high aspect ratio were synthesized by using water as a polymerizing medium. Water, with its low absorption coefficient, provides relatively uniform energy distribution, and thus uniform reaction profile, through its depth. High absorption crosssection of water thus allows extending this concept to the synthesis of very high aspect ratio structures. Although polymer patterns were prepared in a collapsed state, poly (NIPAm) swells back in water due to its thermoreversibility while PMAA preserves its structure, unless dehydrated. Future work involves improving image contrast in PMAA and studying the effect of process parameters on pattern transfer and gel response to external stimuli. 


\section{References}

1. Yu. H., and Grainger, D. W., J. Appl. Polym. Sci., 1993, 49 (9), 1553.

2. Hirokawa, Y. and Tanaka, T., J. Chem. Phys., 1984, 81, 6379.

3. Tanaka, T., Phy. Rev, Lett., 1980, 45, 1636.

4. Miyata, T., Asami, N., and Uragami, T., Nature, 1999, 399, 766.

5. Qiu, Y., and Park, K., Adv. Drug Delivery Reviews, 2001, 53, 321.

6. Hoffman, A. S., Clinical Chem., 2000, 46, 1478.

7. Beebe, D. J., Moore, J. S., Bauer, J. M., Qing, Y., Liu, R. H., Devadoss, C., Byung-Ho, J., Nature, 2000, 404, 588.

8. Liu, V. A., and Bhatia, S. N., Biomedical Microdevices, 4:4, Kluwer Academic Publishers, Netherlands, 2002, 257.

9. Mancini, D. C., Moldovan, N. A., Divan, R., De Carlo, F., and Yaeger, J., Proc. SPIE, 2001, 4557, 77.

10. Tirumala, V. R., Caneba, G. T., Dar, Y. L., Mancini, D. C., and Wang, H. H., Adv. Polym. Tech., 2003, 22, 126.

11. Çhapter 4, This Dissertation

12. Lai, B., Mancini, D. C., Yun, W., and Gluskin, E., Proc. SPIE, 1996, 2880, 171.

13. Moore, P. W., J. Polym. Sci., Polym. Chem. Ed., 1983, $21,2491$. 


\section{Chapter 8}

\section{Conclusions and Future Directions}

Free-radical kinetic events, which are notorious for their "run-away" behavior, can be inherently controlled without the use of additives or any other chemical agents, by choosing a proper solvent and temperature. The otherwise uncontrolled and exothermic chain propagation can be controlled to limit itself to a few tens of nanometers, by polymerizing the monomer in a quiescent environment of a solvent with inverse temperature solubility behavior. Among the class of the so-called controlled polymerization chemistries, the effect of mixing in controlling the chain growth is a feature unique only to the FRRPP process. Polymer chain growth in quiescent FRRPP systems was also experimentally determined to be diffusion-controlled from the timeresolved small-angle $\mathrm{x}$-ray scattering techniques. The reaction pathway of FRRPP systems remains the same irrespective of the method of initiation, extending the aspects of controlled polymerization to the radiation-induced polymer synthesis. Combining the reaction chemistry prescribed by FRRPP with the principles of deep x-ray lithography 
(spatioselective initiation with high-energy radiation), the polymerization reaction can now be self-limited only to the spatially predefined irradiated zones. This enables one to synthesize, not microfabricate, polymeric microstructures. Applying this mechanistic understanding to the synthesis of hydrogels results in spatially-controlled synthesis of microgel structures, which is possible only when the gelation is carried out in molds.

Thus, the reaction chemistry of FRRPP, unique with its thermodynamically-controlled propagation and termination reactions, was studied and was extended to a novel radiation induced polymer synthesis. The technique established in this study can now be studied in depth and applied to the synthesis of novel nanostructured materials.

\section{Future Directions}

\section{Folding Kinetics of a Polymerizing Chain in a Poor Solvent}

Even after a decade of exploration into reactive precipitation in a poor solvent, it is clear that there is little insight into the dynamics of a growing polymer chain in a poor solvent. Many researchers were successful in obtaining chain-collapse information above the LCST, but the kinetics investigated were either at a particular concentration, molecular weight, or temperature. With the advent of state-of-the-art scattering facilities, it is now feasible to resolve the problem using time-resolved small-angle scattering of a reactive system, one of which was also a part of the current study. A growing chain in a poor solvent will have the exothermic chain reaction (increasing the chain length) and the increase in molecular weight (globular collapse by excluding the solvent) acting against each other. It is essential to do a combinatorial small-angle scattering study, to completely delineate the contribution of reaction and precipitation terms to the increase 


\section{Conclusions}

or decrease of observed chain length. Even though the observed chain length will be the upper limit of a truly precipitated growing chain in a poor solvent, the effect of kinetics and thermodynamics in both the cases remains the same.

Once the effects of reaction and precipitation on chain growth are decoupled, a novel kinetic model can be developed, which takes the effect of precipitation into account after each kinetic event. This kinetic model can then be integrated into the simulation procedure presented in this study, to model and simulate the FRRPP kinetics along with growing chain behavior and its impact on product properties.

\section{Synthesis of Nanocomposite Nanoparticles}

Based on the conceptual understanding of this study, polymeric globules can also be synthesized in the presence of a known amount of inorganic nanoparticles. Nanoparticles can be based on any material that does not have an adverse affect on the reaction chemistry. For example, by polymerizing a monomer in the presence of inorganic clay, organic-inorganic nanocomposites can be produced. Similarly, one can synthesize nanohybrid particles by co-precipitating metal nanoparticles with the polymer. Since the organic and inorganic components are covalently bound in the synthesis phase, these nanocomposite materials should possess superior properties when compared with those made by conventional procedures like blending. The only competing approach to this process would be the one that involves ultrasonic methods. 
Conclusions

\section{Acknowledgments}

This project was partially supported by the National Science Foundation and the U.S.

Department of Energy. Use of the Advanced Photon Source was supported by the Department of Energy, Office of Science, Basic Energy Sciences, under contract number W-31-109-ENG-38. 


\section{Appendix I}

\section{MATLAB Code for FRP Simulations}

Main program:

$\%$ Declaration of Variables

global N T Cmon0 epsl Beta parmA parmB R kp0 kt0 kd thetat thetap F Cinit0 A1

$\mathrm{N}=10 ; \quad \mathrm{T}=323.16 ; \quad \operatorname{dens} \mathrm{P}=1.2$;

densM=0.973-1.164e-3*(T-273.16);MWmon=100.13;

Cmon0=densM*1000/MWmon; $\quad$ epsl=(densP-densM)/densP;

vols $=0$;

$\operatorname{vol} 0=0.132$

$\mathrm{fs}=\mathrm{vols} / \mathrm{vol0}$

Beta $=\mathrm{fs} /(1-\mathrm{fs})$;

parmA $=0.134$

parmB $=0.03$;

$\mathrm{R}=1.987$;

$\mathrm{kp} 0=2.95 \mathrm{e} 7 * \exp \left(-4353 /\left(\mathrm{R}^{*} \mathrm{~T}\right)\right)$;

$\mathrm{kt} 0=5.88 \mathrm{e} 9 * \exp \left(-701 /\left(\mathrm{R}^{*} \mathrm{~T}\right)\right) ; \quad \mathrm{kd}=6.32 \mathrm{e} 16 * \exp (-15.43 \mathrm{e} 3 / \mathrm{T}) ;$

thetat $=2.33 \mathrm{e} 3$;

thetap $=3500$;

$\mathrm{F}=0.58$;

Cinit $0=0.01548$; 
$\mathrm{t} 0=0 ; \mathrm{tmax}=5$;

$\mathrm{Al}=(\operatorname{eye}(\mathrm{N}+1)-\operatorname{diag}($ ones $(\mathrm{N}, 1), 1)) ;$

$\operatorname{Al}(1,1)=0$

$\%$ Initial Values for the ODE Integration

y0 = $[\operatorname{zeros}(\mathrm{N}+1,1) ; \operatorname{zeros}(2 * \mathrm{~N}+1,1) ;$ Cinit $0 ; 0]$;

cpt=cputime;

options=odeset('RelTol', 1e-6, 'Abstol', 1e-12, 'InitialStep', 1e-6);

$[\mathrm{t}, \mathrm{y}]=\mathrm{ode} 15 \mathrm{~s}($ 'mymodel',[t0 tmax $], \mathrm{y} 0$,options);

$\%$ ODE $15 \mathrm{~s}$ is an integration algorithm for systems of stiff differential equations

elapt=cputime-cpt

$\mathrm{P} \quad=\mathrm{y}(:, 1: \mathrm{N}+1)$;

D = y(:,N+2:3*N+2);

Cinit $=y(:, 3 * N+3)$

convr $\quad=y(:, 3 * N+4)$;

$\%$ Vector generation for the computation of molecular weights

$10=$ ones $(\mathrm{N}+1,1)$;

$11=[1: \mathrm{N}+1]^{\prime}$;

$12=11 .^{\wedge} 2$;

$\mathrm{m} 0=\operatorname{ones}(2 * \mathrm{~N}+1,1)$

$\mathrm{ml}=[1: 2 * \mathrm{~N}+1]^{\prime}$

$\mathrm{m} 2 \cdot=\mathrm{m} 1 \cdot^{\wedge} 2$

lambda $0=\mathrm{P} * 10$; 


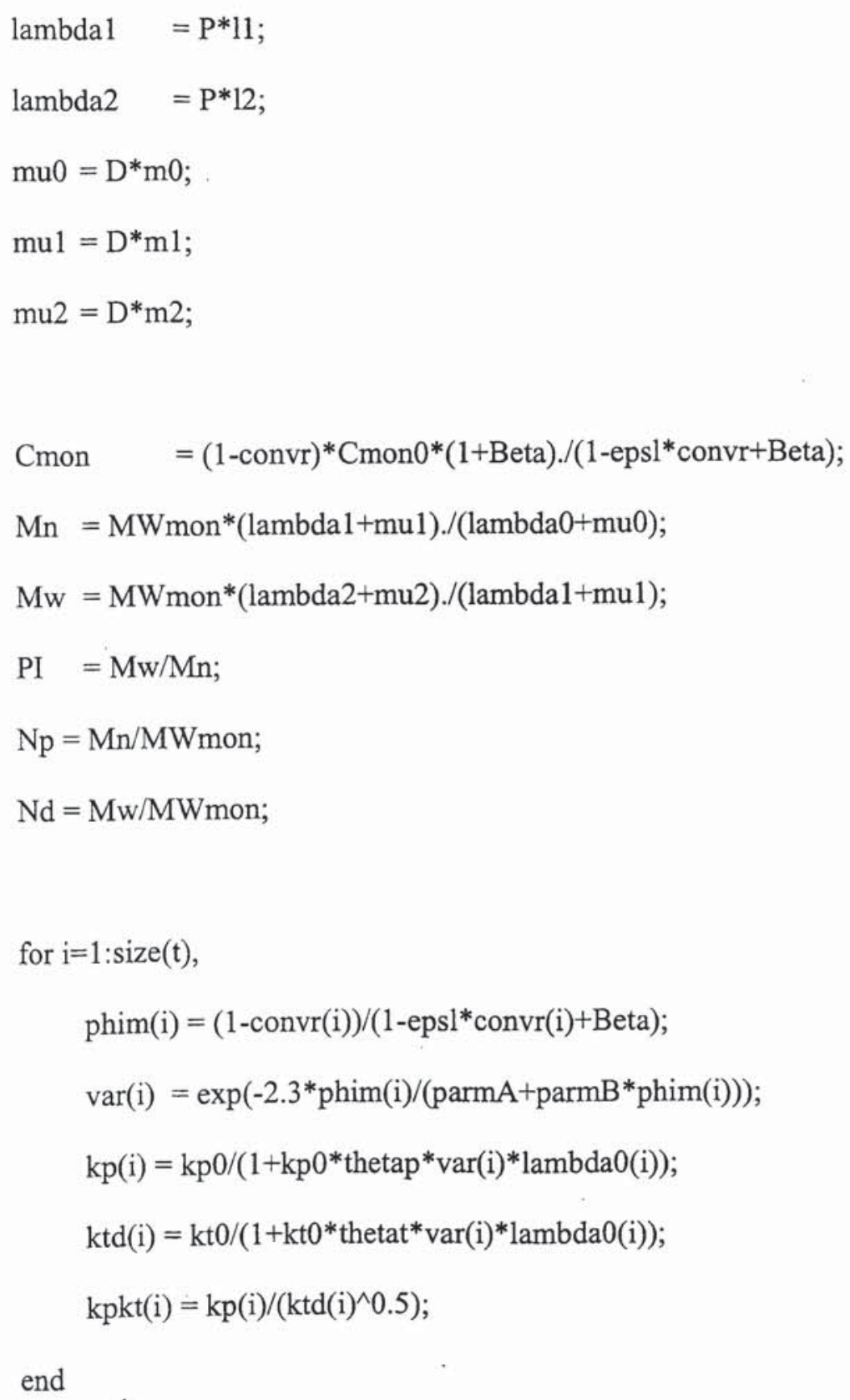


Appendix I: MATLAB Code for FRP Simulations

\%save 300_50_1548.mat

Subroutine for ODE generation:

function ydot=samp $(\mathrm{t}, \mathrm{y})$

global N T Cmon0 vol0 epsl Beta parmA parmB R kp0 kt0 kd thetat thetap F Cinit0 A1

$$
\begin{aligned}
& P \quad=y(1: N+1) ; \quad D \quad=y(N+2: 3 * N+2) ; \\
& \text { Cinit }=y(3 * N+3) ; \quad \text { convr }=y(3 * N+4) ; \\
& \text { Pstar }=[1 ; y(1: N)] ; \quad Q \quad=[y(1: N+1) ; z \operatorname{ros}(N, 1)] ; \\
& \text { Cmon }=(1-\text { convr })^{*} \text { Cmon } 0 *(1+\text { Beta }) /(1-\text { eps } 1 * \operatorname{convr}+\text { Beta }) ;
\end{aligned}
$$

$$
\begin{aligned}
& \text { phim }=(1-\text { convr }) /\left(1-\text { eps } 1^{*} \text { convr }\right) ; \\
& \text { var }=\exp (-2.3 * \text { phim } /(\text { parmA }+ \text { parmB } * \text { phim })) \\
& \mathrm{kp} \quad=\mathrm{kp} 0 /(1+\mathrm{kp} 0 * \text { thetap*var*sum }(\mathrm{P})) ; \\
& \mathrm{ktd}=\mathrm{kt} 0 /(1+\mathrm{kt} 0 * \text { thetat*var*sum }(\mathrm{P})) ; \\
& \mathrm{ktc}=0.135 * \mathrm{ktd} ;
\end{aligned}
$$$$
\mathrm{C} \quad=[]
$$

for $\mathrm{i}=0: \mathrm{N}$

$$
\mathrm{C} \quad=\operatorname{rcdiag}(\mathrm{C}, \operatorname{diagonal}(\operatorname{zeros}(\mathrm{i}, \mathrm{i}), \text { eye }(\mathrm{N}+1-\mathrm{i})) * \mathrm{P}) ;
$$

end

$\mathrm{A}=\mathrm{kp} *$ Cmon*A1+diagonal $(2 * \mathrm{~F} * \mathrm{kd} * \operatorname{Cinit}, \mathrm{zeros}(\mathrm{N}))$;

grp $=$ epsl*kp*(1-convr $) /(1$-epsl*convr+Beta); 
grp $1=$ vol $0 *(1-$ eps $1 *$ convr + Beta $)$

ydot $1=A^{*}$ Pstar $+($ grp-ktd-ktc)*sum $(\mathrm{P}) * \mathrm{P}$;

\%ydot $2=\mathrm{ktd} * \operatorname{sum}(\mathrm{P}) * \mathrm{Q}+\operatorname{sum}(\mathrm{P}) * \operatorname{grp} * \mathrm{D}$;

$y \operatorname{dot} 2=\mathrm{ktd} * \operatorname{sum}(\mathrm{P}) * \mathrm{Q}+\mathrm{ktc} * \mathrm{C} * \mathrm{P}+\operatorname{sum}(\mathrm{P}) * \operatorname{grp} * \mathrm{D}$;

$\%$ ydot3 $=(\operatorname{grp} * \operatorname{sum}(\mathrm{P})-\mathrm{kd}) *$ Cinit;

$y \operatorname{dot} 3=(\operatorname{grp} * \operatorname{sum}(\mathrm{P})-\mathrm{kd}) *$ Cinit;

$\mathrm{ydot} 4=\mathrm{kp} *(1-$ convr $) * \operatorname{sum}(\mathrm{P})$;

ydot=[ydot $1 ; y d o t 2 ; y d o t 3 ; y d o t 4] ;$ 


\section{Appendix II}

\section{The Advanced Photon Source: A Primer}

The Advanced Photon Source (APS) is a national synchrotron radiation light source research facility. It is a third-generation synchrotron source used for the production of high-flux, high-brilliance $\mathrm{x}$-rays. Third generation storage rings maximize the $\mathrm{x}$-ray beam qualities such as flux and brilliance.

$\mathrm{X}$-rays are produced with the help of a series of 5 major components: (A) Linear Accelerator (LINAC), (B) Accumulator Ring, (C) Booster/synchrotron, (D) Storage Ring(SR), and (E) Beamlines.

The beam acceleration and the storage process begins at the electron gun in the linear accelerator, which emits electrons with $100 \mathrm{keV}$ of energy. Electrons from the LINAC are then accelerated to energy of $450 \mathrm{Mev}$ by a series of accelerating structures. The APS positron accumulator ring (PAR) is a $450-\mathrm{MeV}$ positron storage ring (SR) that accumulates positrons from the linear accelerator for injection into the synchrotron. 
Later the beam is injected to the booster which increases its energy to $7 \mathrm{Gev}$. Here the accelerating force is supplied by electrical fields from what are called as Radio Frequency (RF) cavities.

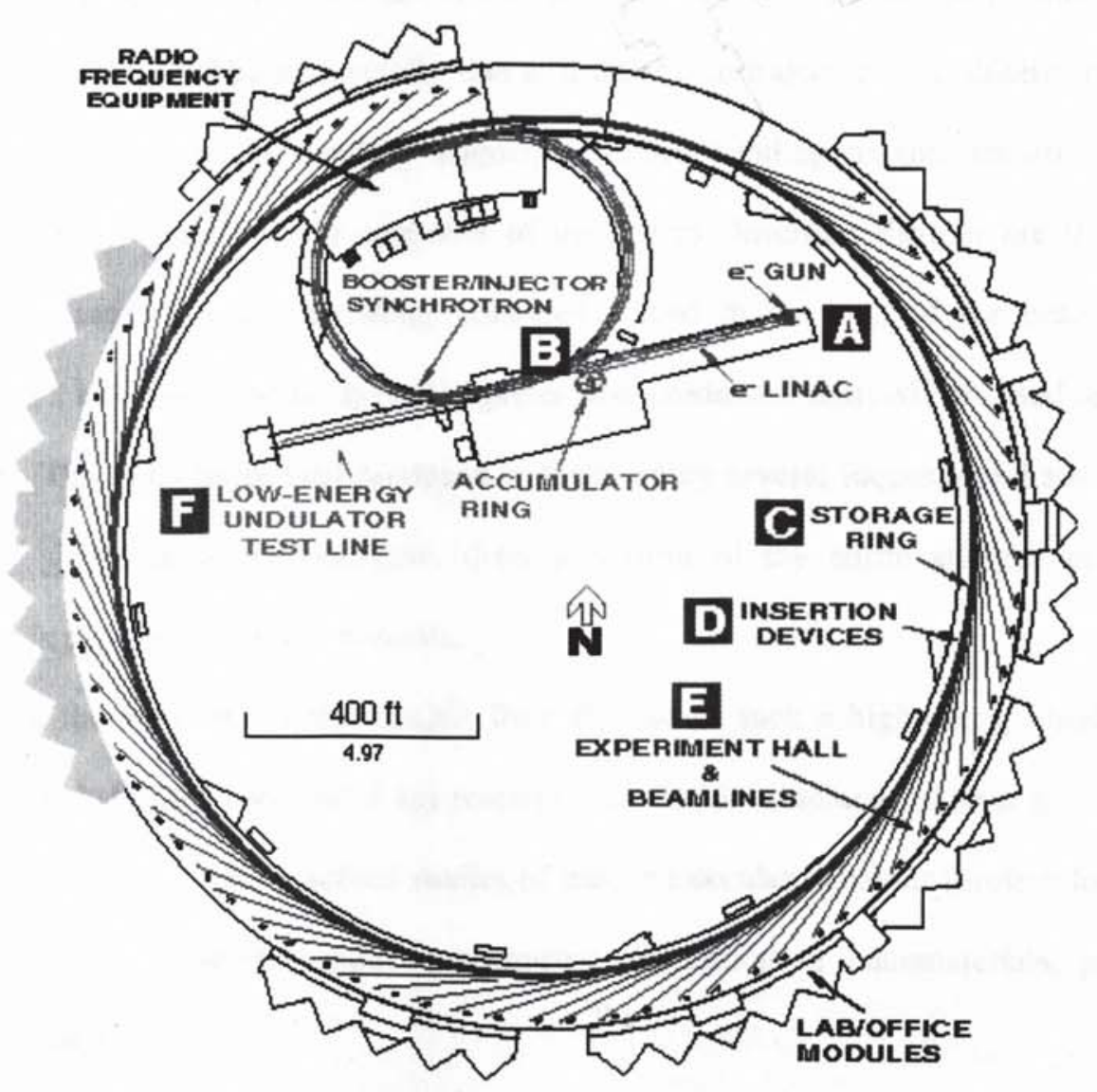

Figure II.1: Schematic of the Advanced Photon Source.

From the booster the electrons are then injected into the storage ring located inside the experimental hall. APS has 40 sectors: 5 of which are equipped with injection and RF equipment while the other 35 are used to provide insertion device and bending magnet radiation. Each of the 35 sectors has 2 beamlines: one originating at a bending magnet in 


\section{Appendix II: A Primer on APS}

the SR lattice and the other at an insertion device. The beam of electrons is steered and focused by 1097 powerful electromagnets. Electrons in the storage ring loose their energy by emitting the synchrotron radiation, and decelerate at every turn, at the rate of about $6 \mathrm{MeV}$. The loss of energy is continuously replenished by the $352 \mathrm{MHz}$ storage ring RF system.

Bending magnets and insertion devices are the major components for the production of $\mathrm{x}$-rays. Radiation emitted tangentially due to a change in trajectory and deceleration of electrons imposed by the bending magnets is called bending magnet radiation. The insertion devices used mainly comprise of undulators. Insertion devices are the N-S permanent magnets with alternating polarities placed in the path of the beam. The undulators have lower fields, spaced together and produce a narrowly-focused spot of radiation. The $\mathrm{x}$-ray beam thus produced is absorbed by several focusing and absorbing elements placed in every beamline. Only a portion of the entire energy spectrum available is used for scientific research.

Some of the applications that benefit from the use of such a high-energy, high flux radiation include (but not limited to) research into the development of next generation synchrotron sources, time-resolved studies of macromolecular behavior (protein folding, reaction kinetics), radiation-induced chemistry, diffraction of nanomaterials, protein crystallography, etc.

More information on the Advanced Photon Source is available at http://www.aps.anl.gov. 


\section{Appendix III}

\section{Beamline Configuration: 2-BM}

Beamline 2-BM-B of the Advanced Photon Source (APS) was first commissioned as a bending magnet beamline for the studies of synchrotron radiation instrumentation and science. Apart from the instrument studies, this beamline was also configured for deep x-ray lithography exposures in 1996 by Dr. Barry Lai and his colleagues. Since its commissioning, the beamline was also modified to perform microtomography, combinatorial characterization of materials and x-ray diffraction microscopy. As shown in Figure III, 2-BM has two stations (hutches): 2-BM-A and 2-BM-B.

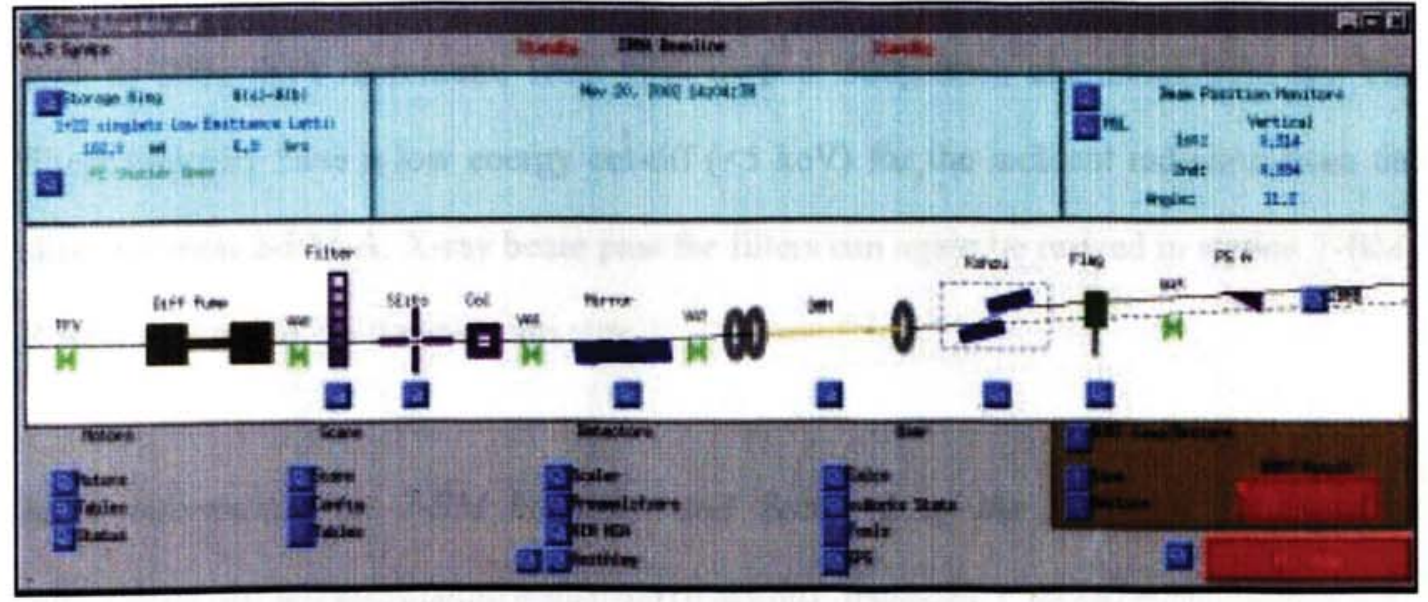




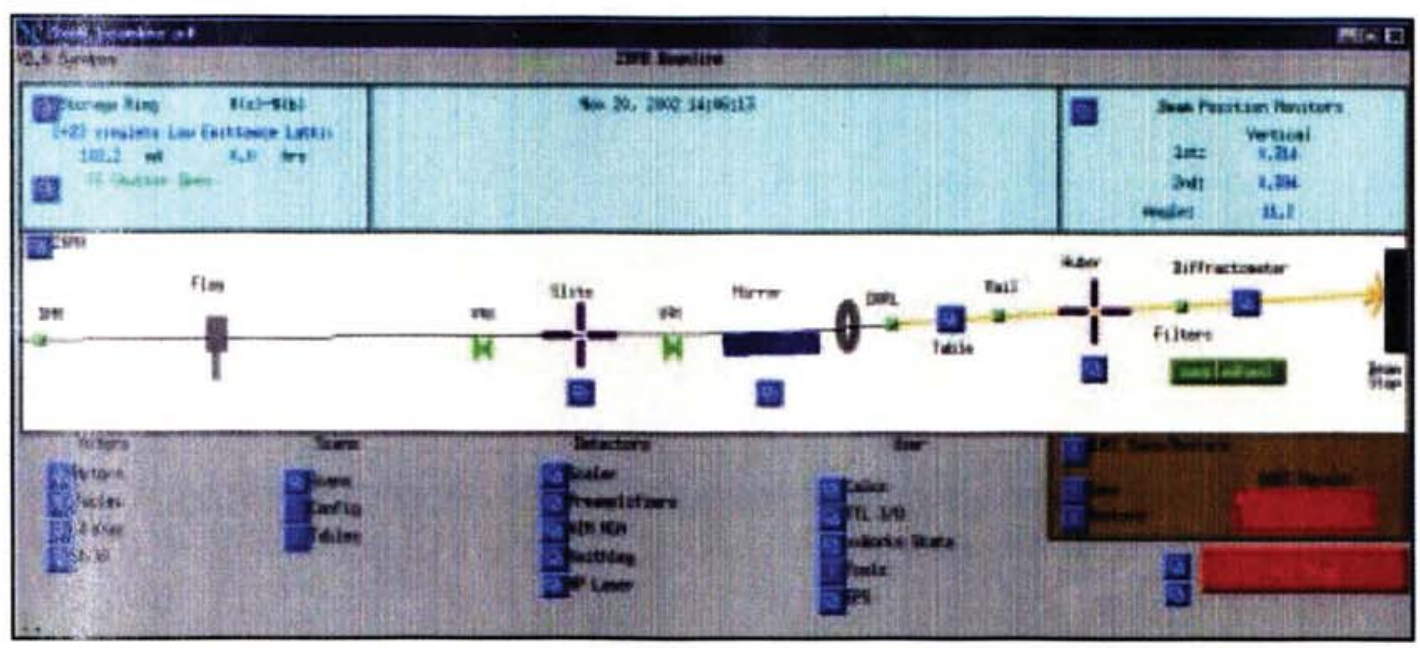

Energy spectrum of the incident radiation in 2-BM-B is varied by inserting either a dynamic multilayered monochromator (DMM) or a double crystal monochromator (DCM) in station 2-BM-A. DMM is used when soft $\mathrm{x}$-rays $(0.5-4 \mathrm{keV})$ are required, while DCM is used for the regular hard x-ray exposures in a "pink beam" mode. The radiation transmitted through either of the DMM or DCM is then reflected off a mirror coated with either chromium or platinum and held at an angle of incidence of $0.15^{\circ}$, which attenuates the photons with energy higher than 20 and $35 \mathrm{keV}$ respectively. $\mathrm{X}$-rays reflected off the mirror were then sent through a slit in 2-BM-B that is $100 \mathrm{~mm}$ wide and $7 \mathrm{~mm}$ tall. The slit size limited beam is then sent through one of the eight available filters such as $300 \mu$-thick aluminum, $1 \mathrm{~mm}$-thick carbon, $500 \mu$-thick aluminum foils etc. The filters typically have a low energy cut-off $(<5 \mathrm{keV})$ for the incident radiation from the slits in station 2-BM-A. X-ray beam past the filters can again be resized in station 2-BMB with the help of the downstream slits.

More information on 2-BM beamline and Sector 2 of the APS can be found at http://beam.aps.anl.gov/pls/apsweb/sri0010. display frameset? $i$ menuI $=25$. 